\title{
Linearity Improvement In Vernier \\ Delay Chain Based Time Difference Amplifiers
}

\author{
by
}

Niranjan Bangalore Ramesh

\author{
A thesis submitted to the Faculty of Graduate and \\ Postdoctoral Affairs in partial fulfillment of the \\ requirements for the degree of
}

Masters Of Applied Science in

Electrical and Computer Engineering

Carleton University

Ottawa, Ontario

(C)2014

Niranjan Bangalore Ramesh 


\section{Abstract}

Time difference amplifiers are used in fluorescence experiments where the input time differences are in picoseconds. The key challenge is to provide the linearity of such amplifiers with sufficient gain over the required input range. This amplifier

has a gain greater than $2 \frac{\mathrm{p} s}{\mathrm{p} s}$, with the input ranging from $10 \mathrm{pS}$ to $1000 \mathrm{pS}$. The gain error is found to be less than $10 \%$ at a temperature of $25^{\circ} \mathrm{C}$, voltage range of $800 \mathrm{mV}$ to $1.2 \mathrm{~V}$. The overall power consumption from simulation is $4 \mathrm{~mW}$.

This design uses differential signalling based architecture and is an open loop system to realize these specifications. Initially results of the design were verified using simulation techniques and then the physical implementation was done resulting in an integrated circuit that was fabricated and tested. While the open loop approach in this implementation will make it unable to correct the gain with temperature variations, the circuit has features that can integrate well with a feedback system to convert it to a closed loop system. 


\section{Acknowledgement}

This thesis has been a very intense and tough journey for me and a very important mile stone in my career. There are several who have stood by me and helped me by providing the required support in several ways. I wish to thank them personally here. The Department of Electronics, Carleton University, Carleton, Ottawa for allowing me to carry out this research and its excellent infrastructure. My professor, Dr.Leonard MacEachern, who graciously allowed me to carry out this research under his expert guidance. His inspiration, guidance and advice have been very timely, invaluable and has been the key factor that led to the completion of this thesis. My heart felt thanks to him. My sincere thanks to Dr. Christopher Salthouse University of Massachusetts, Amherst, for giving me this opportunity to do collaborative research and enabling me to work on latest concepts that are in the early research stage. I thank him for his time and guidance. Mr.Nagui Mikhail and Mr. Rob Vandusen, here at the Carleton University have extended a lot of technical help time and again, not to forget advice and support. I also wish to thank my parents, Mr.Ramesh Iyer and Mrs. Bhuvana Ramesh, Bangalore, India who have provided me the required support all the way through this course and especially during this thesis.

"My favourite programming language is SOLDER" - Bob Pease. 


\title{
List Of Acronyms
}

\author{
ESD : Electrostatic Discharge \\ SMA : SubMiniature Version A \\ DUT : Device Under Test \\ IC : Integrated Circuit \\ PCB : Printed Circuit Board \\ TDA : Time Difference Amplifier \\ A/D : Analog to Digital \\ TDC : Time to Digital Converter \\ DTC : Digital to Time Converter \\ INL : Integral Non Linearity \\ DNL : Differential Non Linearity \\ DLL : Delay Locked Loop \\ CCD : Charge Coupled Device \\ LASER : Light Amplification By Stimulated Emission of Radiation \\ LIDAR : Laser Imaging Detection and ranging \\ DDS : Direct Digital Synthesis \\ NCO : Numerically Controlled Oscillator \\ MOSFET : Metal Oxide Semiconductor Field Effect Transistor
}


CML : Current Mode Logic

DC : Direct Current

SOT : Small Outline Transistor

LQFP : Low Profile Quad Flat Package

SOIC : Small Outline Integrated Circuit

TSSOP : Thin Shrink Small Outline Package

DIP : Dual Inline Plastic Package

RoHS : Reduction Of Hazardous Substances

DSO : Digital Storage Oscilloscope

DLL : Delay Locked loop

PVT : Process Voltage Temperature

MUTEX : Mutually Exclusive 


\section{Contents}

$\begin{array}{llr}1 & \text { Introduction and motivation } & 15\end{array}$

1.1 Thesis Outline . . . . . . . . . . . . . . . . . 16

$\begin{array}{lll}2 & \text { Time Difference Circuits and applications } & 17\end{array}$

2.1 Introduction to Time Difference Circuits . . . . . . . . . . . . 17

2.1.1 Time difference Arbiter . . . . . . . . . . . . . . . . . . . 18

2.1.2 Time Difference Adder . . . . . . . . . . . . . . . . . . . . 22

2.1.3 Time to digital Convertors . . . . . . . . . . . . . 27

2.1.4 Digital to time converter . . . . . . . . . . . . . 30

2.2 Applications of Time Difference Circuits . . . . . . . . . . . . . . 34

$2.2 .1 \quad$ LIDAR . . . . . . . . . . . . . . . . . . 34

2.2 .2 Fluorescence. . . . . . . . . . . . . . . . . . . . 35

2.2.3 Timing analysis for high speed circuits . . . . . . . . . . 36

2.2.4 Direct Digital Synthesis _ . . . . . . . . . . . . . 36

2.2.5 Particle Research for photon Counting . . . . . . . . . . . 37

3 Time Difference Amplifiers $\quad 38$

3.1 Architecture Overview . . . . . . . . . . . . . . . . . 39

3.1.1 Switched capacitor based time difference amplifier . . . . . . 40 
3.1.2 Mutex Based Time Difference Amplifier . . . . . . . . . . . 42

3.1.3 Inverter delay chain architecture . . . . . . . . . . . . . . 46

3.1.4 Delay Cell Architectures . . . . . . . . . . . . . . . . . . . . 48

3.1.5 Current Starved Inverter Design . . . . . . . . . . . . . 49

3.1.6 Other Architectures for Delay Cell . . . . . . . . . . . . 51

3.1.7 Time Difference Amplifier using Differential signalling . . . . 55

3.1.8 Variable delay cell using differential logic . . . . . . . . . 57

$\begin{array}{lll}4 & \text { Implementation } & 60\end{array}$

4.1 Design Specifications . . . . . . . . . . . . . . 60 60

4.2 Switched Inverter chain based approach . . . . . . . . . . . . 61

4.3 Differential signalling based TDA . . . . . . . . . . . . 66

4.3.1 Architecture . . . . . . . . . . . . . 66

4.3.2 Inverter Design . . . . . . . . . . . . . . . . . . . 69

4.3.3 Multiplexer design . . . . . . . . . . . . . . 73

4.3.4 Delay cell Implementation . . . . . . . . . . . . . 77

4.3.5 Chip Level Implementation _. . . . . . . . . . . . . 80

4.4 Layout Implementation . . . . . . . . . . . . . . . . . . 82

4.5 Simulation Results . . . . . . . . . . . . . . . 85

5 Testing methodology and Design $\quad 93$

5.1 Test Board Circuit Design . . . . . . . . . . . . . . . 93

5.2 Printed Circuit Board Design _. . . . . . . . . . . . 96

$5.2 .1 \quad$ PCB Flow . . . . . . . . . . . . . . . . . . . . . 96

5.2 .2 PCB Specifications . . . . . . . . . . . . . . . . 97 
5.3 PCB design details . . . . . . . . . . . . . . . . . . . . . . . 98

5.4 Printed Circuit Board Bring up Plan . . . . . . . . . . . . . . 100

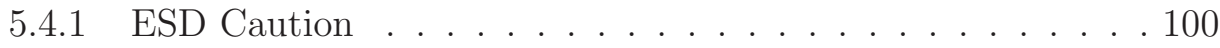

5.4.2 Equipments required from board bring up . . . . . . . . 100

5.4 .3 Cold Resistance Test . . . . . . . . . . . . . . . . . . 102

5.4 .4 Power Supply Test . . . . . . . . . . . . . . . . 103

5.4 .5 Power Sequencing . . . . . . . . . . . . . . . . . 104

5.4 .6 Delay line IC Calibration . . . . . . . . . . . . . . 105

5.4 .7 Silicon Bring Up . . . . . . . . . . . . . . . . . . 106

5.5 Time Difference Amplifier Linearity Test . . . . . . . . . . . . . . 107

$6 \quad$ TDA Testing and Debug 109

6.1 Test Bench Setup . . . . . . . . . . . . . . . 111

6.2 Analysis of Failed DUT . . . . . . . . . . . . . . . . . . 114

7 Conclusion and future work 122

Appendix A Tools Used and Design Methodology 132

$\begin{array}{lll}\text { Appendix B } & \text { Test Board Schematics } & 134\end{array}$

$\begin{array}{ll}\text { Appendix C Bill of Materials } & 137\end{array}$

$\begin{array}{ll}\text { Appendix D PCB Gerber } & 138\end{array}$ 


\section{List of Figures}

2.1 Arbiter . . . . . . . . . . . . . . . . . . . 18

2.2 S R Latch based arbiter . . . . . . . . . . . . . . . . . . 19

2.3 Metastability Region . . . . . . . . . . . . . . . . . . . 19

2.4 Arbiter with Metastability filter . . . . . . . . . . 20

2.5 Modified Arbiter Circuit . . . . . . . . . . . . . . . . 21

2.6 Time Difference Adder . . . . . . . . . . . . . . . . . . . . . . 22

2.7 Time Difference adder implementation . . . . . . . . . . . . 23

2.8 Delay tuner . . . . . . . . . . . . . . . . . . . . . . . . . . . 24

2.9 Time Difference adder based on inverter chain . . . . . . . . . . 25

2.10 Time Difference adder sample plot . . . . . . . . . . . . 26

2.11 Basic TDC architecture . . . . . . . . . . . . . 27

2.12 Basic TDC clock scheme . . . . . . . . . . . . . . . . . 28

2.13 Basic TDC output waveforms . . . . . . . . . . . . 28

2.14 Flash TDC . . . . . . . . . . . . . . . . . . . . 29

2.15 Basic Digital to Time converter . . . . . . . . . . . . . . 30

2.16 DTC delay cell . . . . . . . . . . . . . . . . . . 31

2.17 DTC with closed loop feedback . . . . . . . . . . . . . . 32

2.18 Output transfer function of DTC . . . . . . . . . . 32 
2.19 DNL plot for DTC . . . . . . . . . . . . . . . 33

2.20 DNL plot for DTC . . . . . . . . . . . . . . . . 33

2.21 LIDAR system using Time difference Circuits . . . . . . . . . . . 34

2.22 Florescence basic architecture . . . . . . . . . . . . . . . 35

2.23 On chip timing correction . . . . . . . . . . . . . 36

2.24 Basic Photon Counting System . . . . . . . . . . . . . . 37

3.1 Time Difference Amplifier . . . . . . . . . . . . . . . . . . . 38

3.2 Switched capacitor based Time difference Amplifier . . . . . . . . . 40

3.3 Switched capacitor based Time difference Amplifier . . . . . . . . . 41

3.4 Mutex Based Time Difference Amplifier . . . . . . . . . . . . . . . 43

3.5 Mutex Based Time Difference Amplifier response . . . . . . . . . . 43

3.6 MUTEX with Metastability filter . . . . . . . . . . . . . . 44

3.7 Gated mutex circuit f . . . . . . . . . . . . . . . 46

3.8 Variable inverter delay chain based amplifier . . . . . . . . . . . . 47

3.9 Switching Delay Cell . . . . . . . . . . . . . . . . . . 47

3.10 Inverter delay Cell . . . . . . . . . . . . . . . . . . . . . . 48

3.11 Current staving Inverter delay Cell . . . . . . . . . . . . . 50

3.12 Dual Inverter based delay cell . . . . . . . . . . . . . . . . 51

3.13 Modified Current Mirror . . . . . . . . . . . . . . . . . 52

3.14 TDA block diagram with tuning bias . . . . . . . . . . . 52

3.15 Variable Delay cell using varactor . . . . . . . . . . . . . . 53

3.16 Selectable Output Vernier Chain delay . . . . . . . . . . . . 54

3.17 Differential input type TDA . . . . . . . . . . . . . 55

3.18 Time Amplifier . . . . . . . . . . . . . . . . . . 56 
3.19 Differential Type Delay Cell . . . . . . . . . . . . . . . . 57

3.20 Time difference amplifier with differential delay cell . . . . . . . . 58

4.1 TDA chain with single ended input Inverters Hierarchical Symbol . 61

4.2 Single ended signalling based delay cell . . . . . . . . . . . . . 62

4.3 TDA chain with single ended input Inverters . . . . . . . . . 63

4.4 TDA chain with single ended input inverters transfer function and

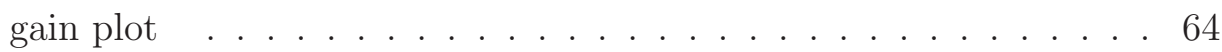

4.5 TDA chain with single ended input Inverters transfer function . . . 65

4.6 Basic Architecture : Delay Cell . . . . . . . . . . . . . . . 66

4.7 Basic Architecture : TDA . . . . . . . . . . . 67

4.8 Buffer Block Diagram . . . . . . . . . . . . . . . . . . . . 68

4.9 Inverter Hierarchical symbol . . . . . . . . . . . . . . . . . 69

4.10 Inverter Schematics . . . . . . . . . . . . . . . . 70

4.11 Inverter Schematics . . . . . . . . . . . . . . . . 71

4.12 Inverter Layout . . . . . . . . . . . . . . . . . . . 72

4.13 2:1 Multiplexer Symbol . . . . . . . . . . . . . . . . 73

$4.142: 1$ Multiplexer Schematic . . . . . . . . . . . . . . 74

4.15 2:1 Multiplexer Schematic Detail . . . . . . . . . . . . . 75

$4.162: 1$ Multiplexer Layout . . . . . . . . . . . . . . . . . 75

4.17 Delay Cell Implementation . . . . . . . . . . . . . . . . . . . 77

4.18 Delay Cell Implementation Symbol _ . . . . . . . . . . . . . 78

4.19 Delay Cell Implementation Layout _ . . . . . . . . . . . . 79

4.20 Time difference amplifier system implementation . . . . . . . . . . 80

4.21 TDA layout . . . . . . . . . . . . . . . . . . . . . . . . 82 
4.22 Full Chip layout . . . . . . . . . . . . . . . . . . . 84

4.23 Simulation Test Bench . . . . . . . . . . . . . . . 85

4.24 TDA output waveform . . . . . . . . . . . . . 86

4.25 TDA transfer characterestics, gain plot . . . . . . . . . . . 88

4.26 TDA transfer characterestics, output delay . . . . . . . . . . . . 89

4.27 Gain Curve . . . . . . . . . . . . . . . . . . . 9 90

4.28 Gain Curve at Fast Corner . . . . . . . . . . . . . . . . . . . 91

4.29 Short Delay characterization . . . . . . . . . . . . . . . 92

4.30 Long Delay characterization . . . . . . . . . . . . . . . 92

5.1 TDA Test Board Architecture . . . . . . . . . . . . . 94

5.2 Board Stack Up . . . . . . . . . . . . . . . . . . . . 97

5.3 PCB Layout without routing . . . . . . . . . . . . . . . . 98

5.4 PCB Routing . . . . . . . . . . . . . . . . . . . . . . . . 99

5.5 PCB bring up flow . . . . . . . . . . . . . . . . 101

5.6 PCB Power Rails . . . . . . . . . . . . . . . . . . . . 102

5.7 PCB Power Rails Test . . . . . . . . . . . . . . . . 103

5.8 PCB Power Rails Test . . . . . . . . . . . . . . . 105

5.9 DUT . . . . . . . . . . . . . . . . . . . . 106

5.10 Linearity test . . . . . . . . . . . . . . . 108

6.1 Lab Test Bench . . . . . . . . . . . . . . . . . . . . . 110

6.2 Test Bench Setup . . . . . . . . . . . . . . . . . . . . 111

6.3 Input Waveform Applied to DUT . . . . . . . . . . . . . . . . 112

6.4 Time Difference Signal . . . . . . . . . . . . . . . . . . . . 113

6.5 Probing Station Test Bench Setup . . . . . . . . . . . . . . 118 
6.6 Probes . . . . . . . . . . . . . . . . . . . . . 118

6.7 Die prepared for probing . . . . . . . . . . . . . . 119

6.8 Die with probes . . . . . . . . . . . . . . . . 119

6.9 Die with probes . . . . . . . . . . . . . . . 121

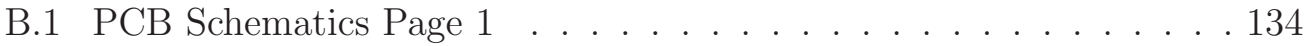

B.2 PCB Schematics Page $2 \ldots \ldots . \ldots . \ldots . \ldots . \ldots . \ldots 135$

B.3 PCB Schematics Page $3 \ldots \ldots 136$

D.1 Gerber : Top Layer . . . . . . . . . . . . . . . . . . . . . 138

D.2 Gerber : Bottom Layer . . . . . . . . . . . . . . . . . 139

D.3 Gerber : Ground Plane Layer . . . . . . . . . . . . . . . . 139

D.4 Gerber : Power Plane Layer . . . . . . . . . . . . . . . . . 140

D.5 Gerber : Solder mask top Layer . . . . . . . . . . . . . . . . 140

D.6 Gerber : Solder mask Bottom Layer . . . . . . . . . . . . . . 141

D.7 Gerber : Silkscreen top Layer . . . . . . . . . . . . . . . 141 


\section{List of Tables}

2.1 Arbiter . . . . . . . . . . . . . . . . . . . 18

3.1 S R Flip Flop Truth Table . . . . . . . . . . . . . . . . . . . . 42

3.2 Result Comparision Summary . . . . . . . . . . . . . . . . 59

4.1 Inverter Pin descriptions . . . . . . . . . . . . . . . . . . 69

4.2 Inverter design Transistor Aspect Ratios . . . . . . . . . . . . . 72

$4.32: 1$ Mux Pin descriptions . . . . . . . . . . . . . . 73

$4.4 \quad 2: 1$ Multiplexer truth table . . . . . . . . . . . . . 74

4.5 2:1 Multiplexer design Transistor Aspect Ratios . . . . . . . . . 76

4.6 Delay cell pin descriptions . . . . . . . . . . . . . . . 78

4.7 TDA Chip Pin descriptions . . . . . . . . . . . . . 81

5.1 XOR Gate truth table . . . . . . . . . . . . . . . . . 95

5.2 PCB Specifications . . . . . . . . . . . . . . . . . . . . 97

5.3 Test flow . . . . . . . . . . . . . . . . . . . . 101

5.4 Cold Resistance Test Result . . . . . . . . . . . . . . . . . . . . 102

5.5 Voltage Rail Test Results . . . . . . . . . . . . . . . . . 103

5.6 Cold Resistance Test Result after DUT Populated . . . . . . . . . . 106 
6.1 Board 1 power up tests with DUT populated . . . . . . . . . . . 114

6.2 Board 1 static input tests with DUT populated . . . . . . . . 115

6.3 DUT3 power up tests with DUT populated . . . . . . . . . . 115

6.4 DUT 3 test result . . . . . . . . . . . . . . . . 116

6.5 DUT 4 test result . . . . . . . . . . . . . . . . . 117

6.6 Test circuit without ESD protection results . . . . . . . . . . . 120

6.7 Test circuit without ESD protection results . . . . . . . . . . . 120

A.1 Tools Used . . . . . . . . . . . . . . . . . . . . . . . 132

C.1 Bill Of Materials . . . . . . . . . . . . . . . 137 


\section{Chapter 1}

\section{Introduction and motivation}

The main challenges in the florescence is the expensive set-up of lasers and photodetectors that capture events that occur in one trillionth of a second. Processing the signals is a challenge. It becomes difficult for the system to measure the time difference between the signals, as they occur in pico seconds.

This circuit is a part of a bigger experiment setup for florescence which are used for cytology, and analysis of of chemical properties of cells.

The nature of the experiment is analysis of the signals whose time difference are in pico second intervals. The light energy is converted into electrical signals which can be processed to analyse the cell structures and their chemical properties.

As voltage amplifiers were designed to amplify weak signals and to provide gain, a time difference amplifier does a similar task of amplifying the time difference between two signals. The main challenge in this time difference amplifier is the linearity and gain. 


\subsection{Thesis Outline}

This thesis shows highlights of different time difference circuits and its applications and also focusing on the linearity issue in vernier chain based TDA circuits. The document also gives the idea of the new architecture for improving linearity. Simulation and test results are also mentioned along with testing results.

Chapter 1 gives a general introduction to the topic and the research methodology used. Chapter 2 gives a bird's eye view of the different time difference circuits, their architectures and the applications.

Chapter 3 gives a detailed literature survey focusing on only Time Difference Amplifier circuits. Different architectures are compared their drawbacks and results compared with the new architecture proposed in this thesis. A detailed comparison is made and tabulated results are shared.

Chapter 4 explains the specifications and the issues with vernier chain amplifier and the end application. The architecture and circuit level implementations are discussed, and the simulation results are shown.

Chapter 5 shows the testing methodology and the PCB design. In this chapter the test setup and flow are explained. The test results of the experiments are also discussed and concludes the thesis. 


\section{Chapter 2}

\section{Time Difference Circuits and}

\section{applications}

This chapter describes the background on time difference circuits, highlighting the application that is related to the work done in the area of Time Difference Amplifiers.

\subsection{Introduction to Time Difference Circuits}

Time difference circuits are used where the signals have very small time differences or delay between them. The classification of the time difference circuits are based on the application and are discussed briefly in this chapter.

- Time difference Arbiter

- Time difference Adders

- Time difference Amplifier

- Time to Digital converters

- Digital to time converters 


\subsubsection{Time difference Arbiter}

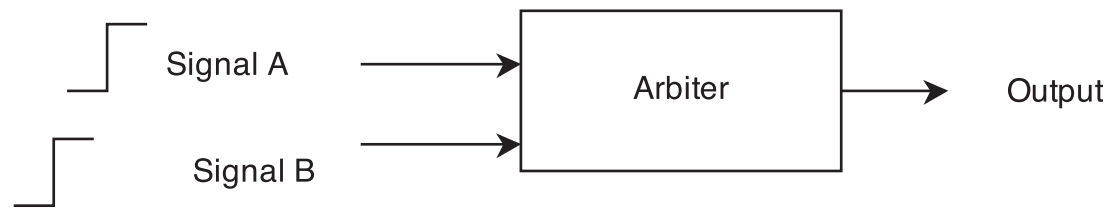

Figure 2.1: A time difference arbiter is similar to a voltage comparator that compares two voltage inputs and provides an output that is the result of such a comparison. A time difference arbiter does exactly the same operation but in the time domain. It compares two signals in time and provides an output that is a result of such a comparison.

If two signals $\mathrm{A}$ and $\mathrm{B}$ are compared an arbiter circuit the output simply tells us which signal occurred first. In the circuit shown in figure 2.1 , say Signal A arrived first and Signal B later, the output of the arbiter will be a logic '1', else a ' 0 '. The concept is shown in table 2.1 .

\begin{tabular}{|c|c|c|}
\hline Signal Arrives First & Signal Arrives Next & Output \\
\hline Signal A & Signal B & 1 \\
\hline Signal B & Signal A & 0 \\
\hline
\end{tabular}

Table 2.1: Truth table illustrating the working of the tie difference arbiter. 


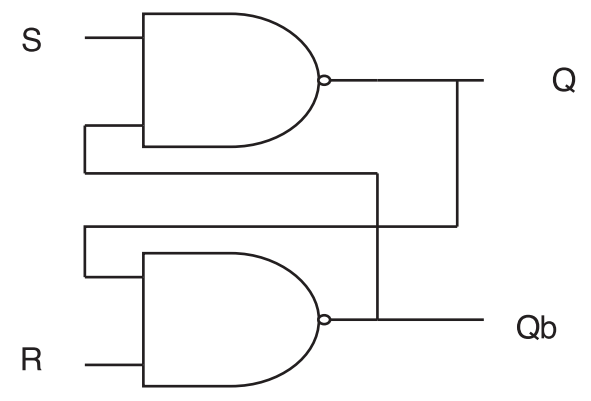

Figure 2.2: S R Latch realised using NAND gates. The S R latch is formed by the feedback between the output and the inputs [1]

Figure 2.2 shows a simple arbiter is realized using a S R Latch. S R latches in normal practice are used with either one of the inputs high or both inputs low, but never with both inputs high. This state when both its inputs are high is termed the forbidden or metastable state shown below.

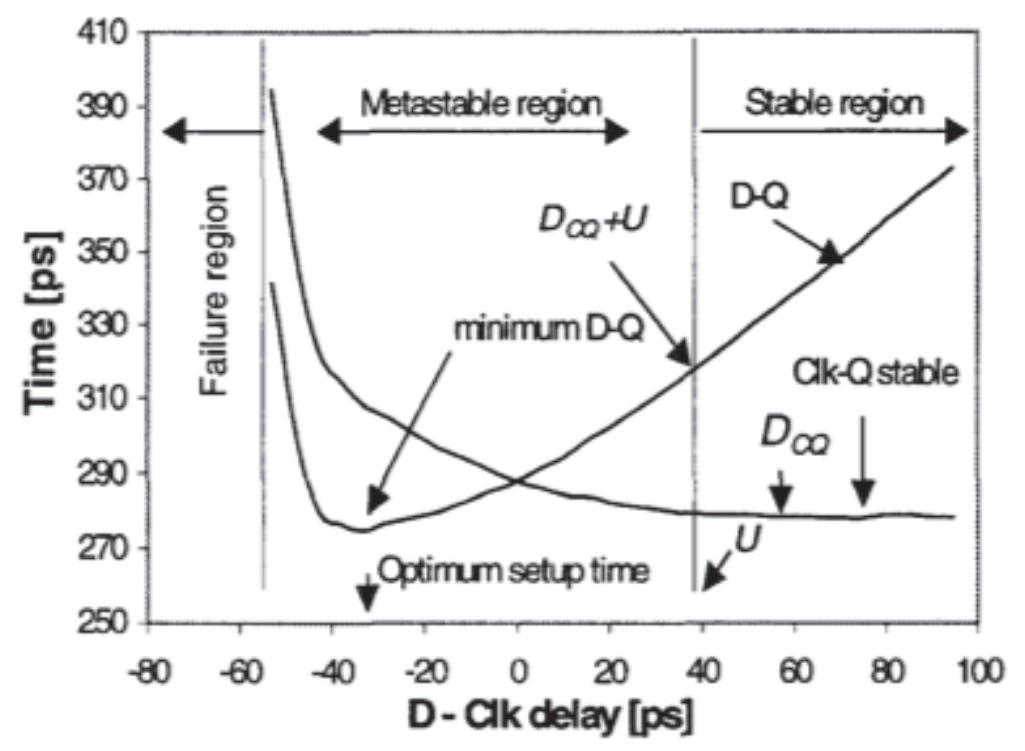

Figure 2.3: Flip flop in metastable state and the x-axis denotes the time taken by the flip flop output to settle after entering into metastable state [2-4] 
A S R latch enters this mode when the two input signals arrive in a small time duration violating the setup and hold times of the latch. The output state is undecided and takes certain time to settle down to a either logic high or low [5]. This poses an issue when it comes to the application of a SR latch as an arbiter as the output can be undecided. Adding a metastability filter eliminates this issue.

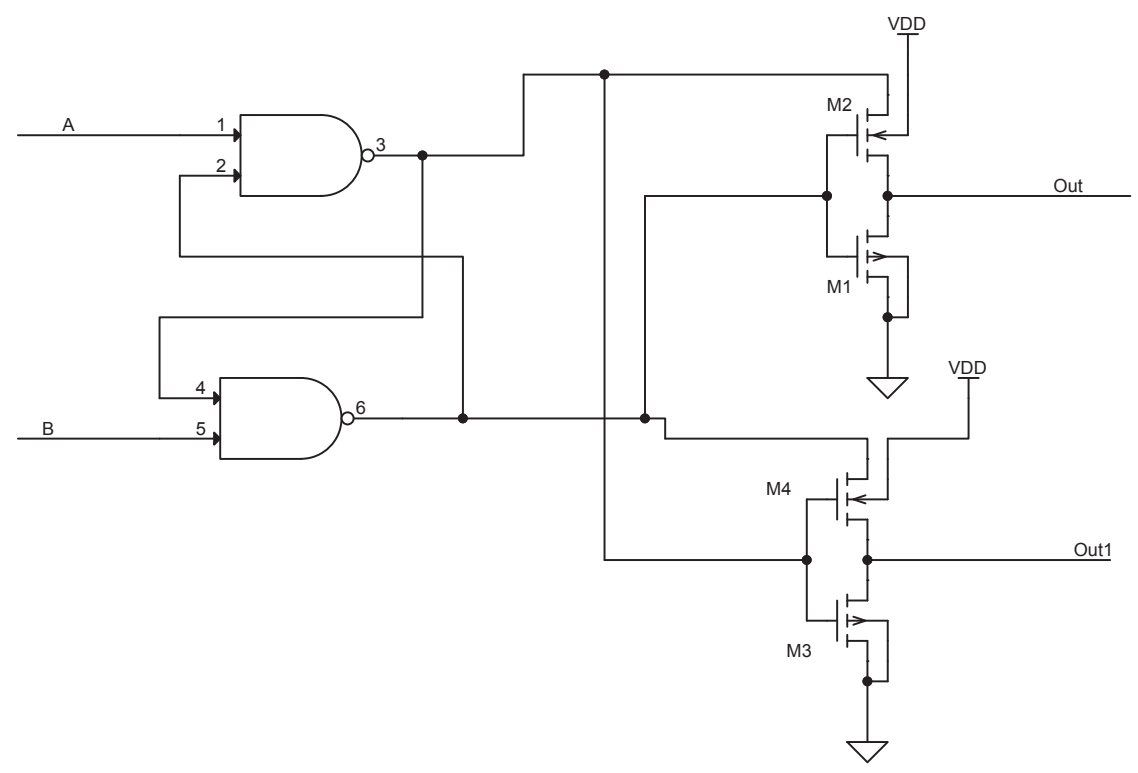

Figure 2.4: Time difference arbiter connected to the metastablity filter realized by MOSFETS, M1 through M4 as shown [6]. 


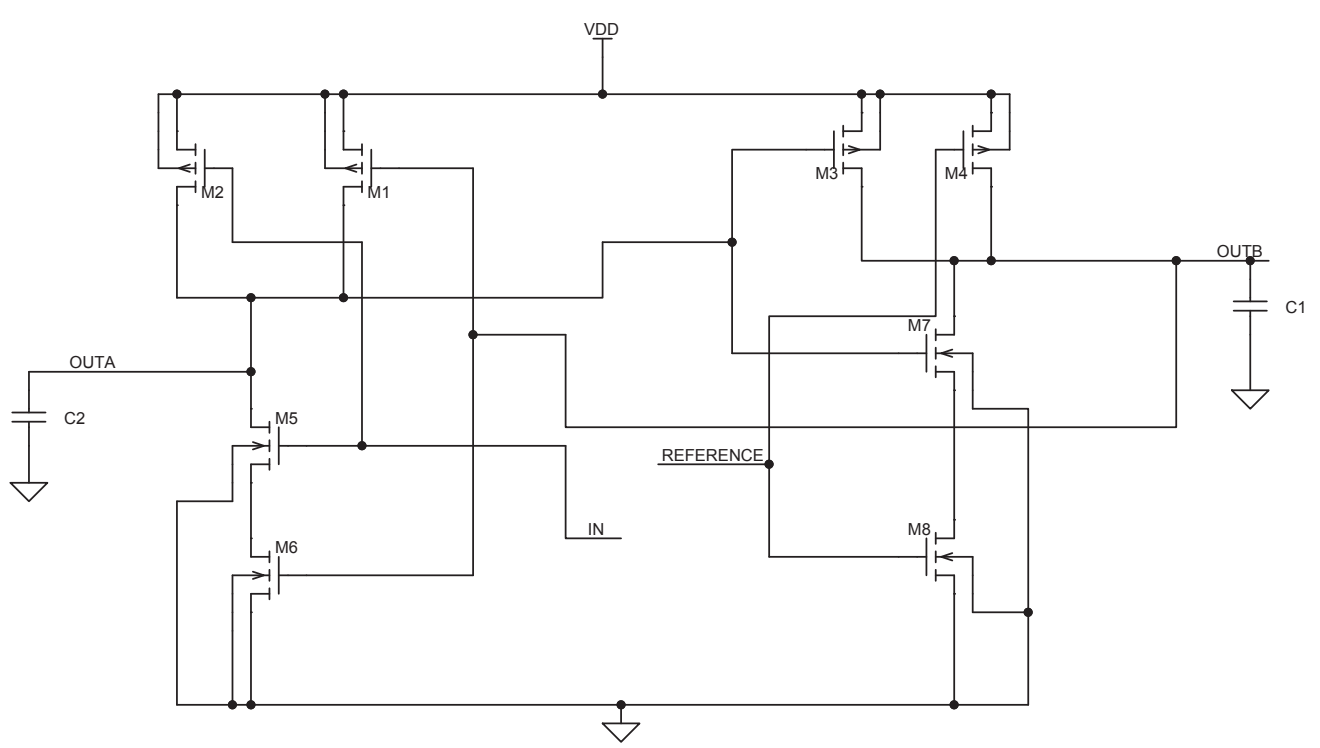

Figure 2.5: Shows a modified arbiter circuit with metastability filter. The filter is realised using capacitors. Transistors M1, M2, M5,M6 form the first NAND gate and M3,M4, M7 and M8 form the second gate.

Image Courtesy [7].

Arbiter circuits are popular and are used for constructing simple time difference amplifiers, they are the main component in case of flash based time to digital convertors, and are used for on chip jitter measurements. [7-9]. 


\subsubsection{Time Difference Adder}

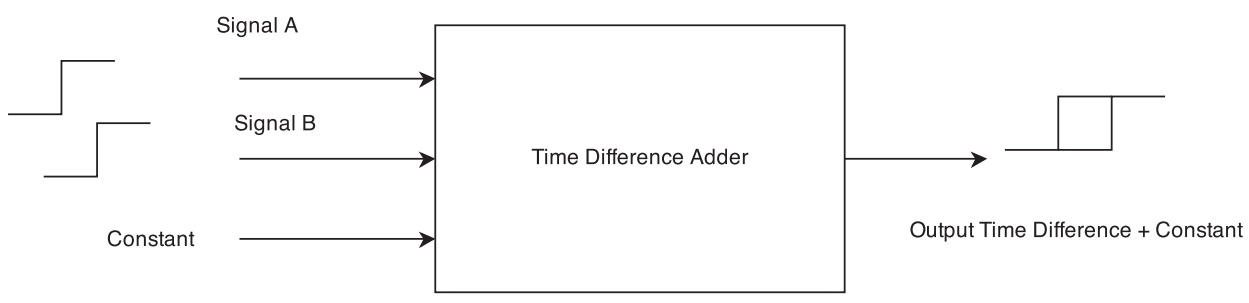

Figure 2.6: Shows a generic block diagram of a Time difference adder where a constant value to the time difference between two signals.

The relation between the input and the output signals are as per the equation,

$$
\Delta T_{\text {out }}=C+\Delta T_{\text {in }}
$$

Where $T_{\text {in }}$ in the time difference between the input signals, $T_{\text {out }}$ is the output time difference and $\mathrm{C}$ is the value of the constant that is added.

A simple implementation of a time difference adder can be an inverter circuit with a fixed capacitor at its output. The capacitor can be added to the output or disconnected from it based on a control signal. When enabled, (control or the adder bit is set to ' 1 ') the capacitor adds a fixed delay of $\tau=R \cdot C$ to the input signal. It should be noted that the propagation delay of the inverter circuit can also add an offset to the input time difference. 

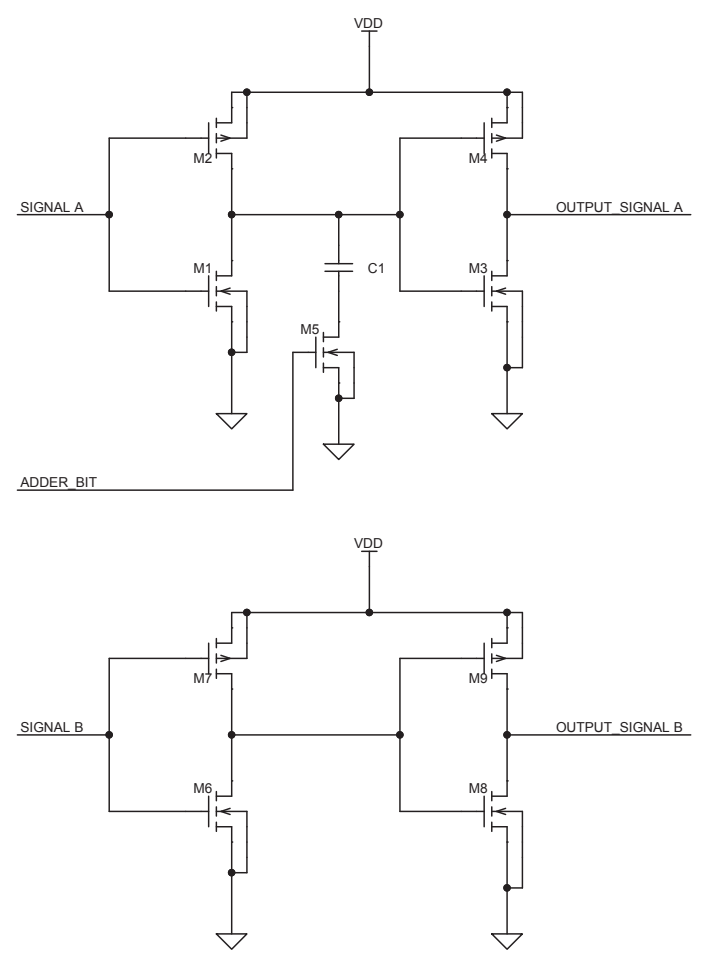

Figure 2.7: Shows an adder implementation of two chains, where the input signals and the adder bit(constant) are applied.

The delay circuit can also be made tunable. Several stages can be added as shown above, where identical capacitors can be added in parallel to increase the delay based on control signals. Capacitors in the arms enabled by the control signal come in parallel and the effective capacitance increases [10] as seen from,

$$
C_{\mathrm{Eff}}=C_{1}+C_{2}+\ldots+C_{\mathrm{n}}
$$

The delay tuner can also be current starved to add additional delay as shown in Figure 2.8. The delay tuner is connected at the nodes of the first inverter chain and the control bit adds or removes capacitors are each node changing the effective capacitance at each node, hence adding a constant to the time difference delay. 


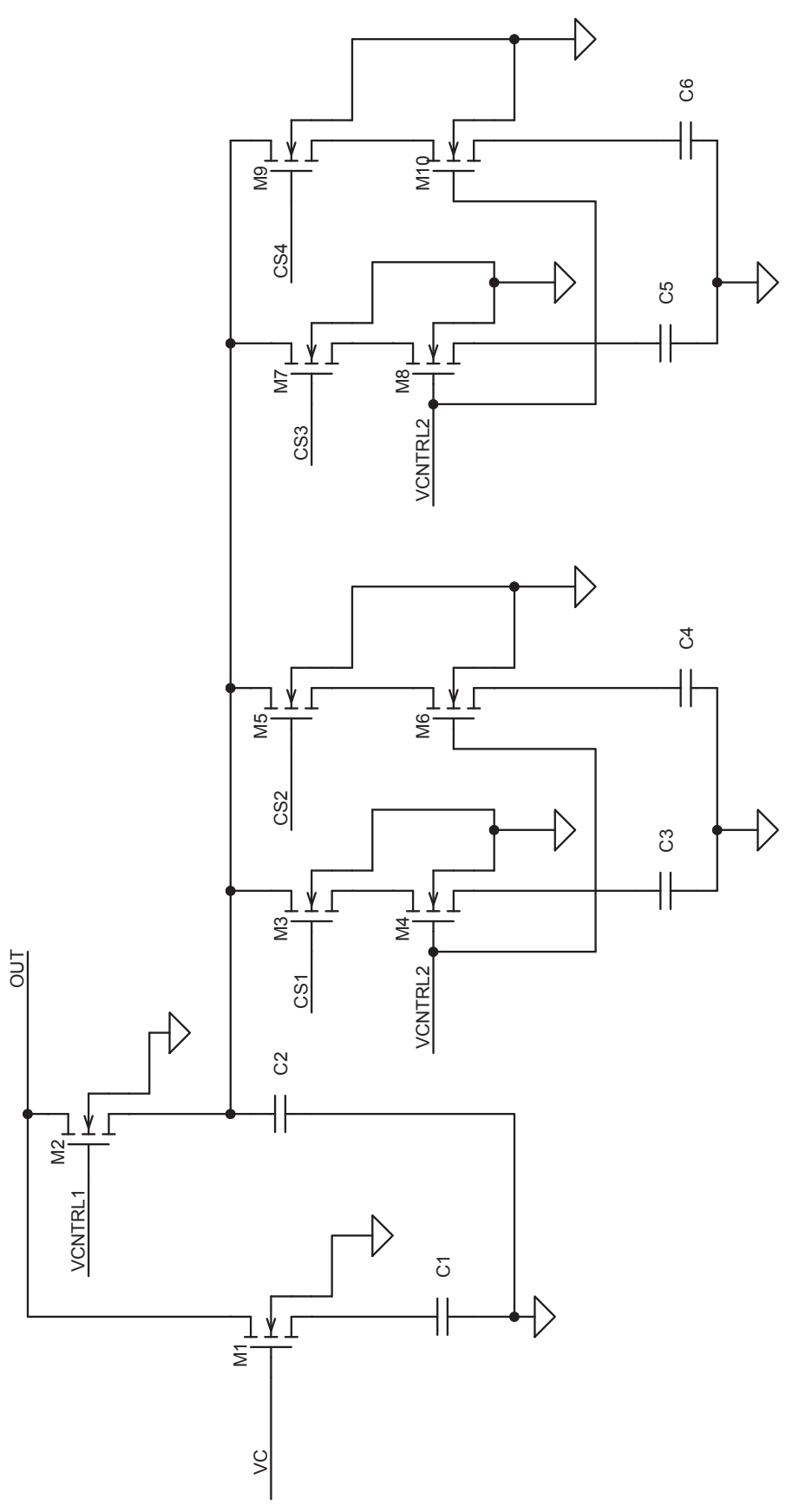

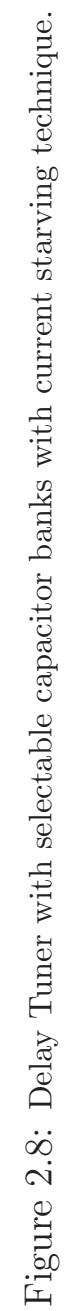


The delay cell is built using capacitors and current starving techniques. The mosfet M1 is current starved to change the charge or discharge rate of the capacitor, hence can change the delay. The mosfets M3, M5, M7 and M9 are used to select the capacitor banks that are added in parallel to $\mathrm{C} 2$ increasing the effective capacitance and hence increasing the delay.

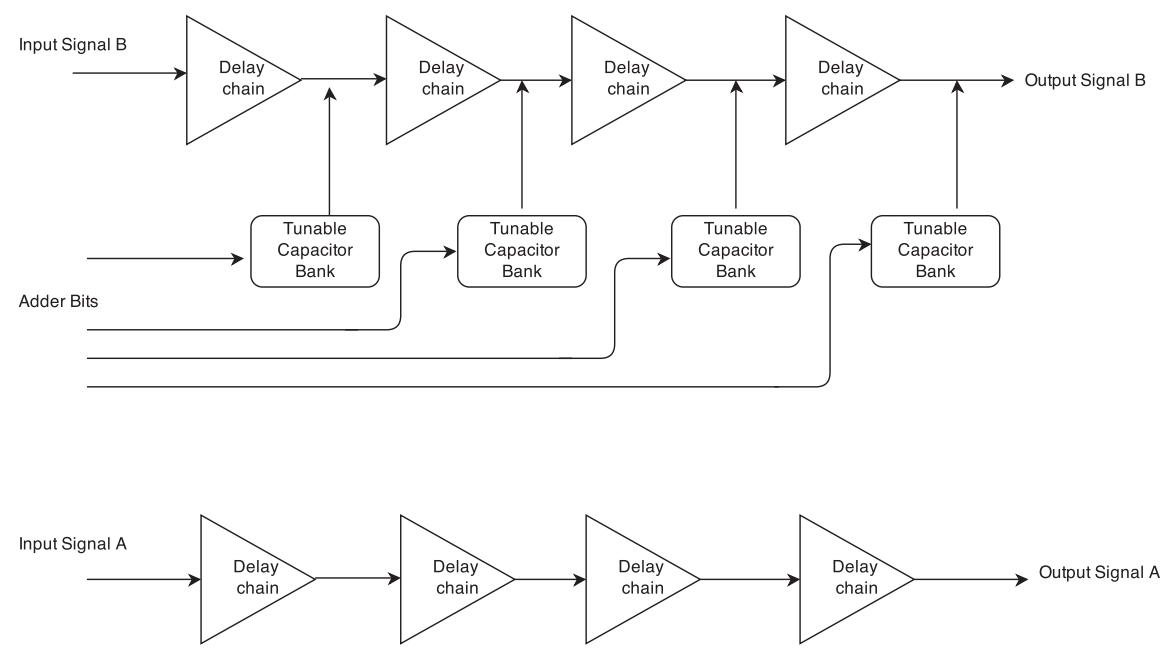

Figure 2.9: Time difference adder block diagram. The delay cells are made of inverters and the tunable capacitor delay cells are added to the output of the cell where based on the adder bit the capacitor array is either added or removed.

Figure 2.9 shows part of the adder chain. The first chain has the capacitor blocks, thus making the delay tunable whereas the second chain is fixed. This constitutes the adder. 


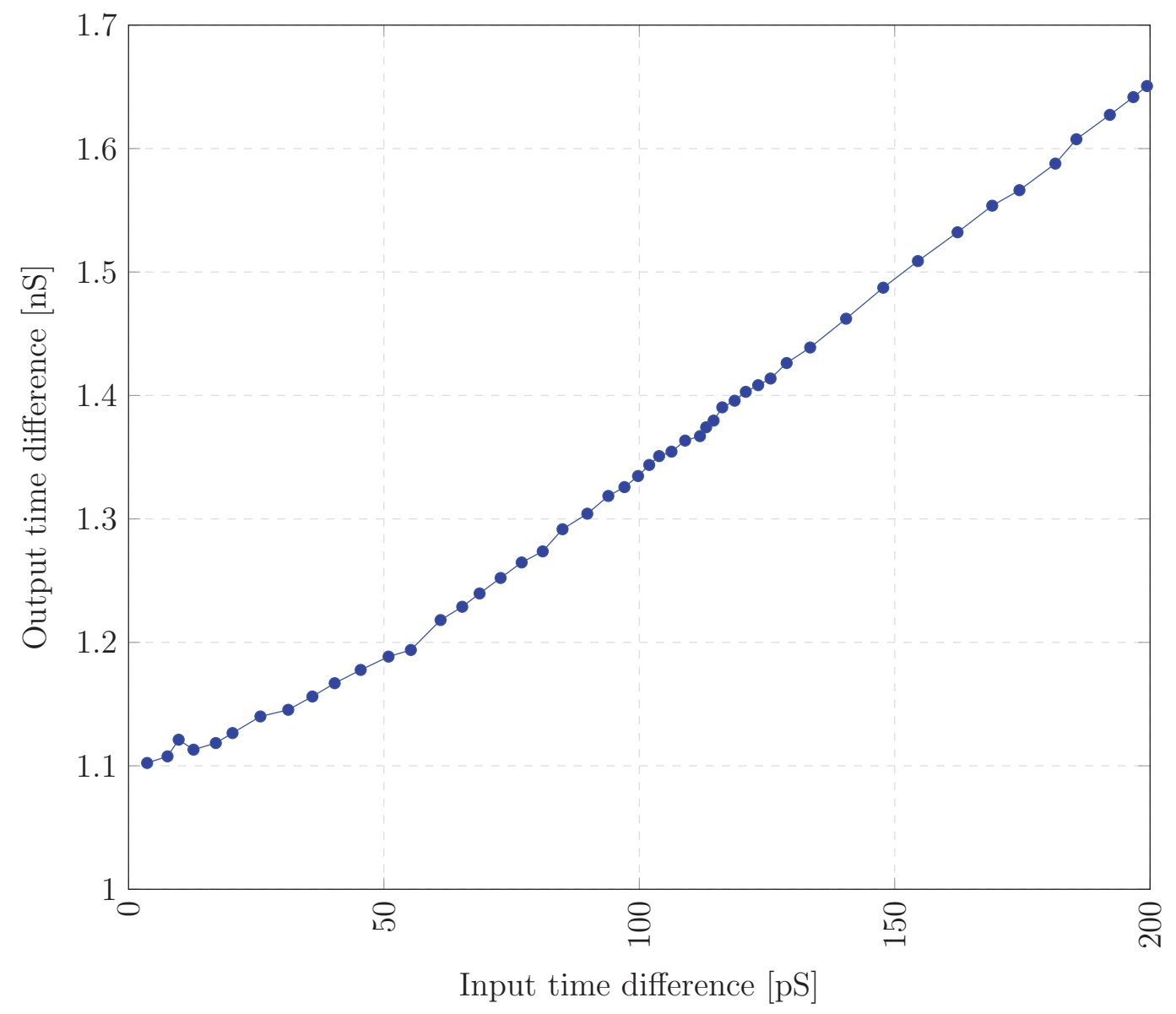

Figure 2.10: Sample plot show the output of the time difference adder, here a constant of 1ns is added to the input time difference.

Figure 2.10 shows the adder plot where the constant is added to the time difference inputs. The input delay is swept and one can observe the constant being added to the input time delay. The time difference adders are typically used in delay lines to provide precise delays [11]. 


\subsubsection{Time to digital Convertors}

A/D converters convert input voltage levels to digital output codes. These codes give the digital value of the analog voltage levels, and these numbers can be easily used by a processor for computations. In the time circuits domain, Time To Digital converters perform a similar operation of providing a digital code equivalent to a time difference and have numerous applications similar to the A/D converters $[1,7-9,12-19,19,20]$.

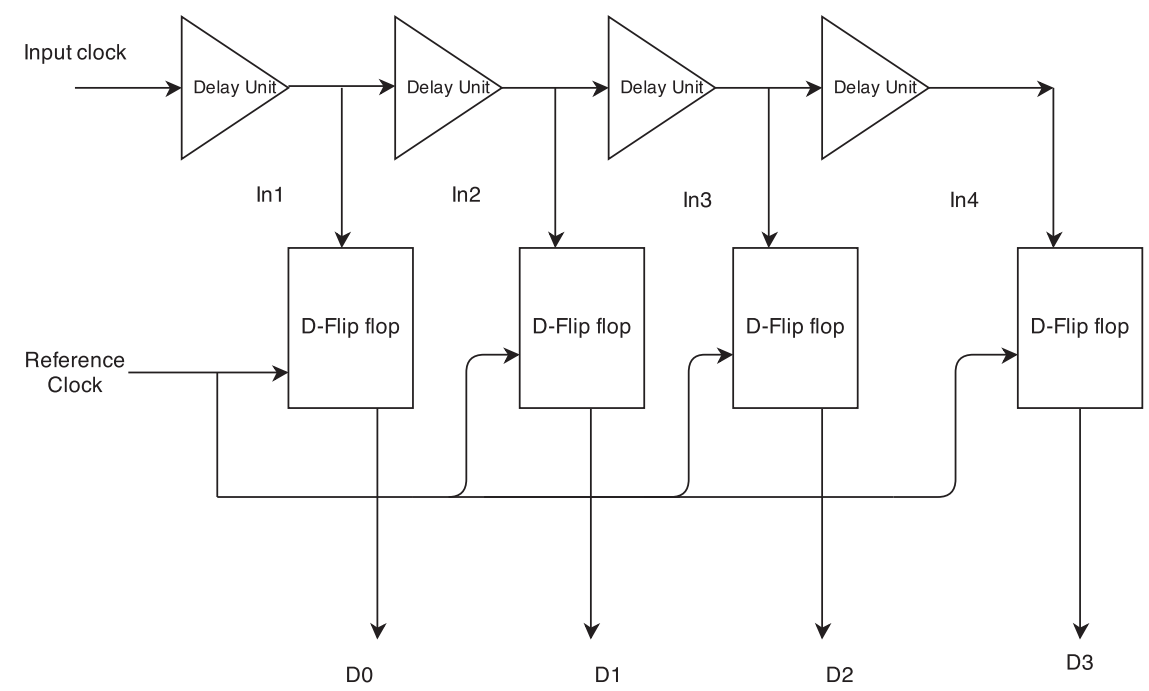

Figure 2.11: Basic TDC architecture is shown, consisting of delay cells where the input clock is fed. The outputs of each delay cell is fed to a flip flop, which compares the signal with a reference clock. This architecture is also called vernier chain based TDC [14].

The basic architecture of time to digital convertor is shown in figure 2.11. The reference clock is a high frequency clock fed to a chain of buffers having minimum delay. The output of each stage is sampled by a Flip flop, clocked at a slower rate. 


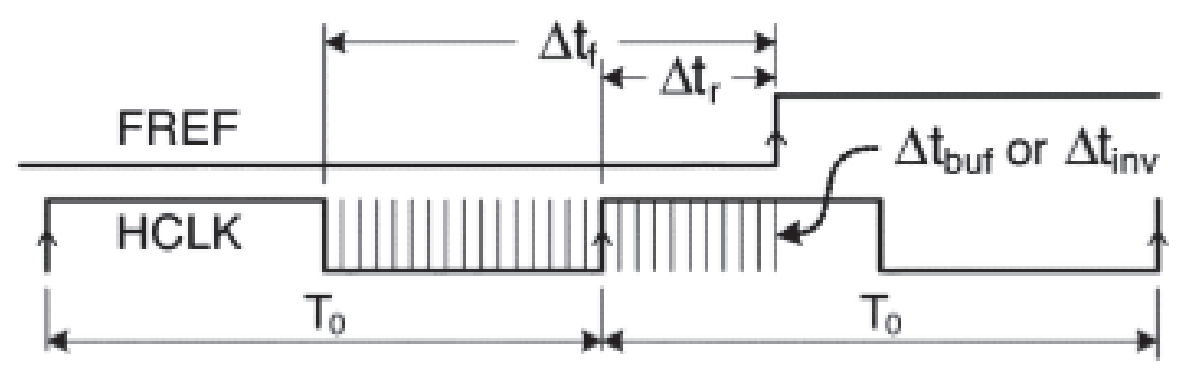

Figure 2.12: Clock input scheme for TDCs [14].

As the HCLK propagates through the chain shown figure 2.12, a fixed delay is introduced at each stage. A rising edge in the reference clock (FREF) latches the data on the output of the respective buffer connected to its input. If the delayed version of the HCLK arrives before the FREF signal at a flip flop then the data it latches will be a logic high and when the FREF arrives before the delayed HCLK then a logic low is latched. The output of all these flip flops, therefore provide a unique digital code representing the time difference between HCLK and FREF. Figure 2.13 shows the detailed waveform from each fixed delay stage.

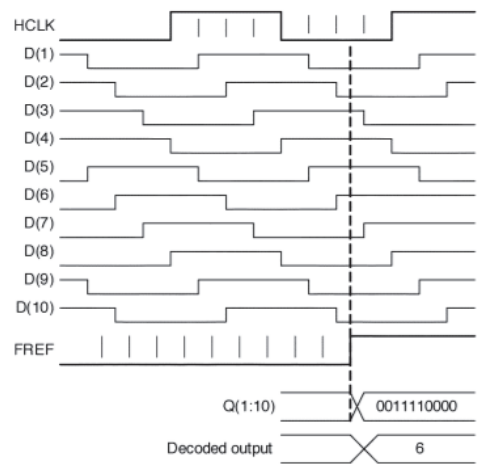

Figure 2.13: Results of the TDC is shown. The digital data equivalent of the input time difference between the input clock and the reference clock is depicted in this waveform. [14].

Integral non linearity (INL) and differential non linearity (DNL) errors determined from the transfer function which tells the deviation of from an ideal transfer 
characterestics which determine the accuracy of the TDC [14].

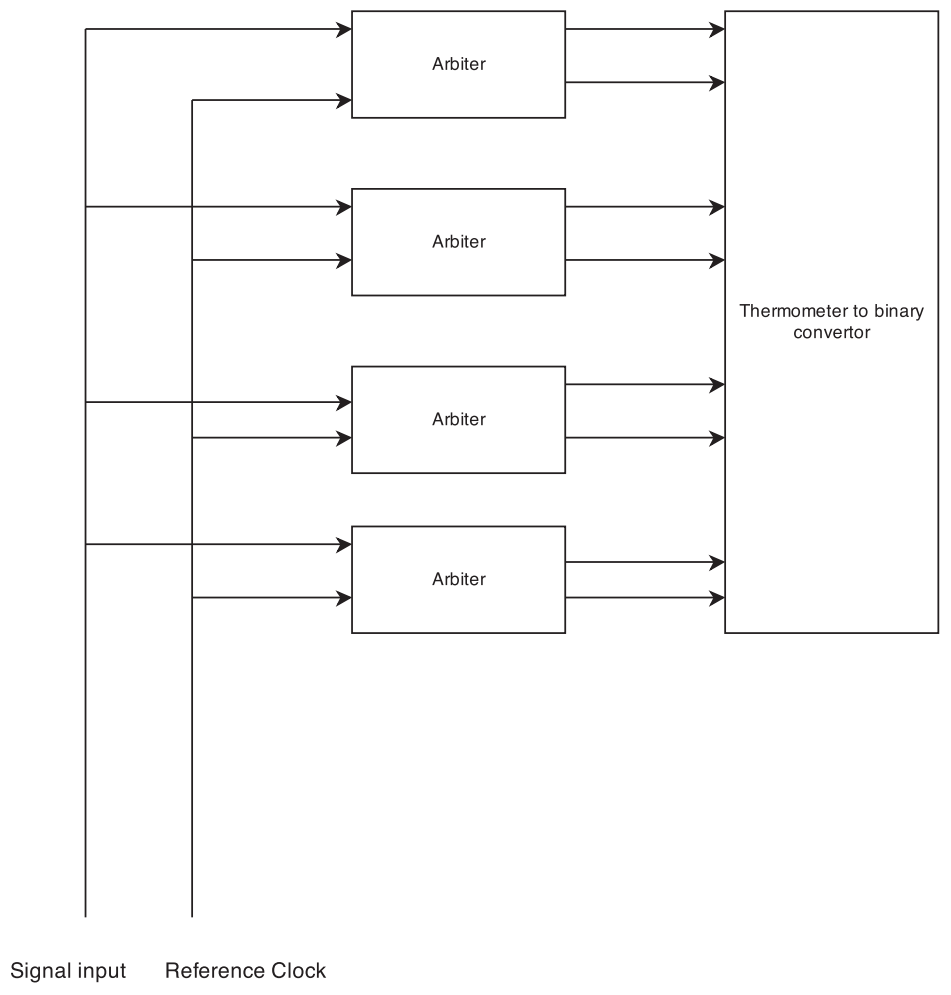

Figure 2.14: Time difference arbiters are used in Flash Time to digital convertors. The architecture is similar to the Flash analog to digital convertors [7].

Arbiter circuits described earlier are used as time difference comparators in flash TDCs shown in figure 2.14. The input signal delayed with the help of precise delay line and a reference signal form the two inputs to an arbiter block. Several such arbiters are used to compare the delayed signal and the reference and provide a suitable output, the delays increase in each stage. The combined outputs of the various stages provide a thermometer code that is converted further to provide a digital code representing the time difference. The resolution of the TDC depends on the number of stages of arbiter. In the figure the size of the NAND gate symbol in each arbiter connected to the actual is representative of the delay applied to it. 


\subsubsection{Digital to time converter}

Digital to time converters are analogous to Digital to Analog converter. These circuits generate output time differences corresponding to the input digital code.

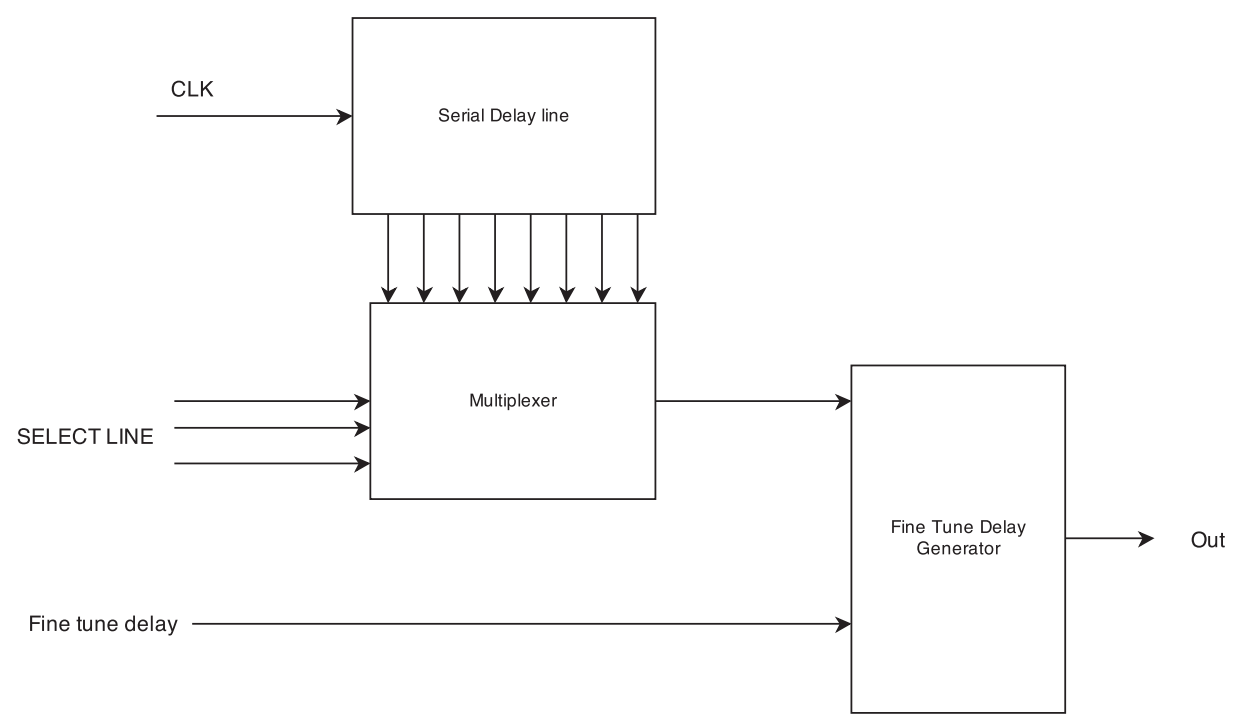

Figure 2.15: The Basic DTC architecture consists of a delay line where the input signal is delayed. The mux is used to select the delayed output based on the code, to generate the output time difference [22].

Figure 2.15 shows a basic DTC. The DTC internally has several cascaded delay lines that provide precise fixed delays. The point connecting two such delay elements are brought out as taps. An N:1 multiplexer is employed to select the appropriate tap based on the input code. While most significant bits of the code can be used for coarse selection using the mux, the least significant bits can also be employed to select fine grained delays using a fine delay generator.

The delay element itself can be realized using the techniques such as varactors or current starving methods. 


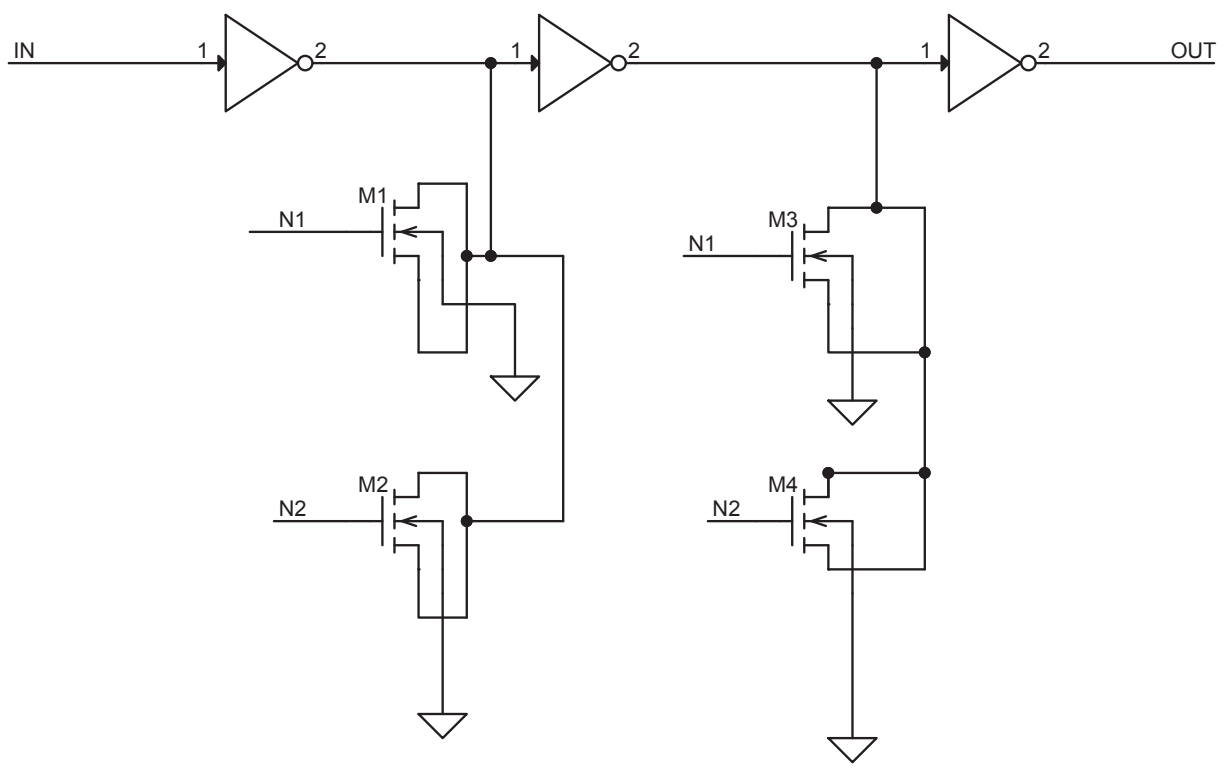

Figure 2.16: A basic building block of a DTC is shown. M1 to M4 are MOSCAPS that are connected to the output node of the inverter chain. The moscaps add capacitance to the output node increasing the slew rate of the signal hence providing the delay [22].

Figure 2.16 shows the structure of the delay cell used in the DTC. In this implementation the transistors behave like capacitors and are connected to different stages in the delay line.

A more complicated DTC is shown in 2.17. Here the DLL helps the circuit to give accurate delays by changing the control voltage, on the fine delay tuner. The control voltage is generated using a charge pump. This scheme is added to provide stability towards process, voltage and temperature fluctuations. 


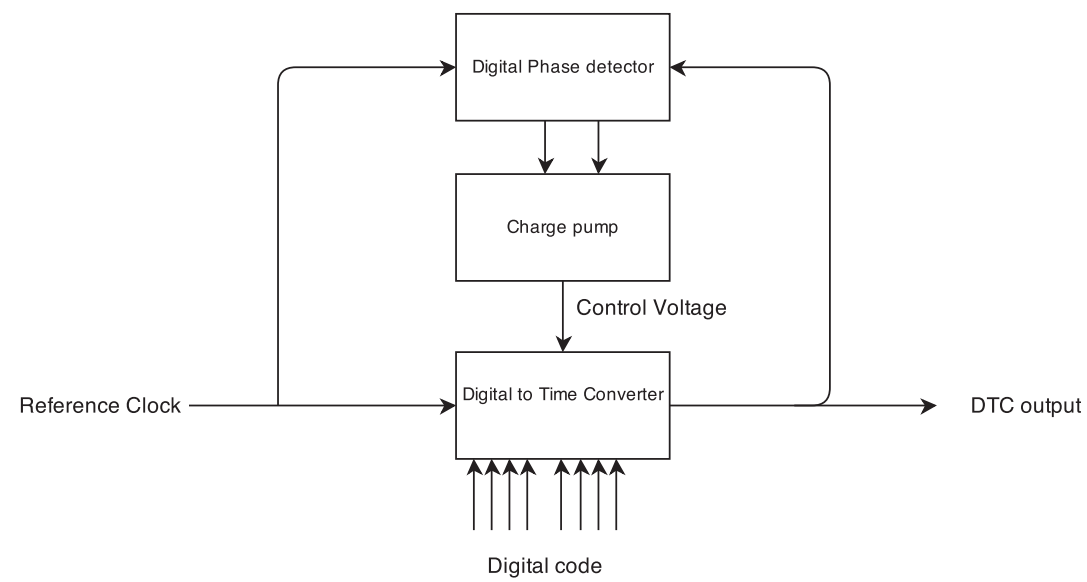

Figure 2.17: DTC is integrated along with a DLL as a feedback mechanism, that corrects the output delay, when subjected to PVT conditions. [23].

The INL and DNL are important specification for the DTC, similar to the TDCs.

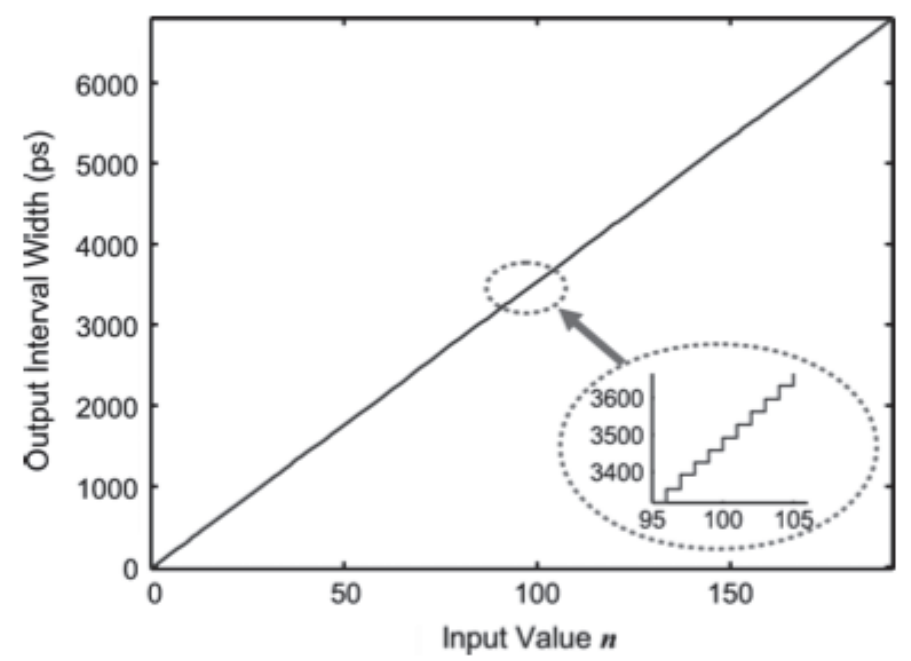

Figure 2.18: The transfer function of a DTC is shown in the above figure [24].

Figure 2.18 shows the transfer characteristics of a DTC, where the output code is represented as a time value [24]. 
The DNL and INL plots are shown below.

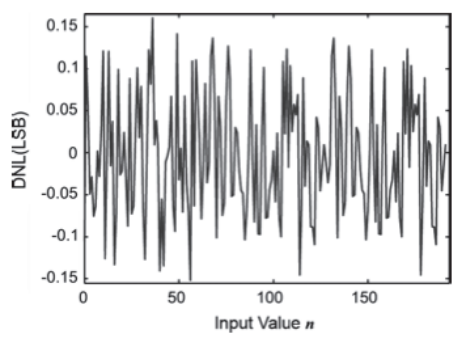

Figure 2.19: DNL plot for DTC is shown [24].

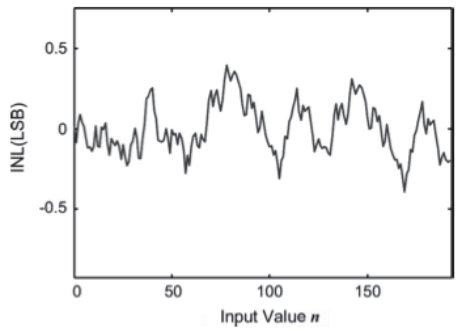

Figure 2.20: INL plot for DTC is shown [24].

DTCs are employed in calibrating timing for high speed circuits [22, 25]. 


\subsection{Applications of Time Difference Circuits}

This section briefly describes the applications of the Time Difference Circuits.

\subsubsection{LIDAR}

LIDAR stands for laser imaging detection and ranging. These devices are used for measuring distances, using lasers. They are widely used in construction, agriculture and also in military applications. Time difference circuits are widely used in this application $[26,27]$.

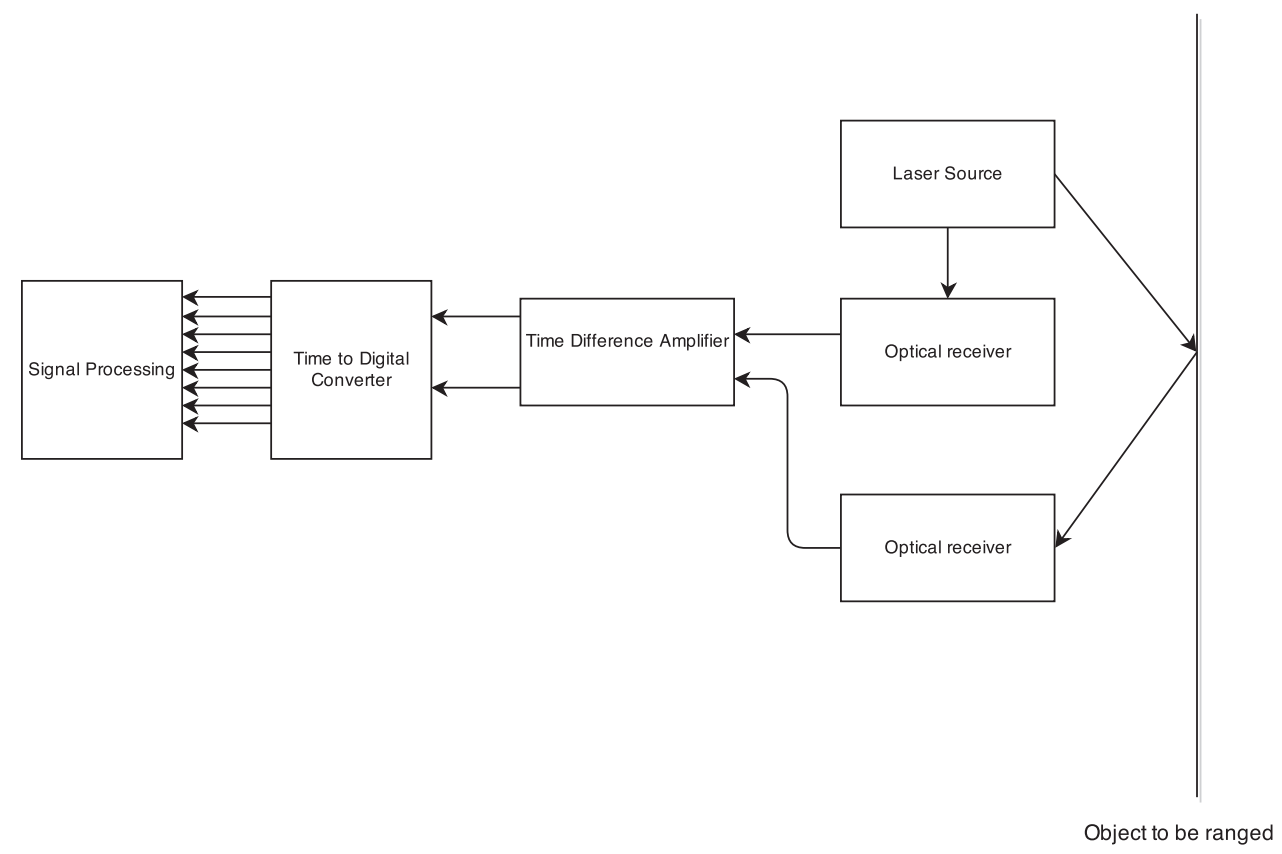

Figure 2.21: A LIDAR system is depicted in this figure. The Laser light is pulsed and this is taken as a refernce signal. Reflected light is captured and processed. The TDA amplifies the time difference between the reference and the received signal. The TDC converts the time difference to digital codes later can be processed. Arrows indicate the signal flow.

Figure 2.21 shows a basic block diagram of a LIDAR system. A laser light is pulsed to find the range of the object. The light is reflected from the light and is incident on the detector. The detectors convert the light energy to the 
electrical energy, the time difference between the emitted pulse and the received pulse is measured using a a time difference circuit, amplified and then converted to a digital code using a TDC. Since the speed of light is known the distance or range can be calculated using the elapsed time.

\subsubsection{Fluorescence}

Fluorescence imaging is used in the medical field for diagnosing cancer, AIDS and disorders that are undetectable using normal diagnosis. In this method the sample is first injected using a dye called fluorophore. The sample is then exposed to light of a particular wavelength, which are generated by lasers. The fluorophore absorbs the light energy first and then re-emits the same [28-30].

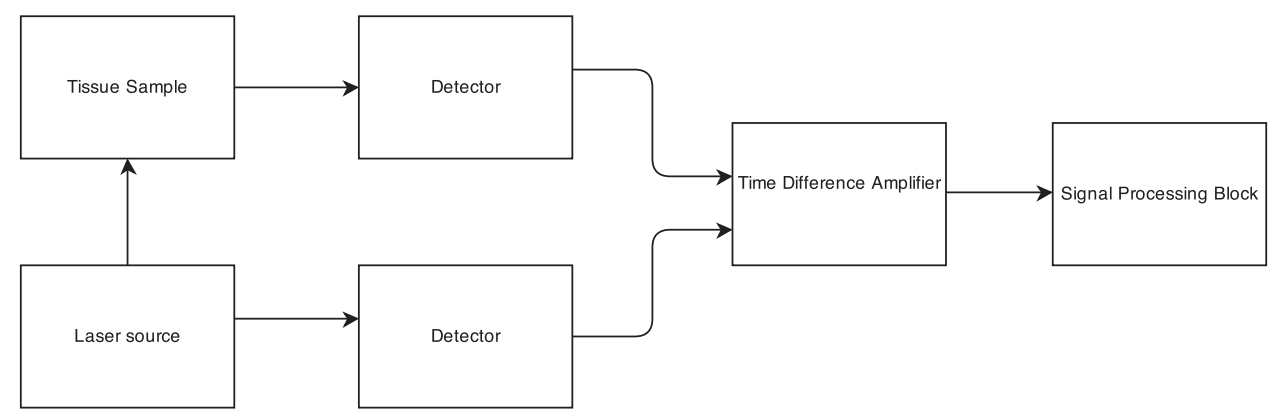

Figure 2.22: The figure depicts the fluorescence system employing Time Difference amplifiers. The tissue is subjected to a laser pulse, the refence signal and the input is fed to TDA to amplify the time difference and is then fed to processor for post processing.

Capturing of the reemitted light can be done in many ways. A CMOS Charged Coupled device or a photo detector can be used to capture the event. For imaging purposes CCD elements are used [29]. Time difference circuits are used as building blocks for fluorescence experiments. This thesis focuses on the design of the time difference amplifier specific to this application. 


\subsubsection{Timing analysis for high speed circuits}

Time difference circuits are also used for on chip jitter measurements and also correction of setup and hold time violations in high speed digital circuits. [8,31-33].

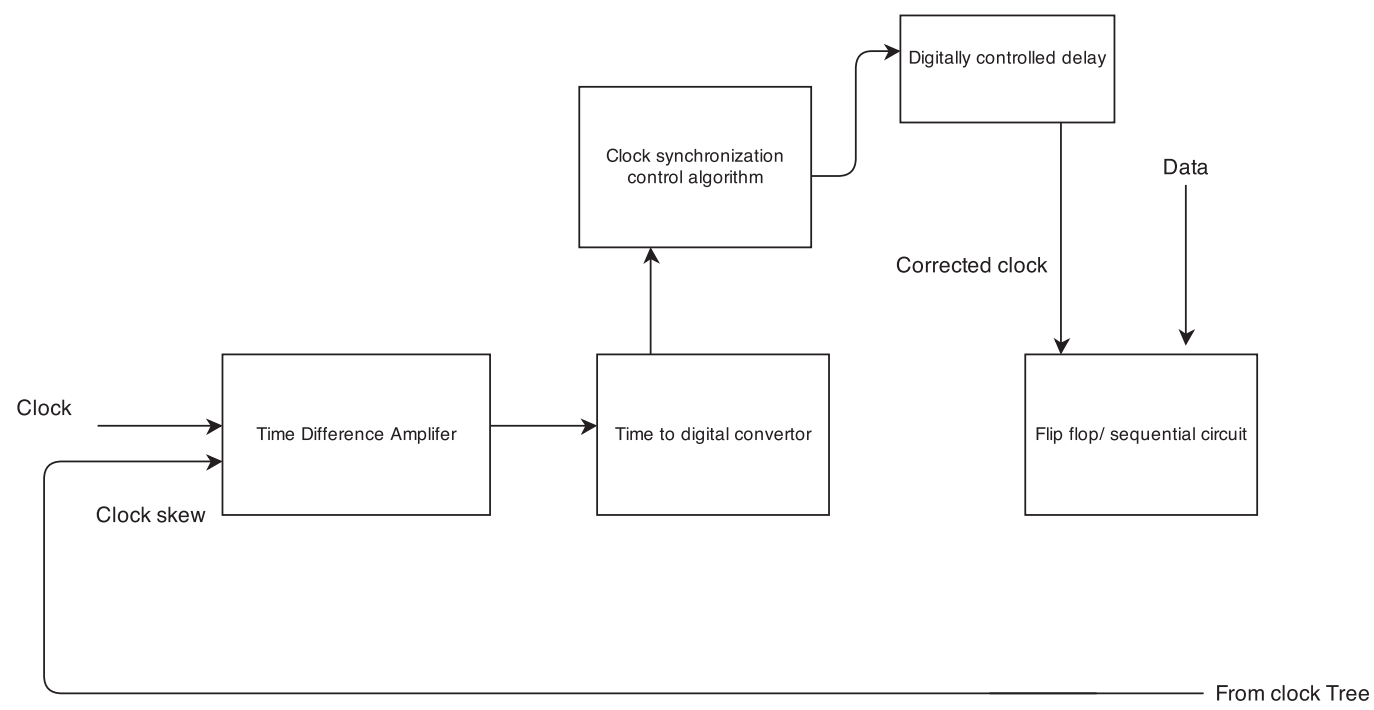

Figure 2.23: On chip jitter measurement block diagram using a time difference amplifier and a time to digital converter [33].

Figure 2.23 shows the applications of time difference circuits used for on chip time measurement on high speed circuits. Vernier based time difference circuits are popular here.

\subsubsection{Direct Digital Synthesis}

Direct Digital Synthesis is a process of generating waveforms such as sine, square, ramp etc.. , they are used widely in radio systems and impedance measurement circuits $[34,35]$. This block can produce sine waveform of a frequency. The frequency of the waveform can be programmed by changing the value in the digital register. The frequency change is achieved by using an Numerical controlled oscillator (NCO) [34]. 
In a phase interpolation based DDS utilizes a Digital to time convertor and a phase accumulator to produce square wave whose frequency is programmable. Upon filtering the square wave a sine wave can be generated [22]. DDS circuits are popular in impedance measurement circuits.

\subsubsection{Particle Research for photon Counting}

The Time difference circuits are used for photon counting applications $[26,30]$.

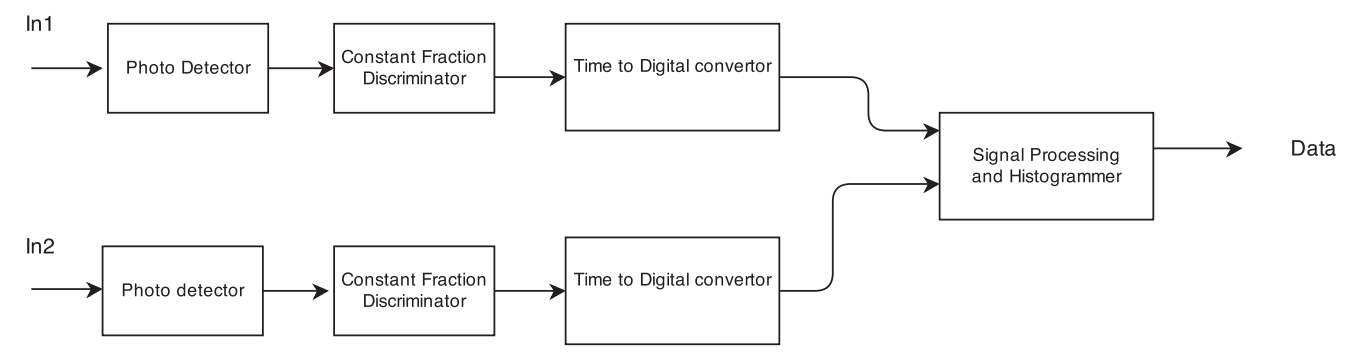

Figure 2.24: The photo detector converts the incident photon to electrical signals. The time information from these signals are extracted by the constant function discriminator. The processed time difference is digitized by a time to digital converter. The data is further processed by the signal processing block [30].

Figure 2.24 shows the basic architecture of photon counting system. Photon counting experiments are used in particle physics. The incident signals In1, In2 are fed to the photo diode and are filtered and pulse shaped. The two inputs are fed to a time to digital converter, which gives a digital value with respect to the time difference between the signal. The data is post processed, to estimate the photon count.

This chapter has given a birds eye view of different time difference circuits and their applications. 


\section{Chapter 3}

\section{Time Difference Amplifiers}

This type of the time difference circuit that is the basis of this thesis is discussed here in detail. The various architectures possible, their merits and demerits are covered here. As the name signifies time difference amplifiers are used to provide a gain to the time difference between two signals while maintaining good linearity. The time difference Between the incident signals are typically in the order or pico seconds, gain and other parameters of the amplifier are application dependent. A block diagram of a typical TDA is shown below.

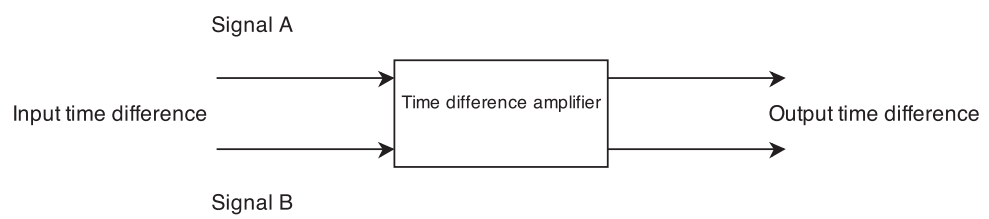

Figure 3.1: Shows a basic block diagram of a time difference amplifier. 
Two input signals $\mathrm{A}$ and $\mathrm{B}$ are fed with the time delay between them as $\Delta t$ and the output is $A \cdot \Delta t$ where $A$ is the gain of the amplifier. The performance metrics for TDA, like any other amplifier, are the gain and the linearity. While the gain metric decides how much the input time difference is amplified by, the linearity tells us how constant the gain is, throughout the amplifier's input range.

While there are different architectures available for implementing these TDAs and all of those are described herein, the main motivation in this thesis is to address the issue of linearity in Vernier chain based time TDAs.

\subsection{Architecture Overview}

TDAs can be implemented in a variety of ways.

- Switched capacitor based time difference amplifier

- Mutex based Time difference amplifiers

- Variable delay chain based Time difference amplifiers 


\subsubsection{Switched capacitor based time difference amplifier}

This architecture is based on Switched capacitors. The schematics are shown in figure 3.2 .

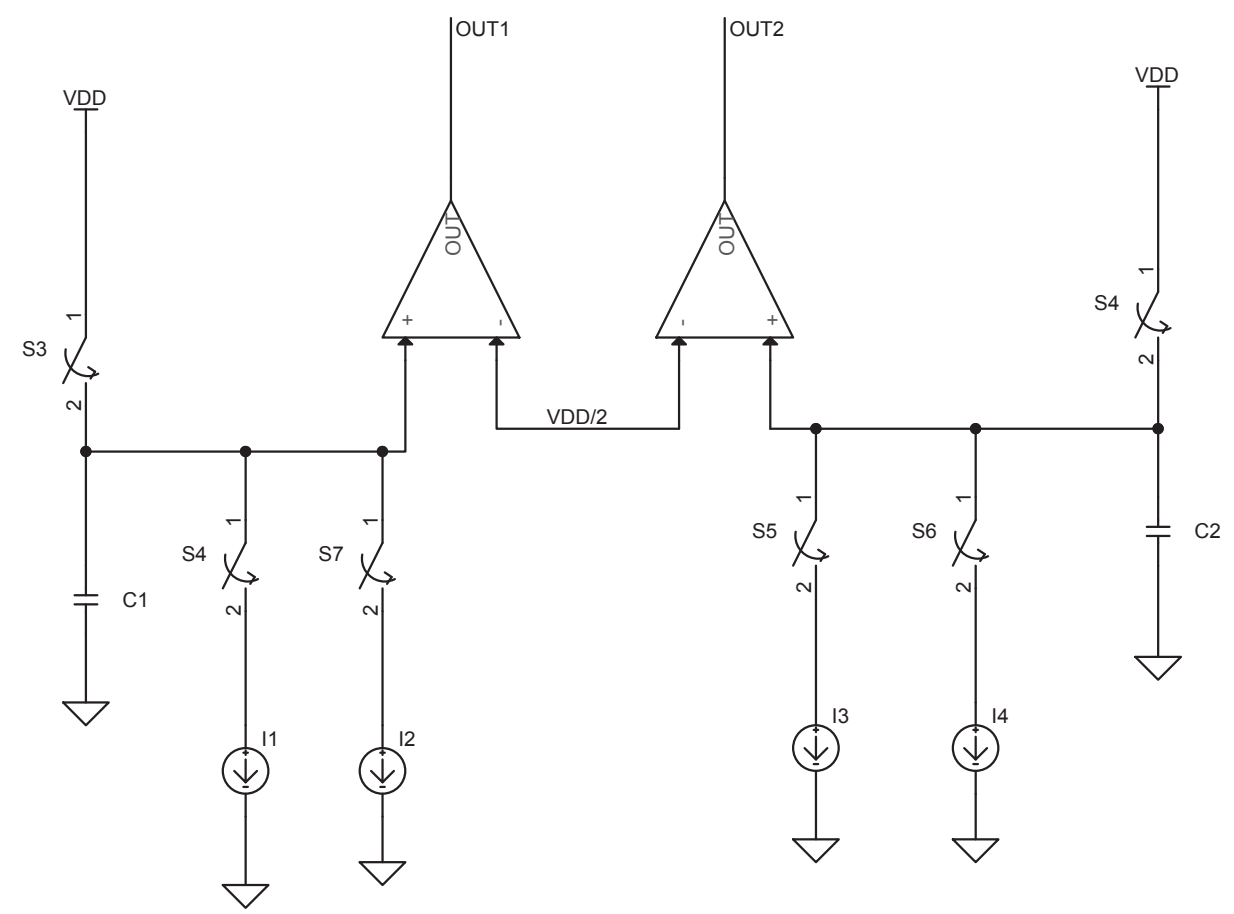

Figure 3.2: The two comparators are connected to a switched capacitor circuits. The comparator compares the voltage at half the supply. The switches are activated by a combinations of the input signals [20].

The capacitors $\mathrm{C} 1$ and $\mathrm{C} 2$ are initially charged to the supply rail and based on the states of the input signals which operates the switch S3 and S4. They are discharged through constant current sources I1, I2, I3 and I4. The voltage on the capacitors which are ramp signals are compared with a reference voltage which is half the supply voltage. The output of the comparators represents the amplified time difference between the two signals. The waveform is shown in figure 3.3. 


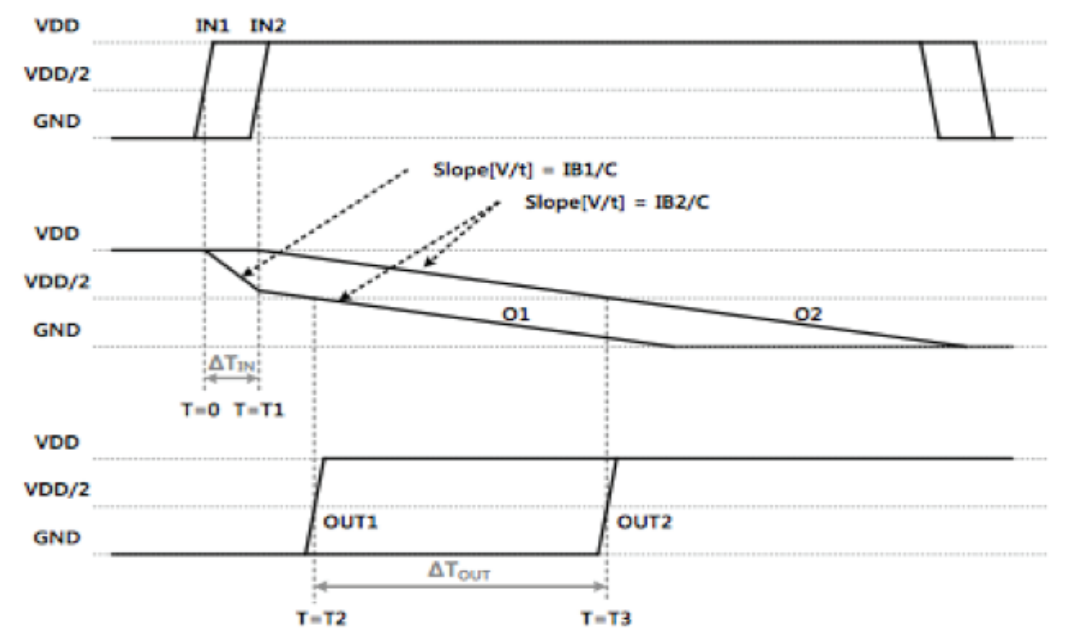

Figure 3.3: The two comparators are connected to a switched capacitor circuits. The capacitors are precharged when the signal input is zero. The capacitor is discharged at a constant rate when the input 1 changes from logic 0 to logic 1 , similarly the second branch . The comparator compares the voltage at half the supply, which gives the time difference [20].

This particular architecture suffers certain drawbacks. The capacitors add an offset to the output signal, the error in gain is larger compared to other architectures.In terms of circuit layout matching capacitors C1 and C2 are important and that determines the linearity of the amplifier. The hysteresis of the comparator is also a factor that affects linearity [20].

However it is possible to achieve higher gain with a single stage with this kind of amplifier compared to the vernier chain architecture and it is easier to tune the gain of the stage by changing the bias current . 


\subsubsection{Mutex Based Time Difference Amplifier}

\section{Metastability in Flip Flops}

Metastability is a state that occurs in a SR flip flops when the setup and hold time requirements are not met. During this state the output of the flip flop is undecided and the output can oscillate a between logic 0 and logic 1 before it settles to a stable state. Table 3.1 shows the various states of a RS flip flop

\begin{tabular}{|c|c|c|c|c|}
\hline $\mathrm{S}$ & $\mathrm{R}$ & $\mathrm{Q}$ & $\mathrm{Qb}$ & State \\
\hline 0 & 0 & Previous state & Previous State & Hold State \\
\hline 0 & 1 & 0 & 1 & Reset State \\
\hline 1 & 0 & 1 & 0 & Set State \\
\hline 1 & 1 & 1 & 1 & Forbidden State \\
\hline
\end{tabular}

Table 3.1: S R Flip Flop Truth Table

The forbidden state is usually avoided in many of the digital designs. However the MUTEX based time difference amplifier uses this Metastability state of the Set Reset flip flop [33], [36], [37]. The SR flip flop has cross-coupled NAND gates whose inputs are connected to the input signal and a reference signal. When these inputs are applied to the SR flip flop with the time difference between the two being less than the required setup and hold hold times of the flip flop, it enters the metastable state until finally the cross coupled transistor logic latches the state. The circuit takes some time to come out of the metastable state which creates a delay. This delay being the required amplification of the time difference between the input signals depicted in figure 3.4 . 


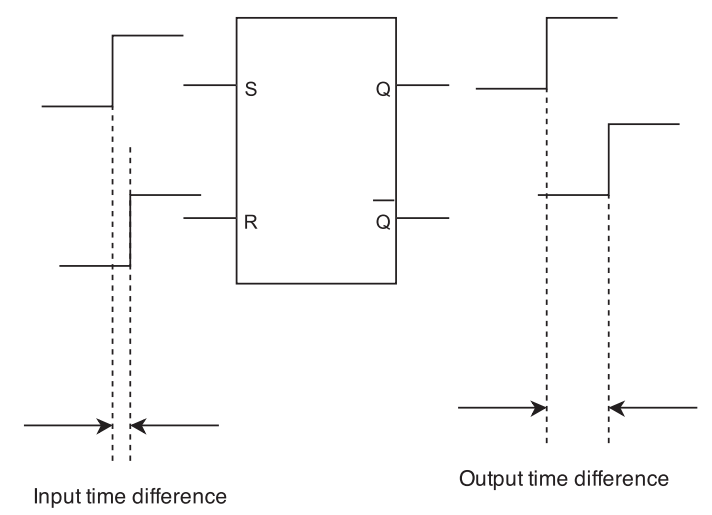

Figure 3.4: An SR latch acts as an arbiter, when the setup and hold times are violated, when operated in the forbidden state, and the flip flop enters a metastable state, the output is undecided for a small time and then either falls to either logic level, this provides the output time difference [33].

The main drawback with the mutex based time difference amplifier is that its linearity is limited to small input range upto 100pS [33]. The plot shown below can be seen variations of output time difference when the input time difference value is swept between a range.

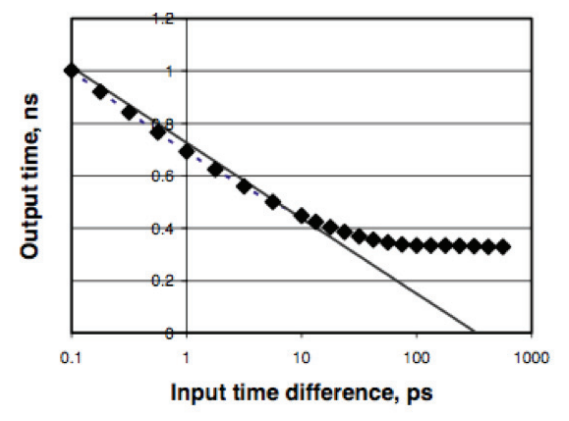

Figure 3.5: Plot showing the transfer characteristics of the mutex based TDA [33].

As the input time is varied from $1 \mathrm{pS}$ to $1 \mathrm{nS}$, the output time difference drops over the range of values, leading to a non-linear gain over an extended input range show in figure 3.5 . 
In other words the MUTEX based TDA can be used over a limited dynamic input range. The main advantage of a MUTEX based TDA compared to an inverter chain based TDA is the area on chip. Inverter chain based TDA's being long and hence take up more area on the chip.

\section{Mutex based TDA with Metastability filter}

In this variant is added a metastability filter at the output of the $\mathrm{S} R$ flip flop.

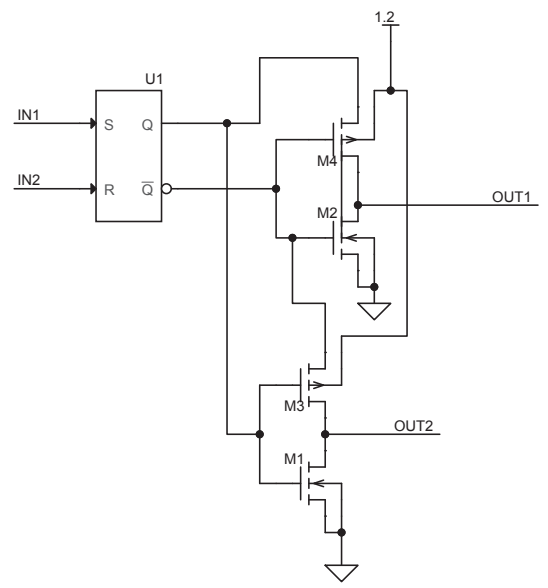

Figure 3.6: The mutex circuit is connected to a metastability filter. Transistor M1 and M3 form one filter, M2 and M4 form the other filter [38] .

Figure 3.6 shows the S-R flip flop which acts as an arbiter. The cross coupled transistors forms the metastability filter. The relationship between the metastability time and the aspect ratio of the transistor is,

$$
t_{\mathrm{m}}^{\infty}=-\left(\frac{L}{W}\right) \cdot C_{\mathrm{tot}} \cdot \ln \left(\theta \cdot \frac{\Delta_{\text {input }}}{\Delta_{\mathrm{v}}}\right)
$$

where, $t m$ is the metastability time measured in second, $\frac{L}{W}$ is the aspect ratio of the transistor, $\theta$ is the time taken for the flip flop to detect initial voltage at the metastable node, $\Delta_{\mathrm{t}}$ is the input time difference in second, $\Delta_{\mathrm{v}}$ is the voltage level 
difference at the output node. $C_{\text {tot }}$ is the total capacitance at the nodes [38], [36], [39].

The output time difference is,

$$
\Delta T_{\text {out }}=\tau \cdot\left[\ln \left(T_{\text {offset }}+\Delta T_{\text {in }}\right)-\ln \left(T_{\text {offset }}-\Delta T_{\text {in }}\right)\right]
$$

where, $\tau$ is the metastability constant. The gain $G$, [38], [36], [15] is given by,

$$
G=\frac{T_{\text {out }}}{T_{\text {in }}}=\frac{2 \tau}{T_{\text {offset }}} .
$$

The metastability constant depends on the $g_{\mathrm{m}}$ of the metastable filter and the $C_{\text {tot }},[36,38]$ given by $\tau=\frac{g_{\mathrm{m}}}{C_{\text {tot }}}$ Varactors are used to tune the delay. Varactors are realized using MOSCAPS where the capacitance can be changed by varying the voltage, this changes the capacitance of the MOSCAP and hence introduces a delay. One can see that by changing $C_{\text {tot }}$ the metastable time can be changed. This results in the change in delay which in turn changes the output time difference and hence the gain. An example is shown in figure 3.7. 


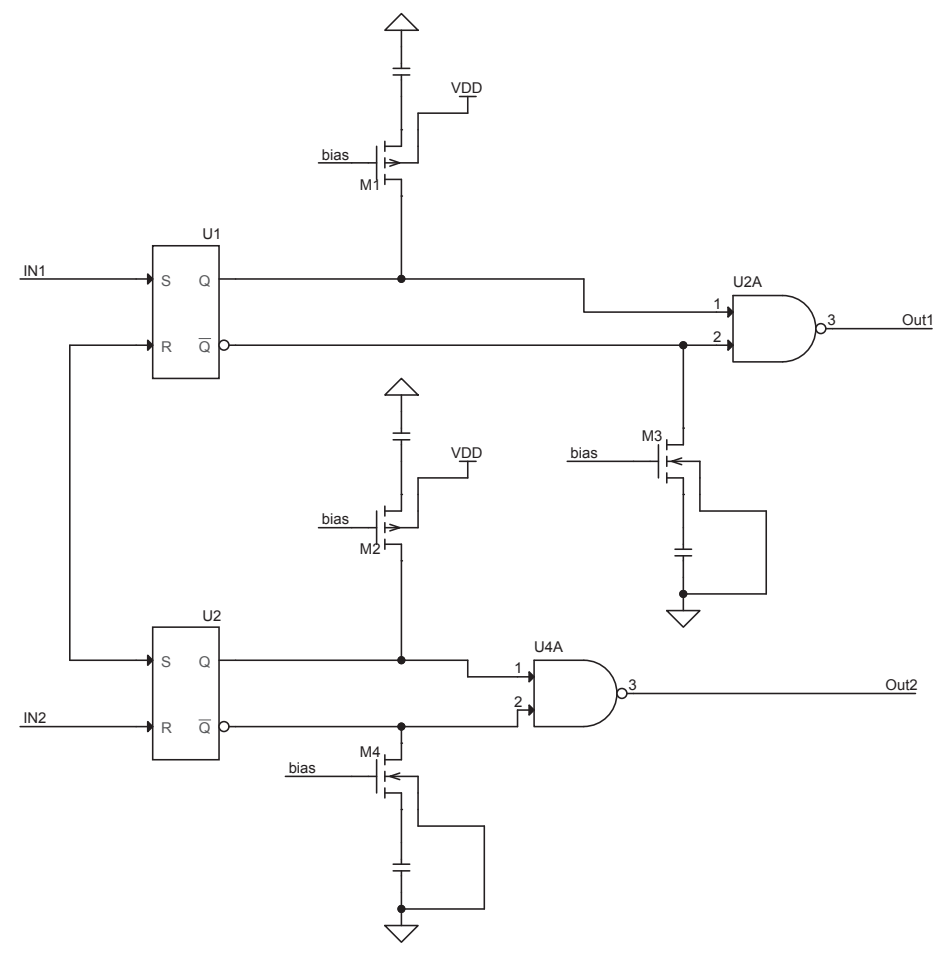

Figure 3.7: Figure shows a variation to the mutually exclusive (MUTEX) circuit with addition of the PMOS and NMOS to control the current through the capacitor. The reason for the limited dynamic input range is due to that fact that when the input time difference between the inputs exceeds the setup and hold times, there will be no violation and the flip flop starts to latch the data $[40]$

\subsubsection{Inverter delay chain architecture}

Figure 3.8 show two delay chains are cross-coupled and when the input propagates through the chain, the output of the delay elements in one chain select the higher delay in the corresponding delay element in the adjacent chain. Ultimately when the signal propagates to the end of the chain all the delay cells will have switched to a higher delay. The vernier delay chain gets the name due to the concept where the two input signals eventually cross each other at a certain time while propagating through the chain. This chain thus acts like a TDA and has a gain associated to it. The gain depends on the number of stages in the chain and also the ratio of the two delays associated with each delay element. The main drawback 


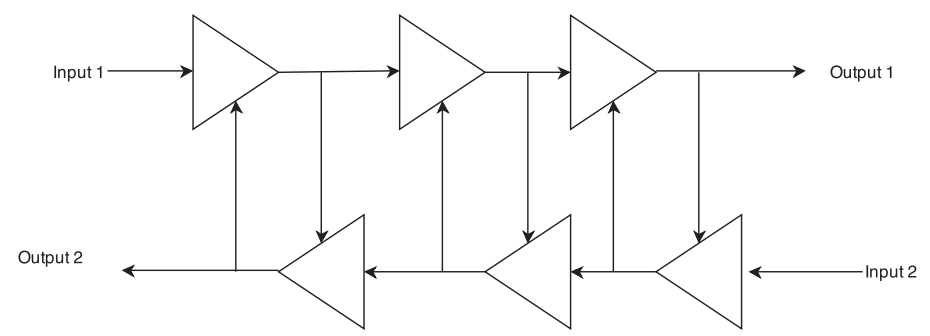

Figure 3.8: Variable delay chain based TDA is a simple architecture where delay elements are cascaded into a chain. Each delay element can provide a long or a short delay based on a select signal.

of this implementation is that when the input time difference is varied the output gets nonlinear and one can see steps in the response indicating variation in the gain. The step response is because of the switching action from a short delay to a long delay. The other drawback of this type of TDA is that the ratio between the shorter delay to a longer delay is fixed. However with minimal changes in circuitry this can be addressed.

The basic block diagram of a delay cell is shown in figure 3.9.

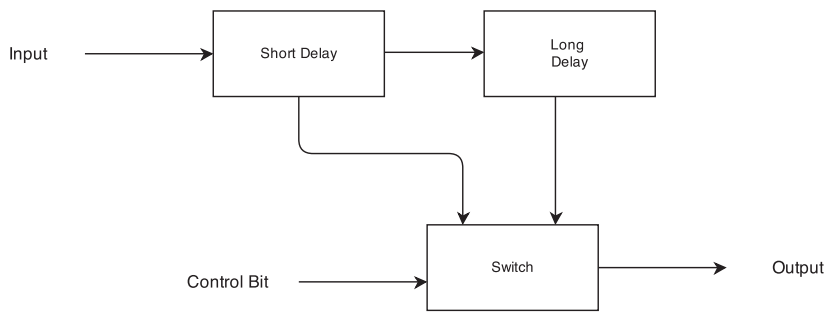

Figure 3.9: The figure is a block diagram of a single delay cell in the chain. The delay cell is split into two components, a shorter delay and a longer delay, the outputs are connected to a multiplexer, the control bit selects whether to select the longer or the shorter delay. 


\subsubsection{Delay Cell Architectures}

Basically Delay cells are inverter circuits that have a propagation delay. The sizing of the transistors in the inverter, determine the propagation delay. The size of the transistor affects the current driven into the load connected to the inverters output. By varying the size and hence the current, the time taken to charge the load at the output can be varied. The load at the output is nothing but the input of the next delay cell.

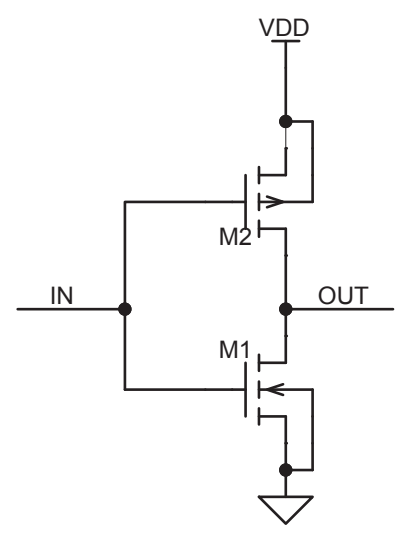

Figure 3.10: M1 and M2 form an inverter, the basic element in the delay cell, the sizing of the M1 and M2 determine the delay. 
To achieve a long and short delay the $\mathrm{W} / \mathrm{L}$ ratio of the transistors are varied. A larger ratio of $W=L$ results in a larger current and hence a shorter delay where as a smaller ratio of $\mathrm{W}=\mathrm{L}$ which results in a smaller current and a larger delay. When the input pulse is applied from 0 to $V_{\mathrm{DD}}$ the $V_{\mathrm{GS}}>=V_{\mathrm{t}}$ for $\mathrm{M} 1$ and $V_{\mathrm{DS}}>$ $V_{\mathrm{GS}}-V_{\mathrm{T}}$, the NMOS is in saturation region and the current is given by,

$$
I_{\mathrm{D}}=\frac{1}{2} \cdot \mu_{n} \cdot C_{\mathrm{ox}} \cdot \frac{W}{L} \cdot\left(V_{\mathrm{GS}}-V_{\mathrm{t}}\right)^{2} \cdot\left(1+\lambda\left(V_{\mathrm{DS}}-V_{\mathrm{DS}, \mathrm{sat}}\right)\right)
$$

Where $\lambda$ is the channel length modulation. The output node discharges through the Nmos transistor and enters into the triode region as $V_{\mathrm{DS}}<V_{\mathrm{GS}}-V_{\mathrm{T}}[41,42]$. From figure 3.10 the problem of having the fixed ratio can be overcome by current starving technique that enables to tune the delay.

\subsubsection{Current Starved Inverter Design}

The drawback in the delay cell design is that the delay is fixed by the $\mathrm{W} / \mathrm{L}$ ratio. The $\mathrm{W} / \mathrm{L}$ ratio being fixed at the time of design and cannot be tuned during operation. This makes the system less flexible.To overcome this drawback we use the concept of current starvation. Often circuit designers in analogue systems talk about "Slew Rate" which defines as rate of change of voltage with respect to time, usually measured in terms of $\frac{V}{\mu S}$. The slew rate is given by,

$$
\frac{d V}{d t}=\frac{I}{C}
$$

where $\frac{d V}{d t}$ is the slew rate measured in $\frac{V}{\mu S}$. I is the output current measured in $m A . C$ is the load capacitance measured in $p F$. The slew rate can be controlled 
by changing the current keeping the load capacitance fixed. If the output current is large then the node is charged or discharged quickly. By starving/controlling the current, the slew rate and hence the delay can be varied. This concept of varying the current to achieve different delays that can be tuned in addition to the inherent propagation delay makes this architecture popular in TDA design.

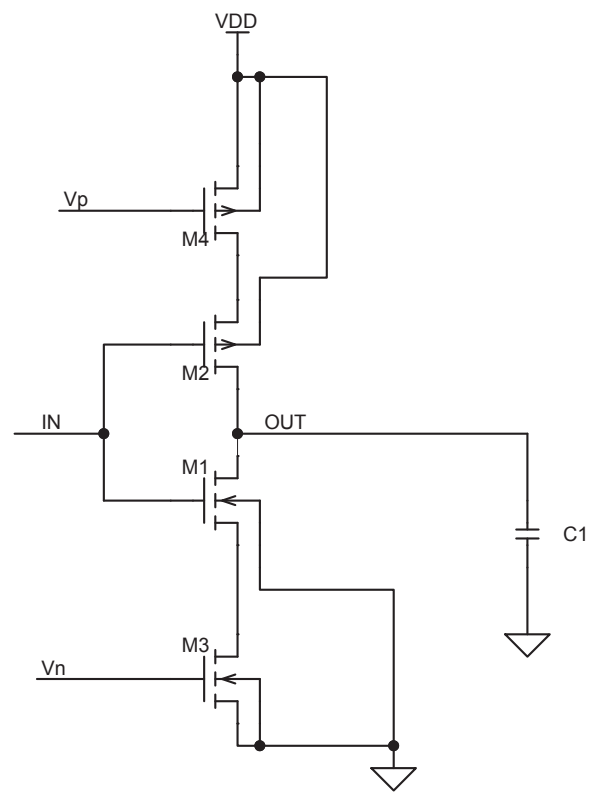

Figure 3.11: Transistors M1 and M2 form the inverter, whereas M3 and M4 are used for limiting the current through the load.

Voltages $V_{\mathrm{p}}$ and $V_{\mathrm{n}}$ are used to tune the current flowing through the load. As the drain current is dependant on the gate voltages applied to M3 and M1, the current through the inverter and hence the Slew Rate can be controlled with $V_{\mathrm{p}}$ and $V_{\mathrm{n}}$. 


\subsubsection{Other Architectures for Delay Cell}

The previous section showed a current starving feature for the inverter. This section describes other techniques and architectures that are used as basic blocks for the TDA.

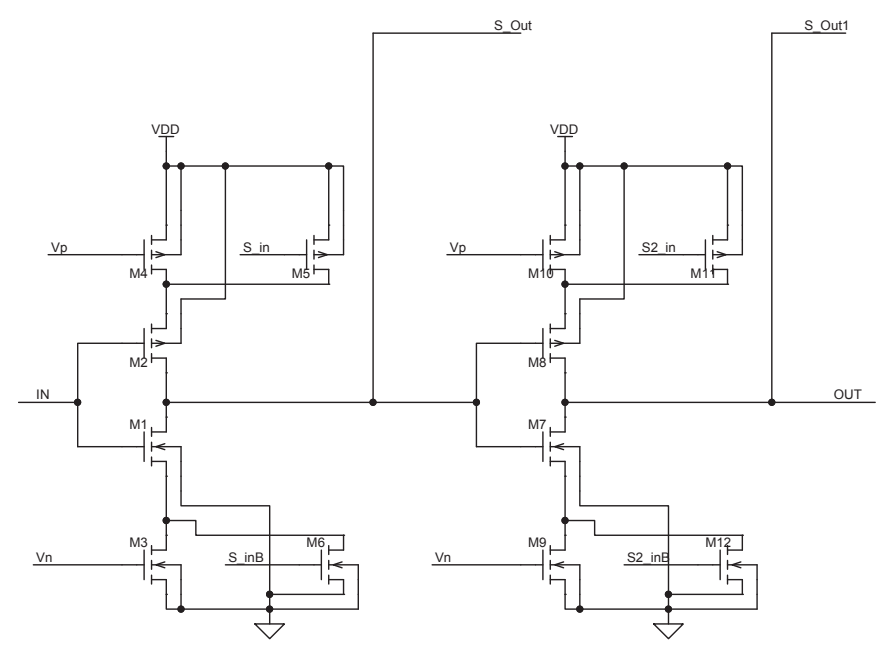

Figure 3.12: hows the architecture of a dual inverter based delay cell. The transistors M4, M5,M3 and M6 are used for starving the current through the inverter chain [43].

Transistors M4,M5, M10 and M11 are PMOS and M3,M6,M9,M12 are NFETS which are used for starving current through the inverter by the signals vp and vn as shown in figure 3.12 . The first signal S_in is selected the output delay is a combination of first inverter and the second that is current starved as is low, hence obtaining one set of delay. As S_inB is selected the first inverter is current starved and the second is not, thus obtaining a second delay. 


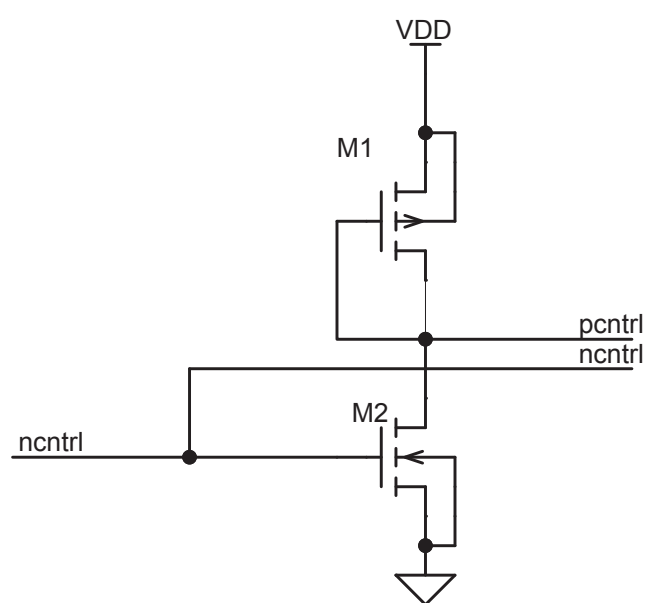

Figure 3.13: The current mirror consists of two transistors M1 and M2. When M2 is turned on the M1 also turns on hence providing the bias to the delay cell [43].

Figure 3.13 shows a current mirror which is tunable. The value of the bias is changed with "nctrl" voltage. The same current bias circuit is used to control all the voltages for the delay cell which are connected to vn and vp as shown in figure 3.12. The architecture is shown in figure 3.14 .

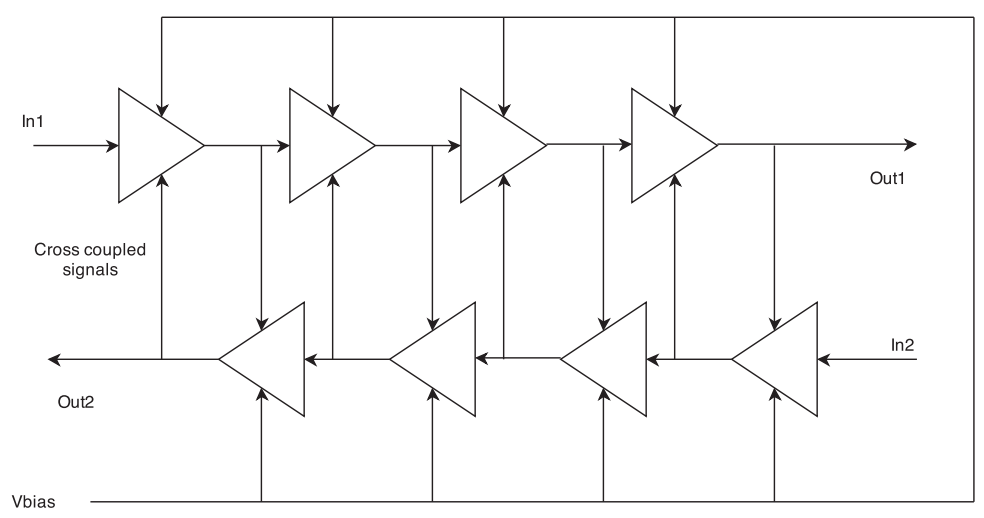

Figure 3.14: The cross coupled chains are shown, with the input and output connections. In addition the Vcntrl signal is applied to the cirucit shown in 3.13, this enables to tune the delay stages due to current starving technique [43]. 


\section{Variable delay Cell Using Varactors}

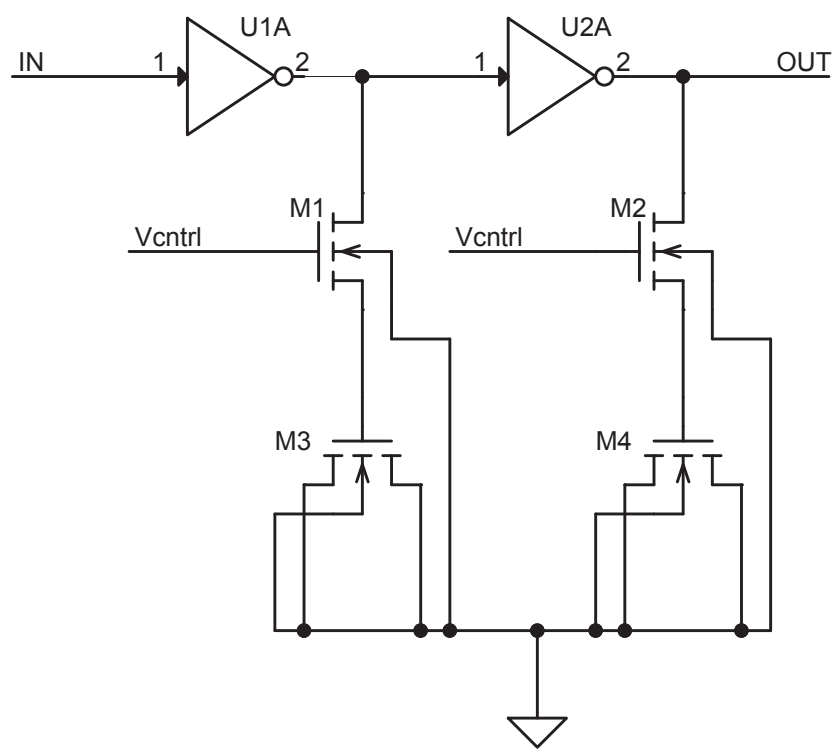

Figure 3.15: A tunable delay cell is shown here, M1 and M2 transistors are used as current starving circuits. M3 and M4 are connected in a way where they are realized as MOSCAPS [44].

$M 1$ and $M 2$ are controlled by a voltage $V_{\text {cntrl }}$ which limits the MOSCAPS $M 7$ and $M 8$ to charge or discharge slowly hence decreasing the slew rate. The voltage applied at the gate of the MOSCAP is varied and the capacitance changes as $V_{G S}$ changes. The effective capacitance reduces as the $V_{G S}$ is decreased which lowers the slew rate. The slew rate is a function of time difference gain. This helps in improving the gain of the system. 


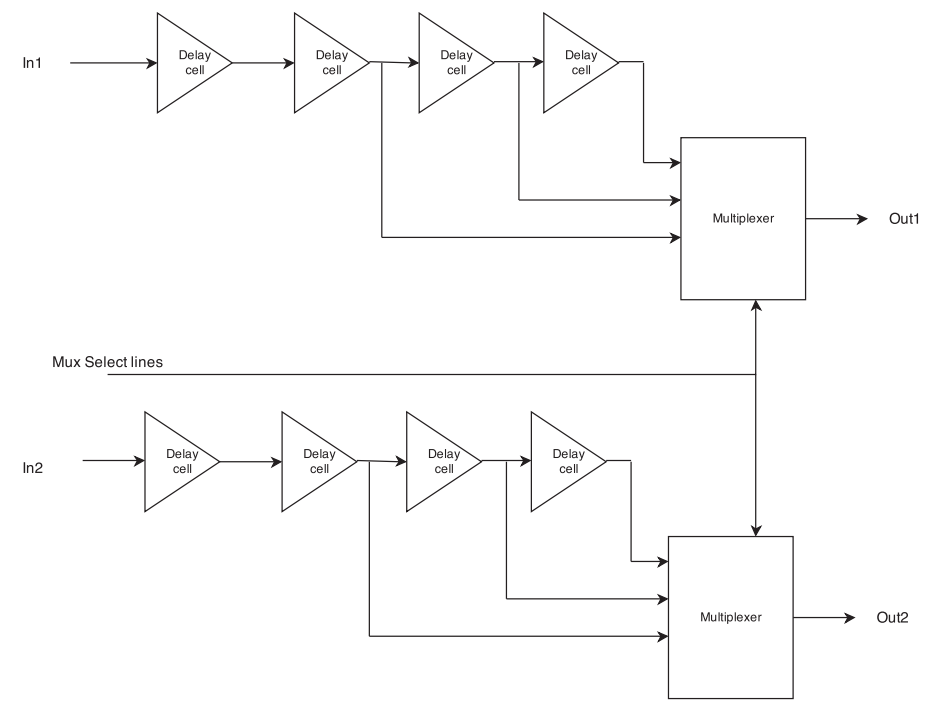

Figure 3.16: Figure shows a selectable output delay chain. The select lines can tap different delay outputs from the delay lines [16].

The multiplexers select the different delay output stages and can be selected by using $(N-1)$ combinations. Thus making the gain digitally selectable shown as,

$$
\Delta T_{\text {Out }}=(N-1) \cdot \Delta T_{\mathrm{In}}
$$

where $\Delta T_{\text {Out }}$ is the output delay, $\Delta \cdot T_{\text {In }}$ is the input time difference and $N$ is the number of stages. This equation relates the gain with the the input time difference [16]. 


\subsubsection{Time Difference Amplifier using Differential signalling}

The previous section was describing the different architectures of a time difference amplifier which have a single ended input and output. This section will describe time difference amplifiers that use differential signalling. The differential signalling is popular due to better rejection of noise and increased differential gain. Differential signalling uses two complementary signals over a common mode input voltage. Devices that use differential signalling are USB, HDMI, LVDS.

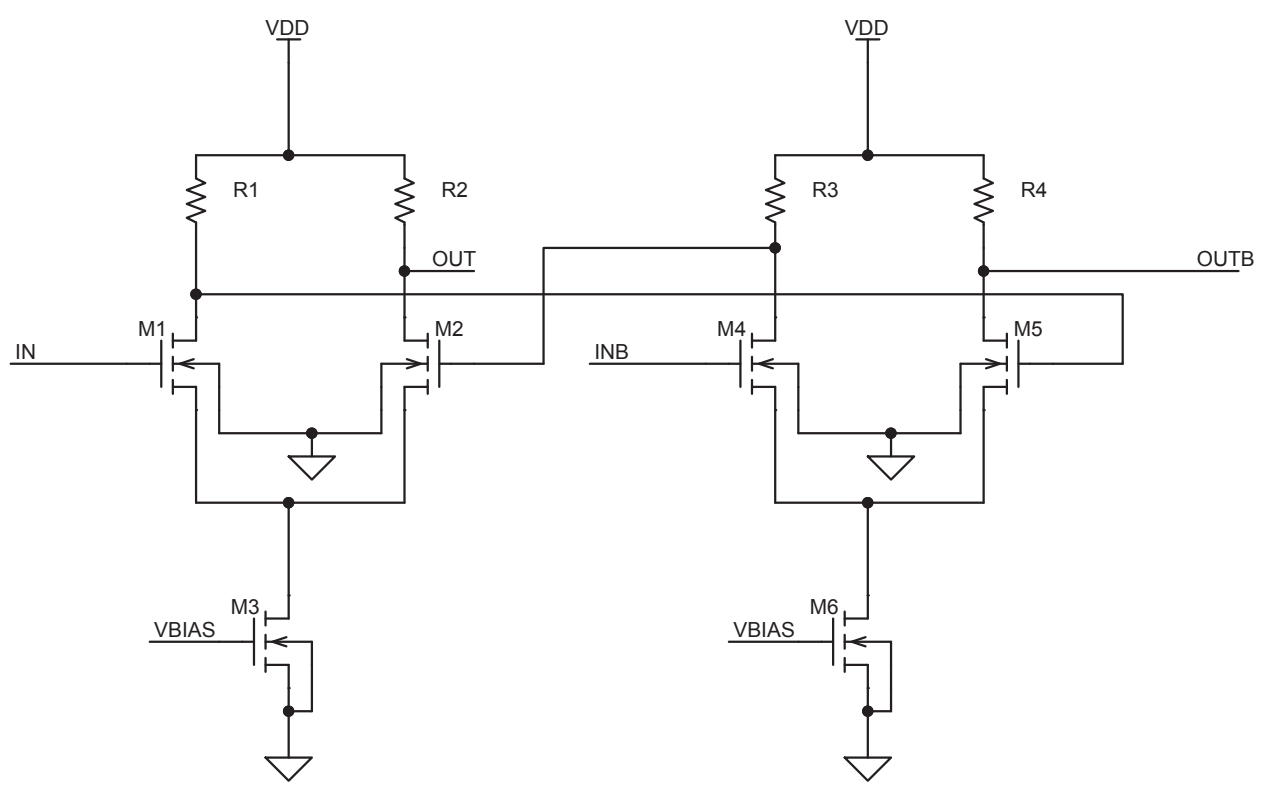

Figure 3.17: Transsitors M1 and M2 form a differential pair, similarly M4 and M5 are also differential pairs. M3 and M6 are used for setting the bias current through the branches [44]

Figure 3.17 shows a differential signalling based time difference amplifier. The resistors are loads or pull ups, and the cross coupled nmos transistor logic transfers charge from one branch to another. When the first pulse is seen at $M 1$ the current in the second branch is cut off, as the second pulse arrives after $\Delta t$ the transistor 
M3 turns on and changes the current in the first branch. The output of the time difference circuit is fed to a comparator that conditions the signal output, and the whole circuit behaves a time difference amplifier.

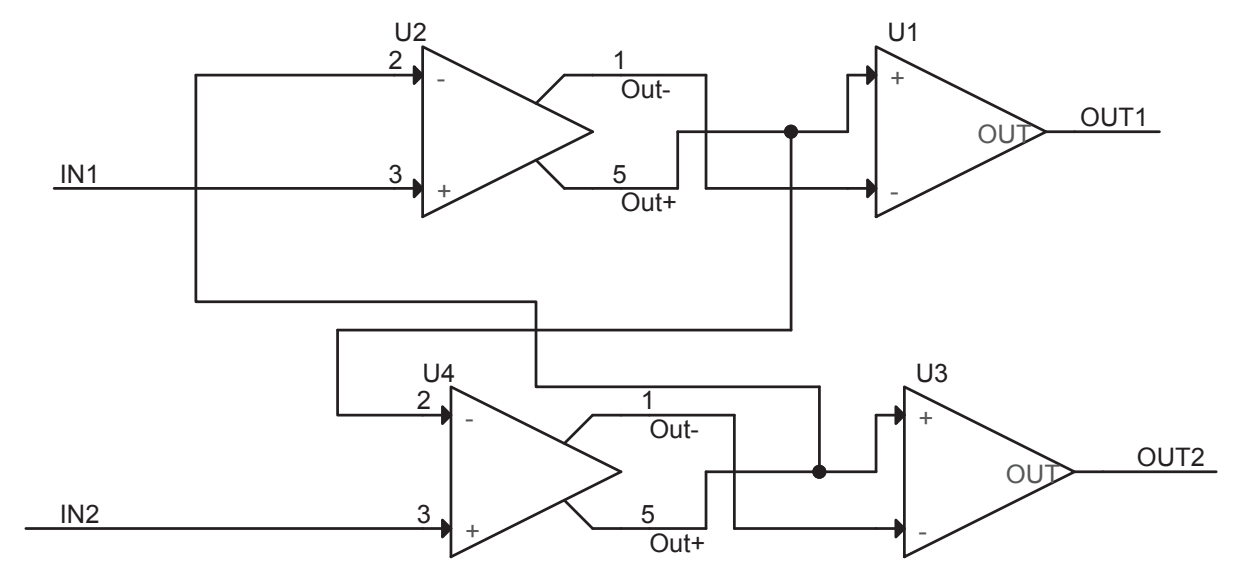

Figure 3.18: Opamps U2 and U4 have differential outputs, Opamps U1 and U3 are used as comparators, which the compares the output of the fully differential stage. The outputs are fed back to the inputs. Out1 and Out2 provide a output time difference. The circuit also acts as an arbiter [32]

The time bandwidth of the amplifier [32] is,

$$
T_{\mathrm{BW}}=T_{\mathrm{r}} \cdot\left(\frac{G_{\mathrm{t}=0}}{\sqrt{2}}\right)
$$

where, $T_{\mathrm{r}}$ is the rise time of the signal in second, $G_{\mathrm{t}=0}$ is the gain at $t=0$. The time bandwidth is used as a performance metric factor for the time difference amplifiers. 


\subsubsection{Variable delay cell using differential logic}

This section describes an implementation that uses the concept of the variable cell delay. The same concept of current starving is used to control the delay. The logic style is differential which increases the linearity compared to the single ended signalling based time difference amplifiers as shown in figure 3.19 .

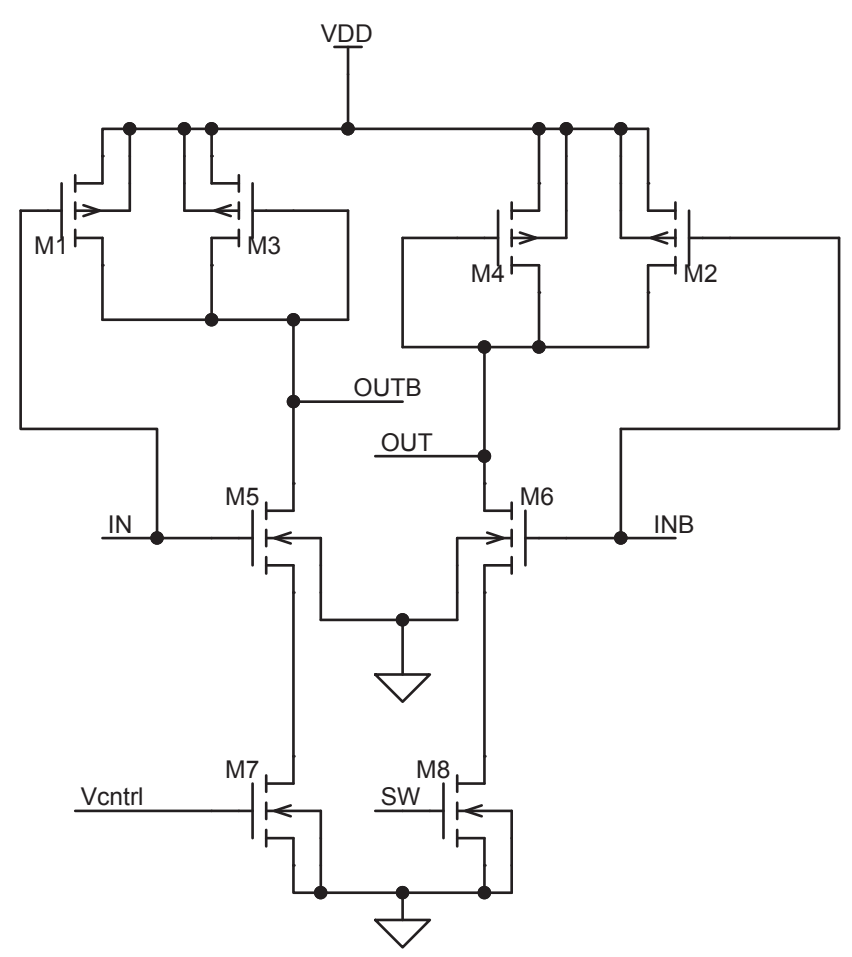

Figure 3.19: Transistors M1 through M4 are connected as active loads. M3 and M4 are cross coupled to increase the differential gain. M5 and M6 are the differential pair. M7 is used for current starving, and M8 is connected to the cross coupled pair, the output switches from the short delay to long delay [45] .

The vernier chain delay is implemented using differential delay blocks and the time difference amplifier is realized. 


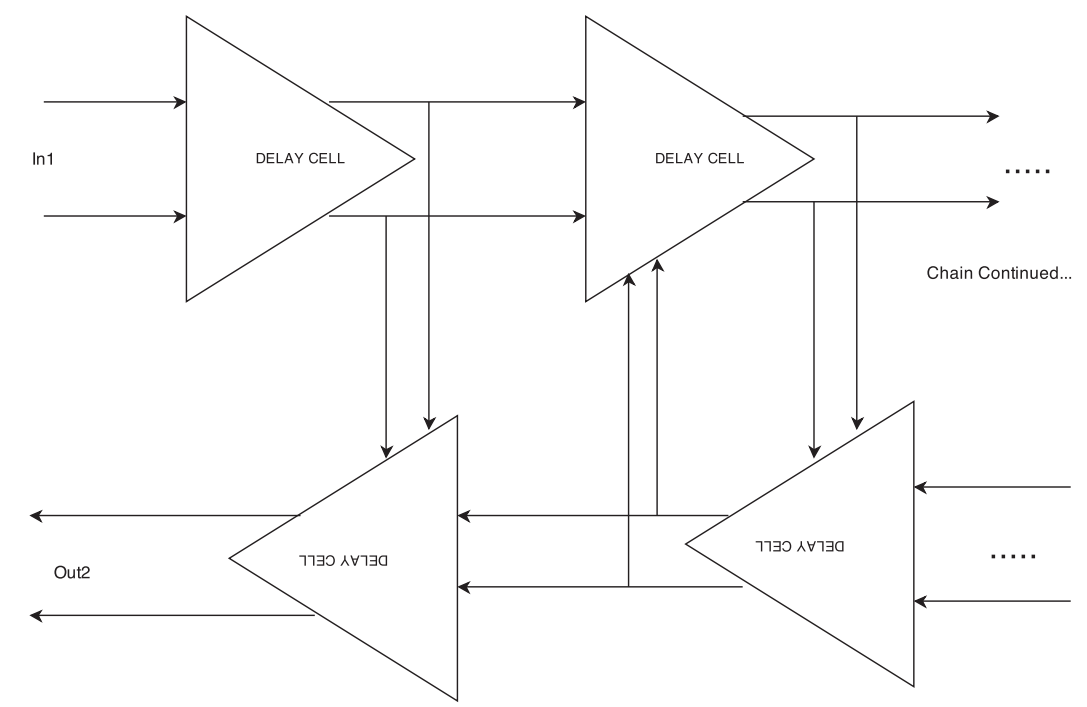

Figure 3.20: Figure 3.20 shows a portion of the delay chain based architecture with differential signalling techniques $[17,45,46]$.

Figure 3.20 shows the delay chain based architecture with differential signalling techniques [46], [17]. The linearity is better compared to the single ended architecture. This chapter describes various implementations that are done in making a time difference amplifier circuit. The below table gives a summary of the implementations described in this literature review and compared to this work. The design specifications and implementation are mentioned in the next chapter for this work. 


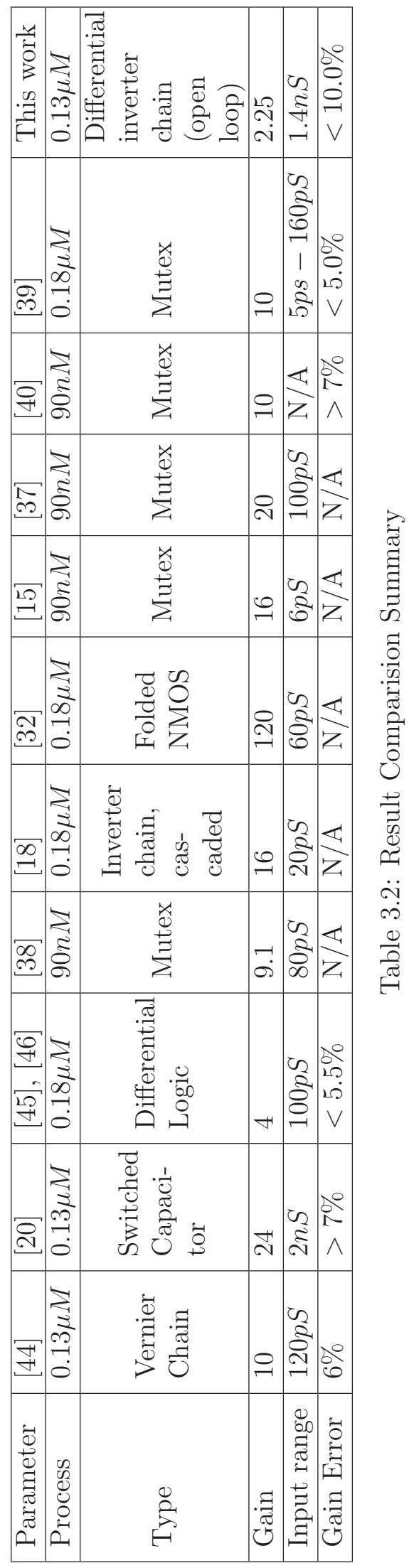




\section{Chapter 4}

\section{Implementation}

The implementation of the TDA, its simulation results and the design methodology are described in this chapter.

\subsection{Design Specifications}

This chip is intended for use in a florescence experiment setup at University of Massachusetts , Amherst U.S.A. The specifications of the device listed below were provided by Dr.Christopher Salthouse (UMASS).

Open loop Gain $\geq 2$

Input Range, $0<T_{\text {in }} \leq 1 n S$

Open loop Gain error , <10\% 


\subsection{Switched Inverter chain based approach}

Out of the several architectures for TDAs discussed earlier a chain of inverters that can switch between a short and long delay has been selected for this implementation. In this scheme each inverter can switch from a short to a long delay. Current starving is also used to achieve the required delays. The implementation can be based on either single ended or differential signaling. The schematic for the device that uses single ended signaling is shown in Figure 4.2 and its system level design in figure 4.3. Figure 4.1 shows a hierarchical symbol.

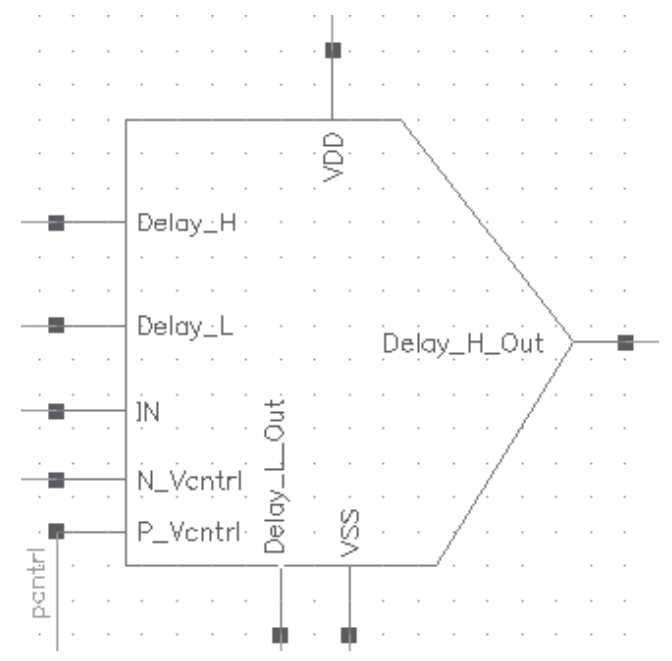

Figure 4.1: Figure shows the symbol of a single ended input inverter symbol. An hierarchical design approach is taken in this design 


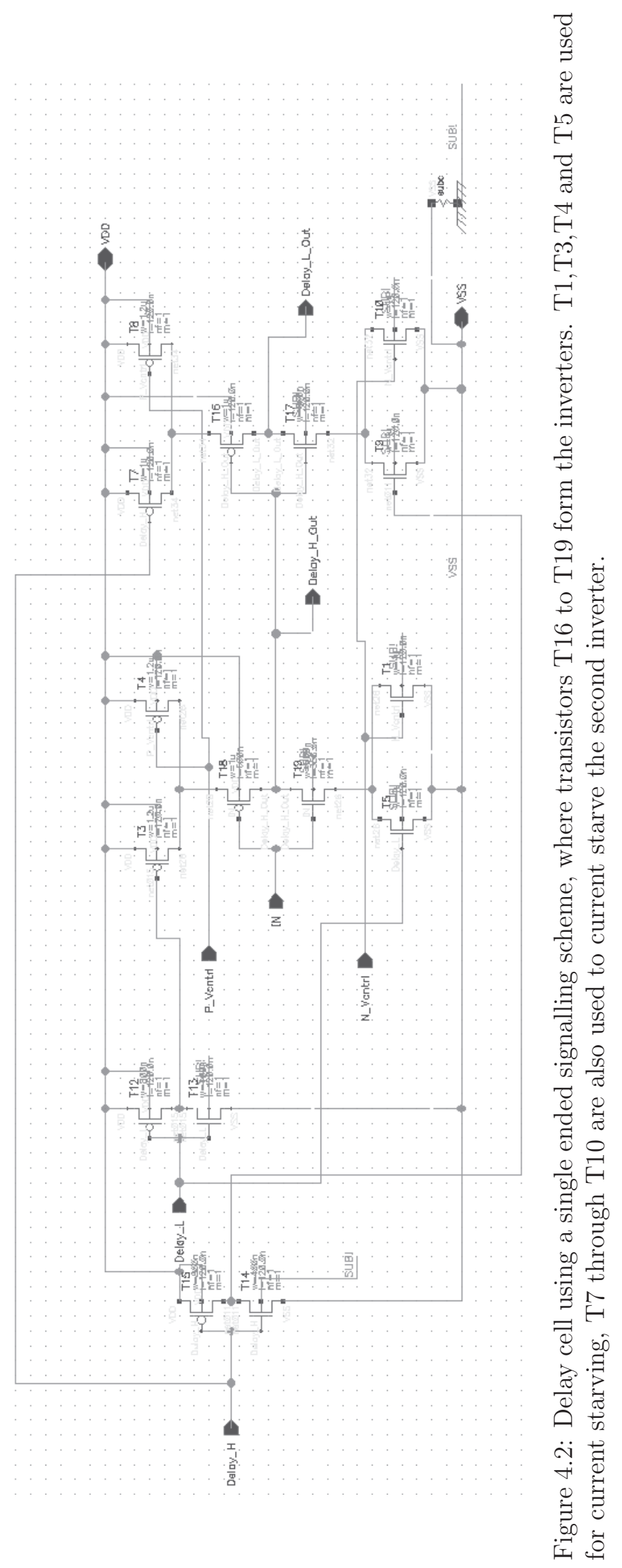




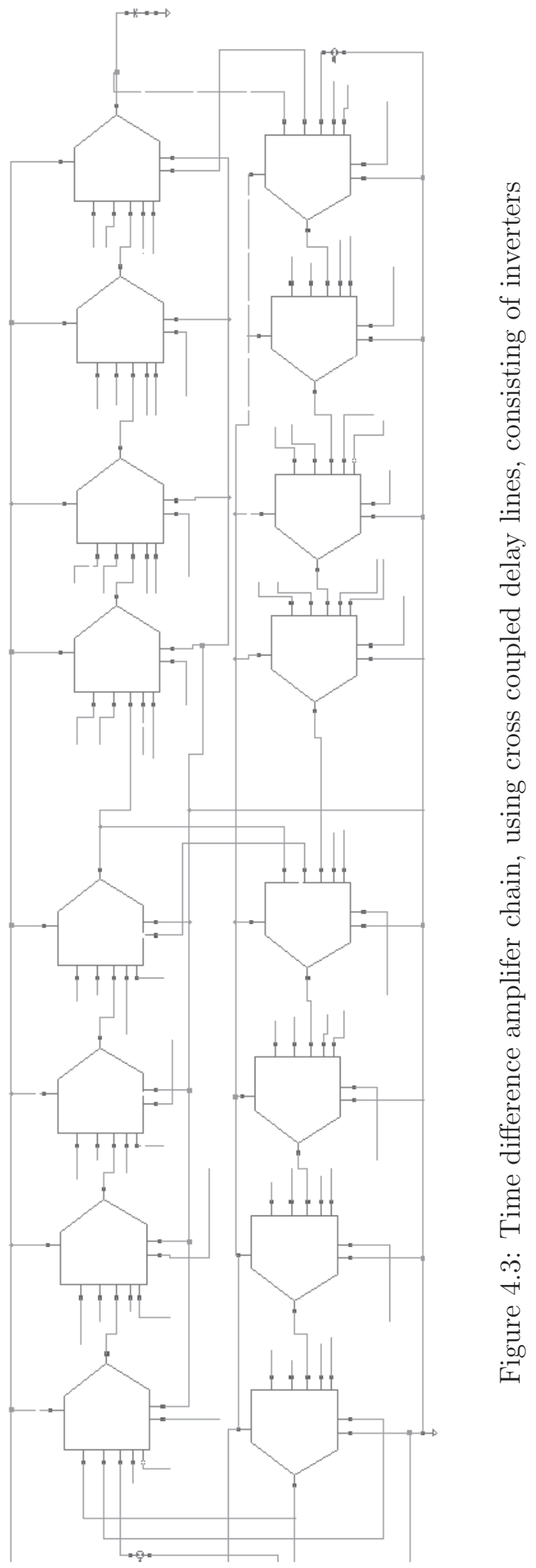




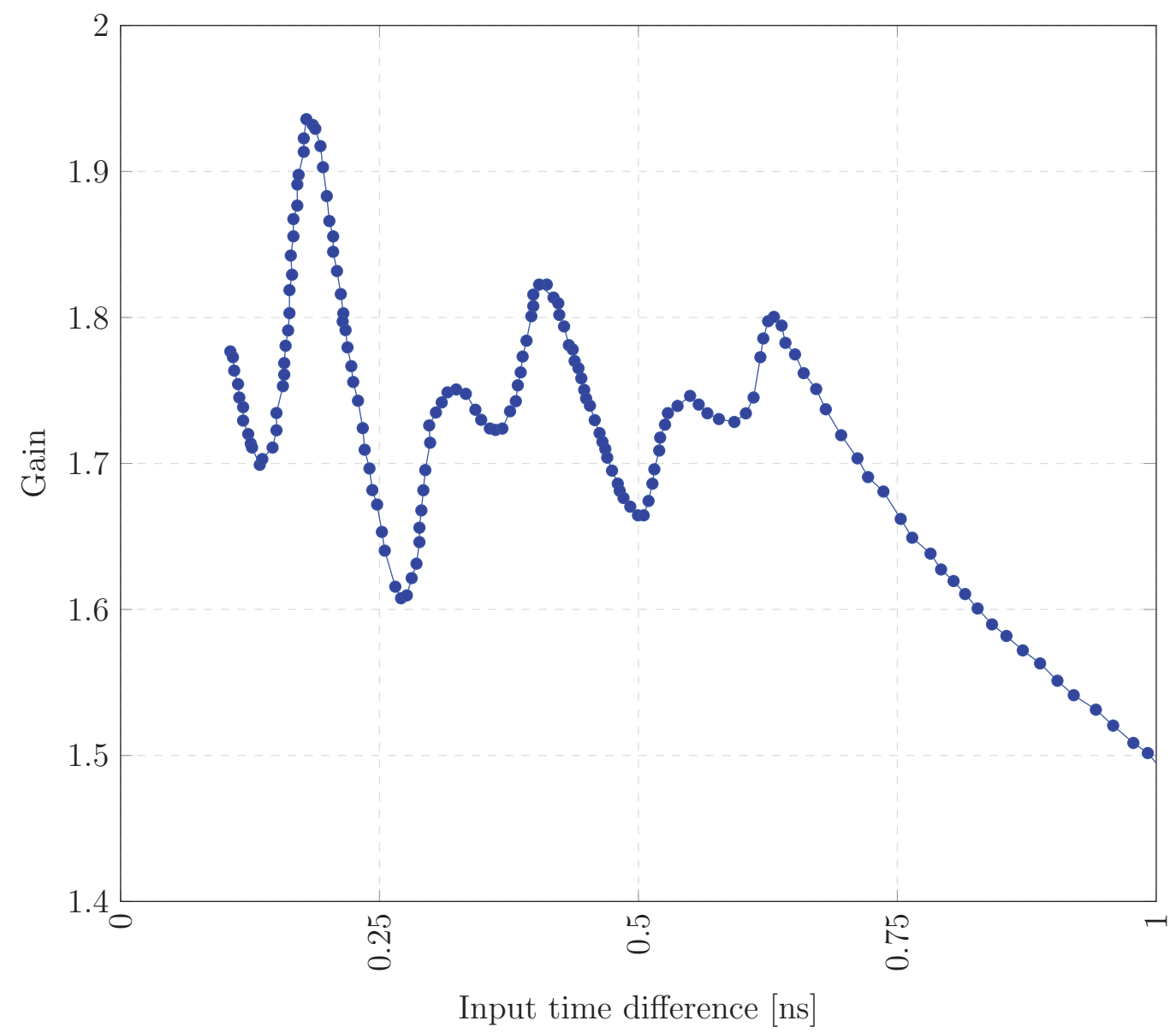

Figure 4.4: Sample plot show the gain $\mathrm{v} / \mathrm{s}$ the input time difference, one can see the variations of the gain after simulating on cadence tool.

As the single ended design approach has poor linearity as indicated by the transfer function and gain plot in figure above, the chip was implemented using differential signaling scheme.

The reason for the steps in the gain plot are due to the switching action between the short delay to the long delay. The number of stages also play a role in determining the linearity. Larger the number of stages better the linearity. 


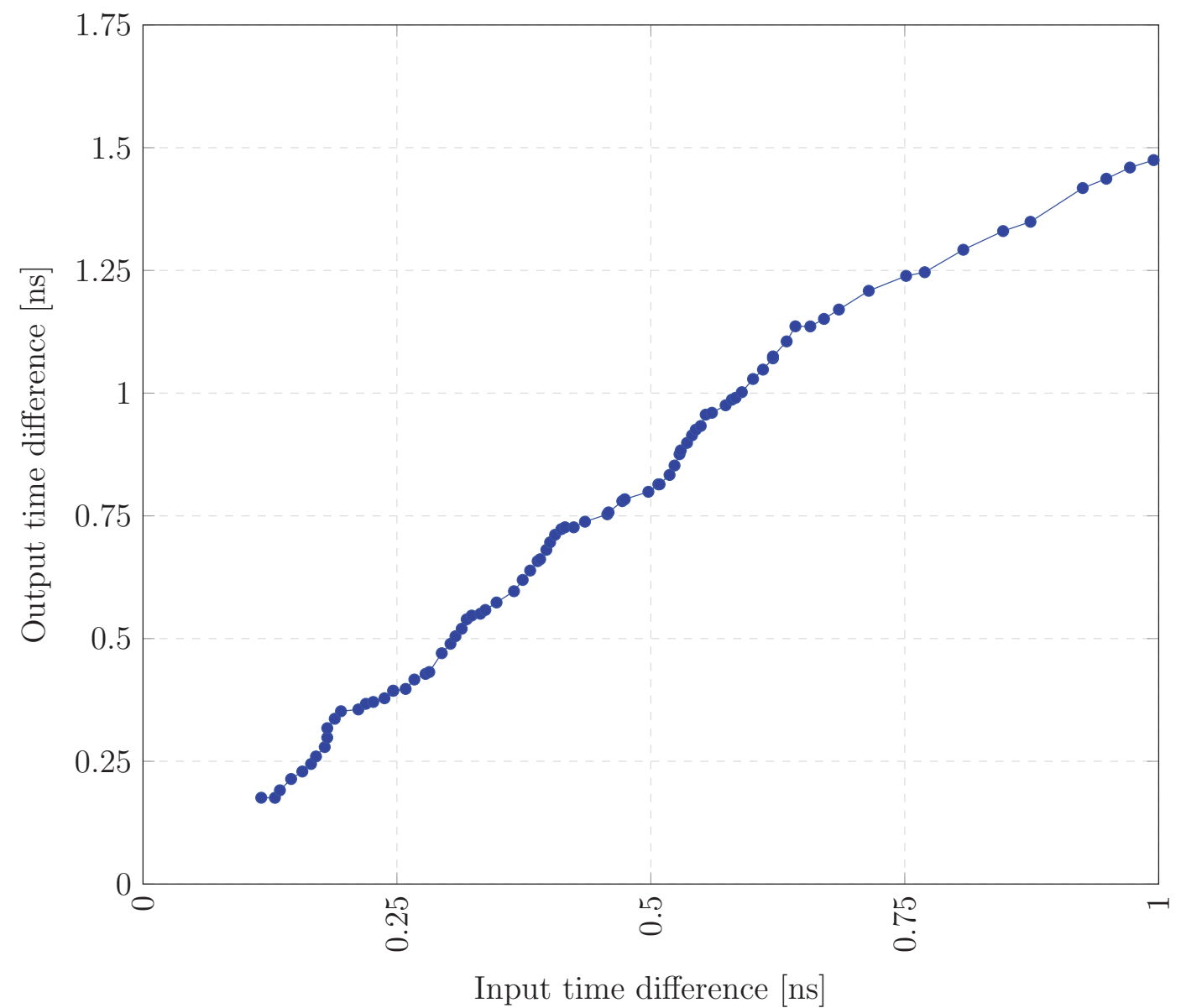

Figure 4.5: Sample plot show the output time difference $\mathrm{v} / \mathrm{s}$ the input time difference after simulating the single ended architecture. 


\subsection{Differential signalling based TDA}

\subsubsection{Architecture}

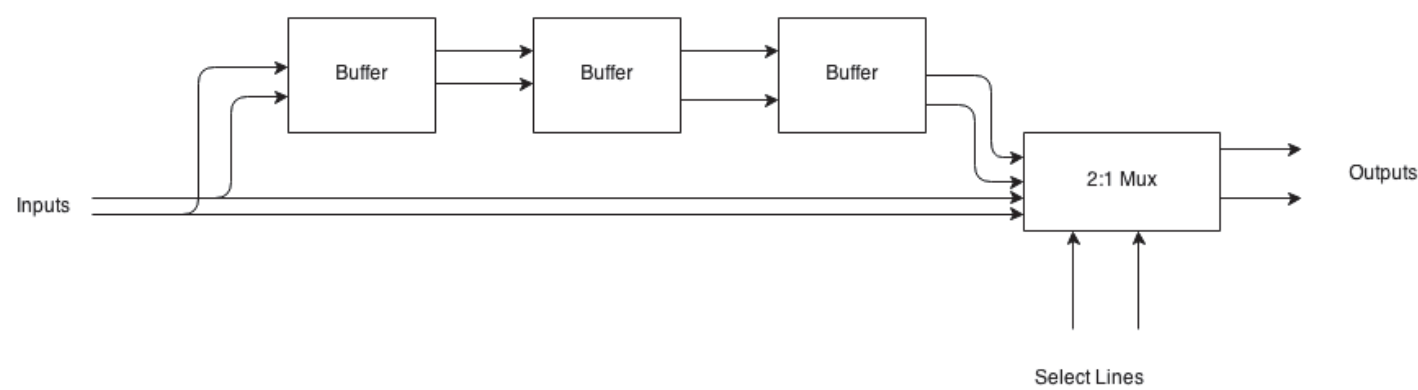

Figure 4.6: The block diagram depicts the architecture of the new time difference amplifier. The delay cell is made up of three buffers and an 2:1 multiplexer,.

The inverter with switchable delay is the heart of this design. The basic delay cell is shown in figure 4.6. The longer delay required is realized by cascading multiple buffer stages. A multiplexer is used to select either the input signal itself or the output of buffer stages. The multiplexer select line is used to select between a short and a long delay. The propagation delay of the multiplexer constitutes the shorter delay when the select line is a logic low, while the longer value is the delay provided by the buffer chain and propagation delay of the multiplexer when the select line is high.

$$
T_{\text {pd Short }}=T_{\text {pd Mux }}
$$

gives the propagation delay of the shorter chain which is equal to the propagation delay of the multiplexer. The longer delay is,

$$
T_{\text {long }}=\left(N \cdot T_{\text {buffer }}\right)+T_{\text {mux }}
$$


Where $N$ represents the number of buffer stages per cell, $T_{\text {long }}$ is the propagation delay the longer chain, $T_{\text {buffer }}$ the propagation delay of the buffer alone, and $T_{\text {mux }}$ gixes the propagation delay of the multiplexer.

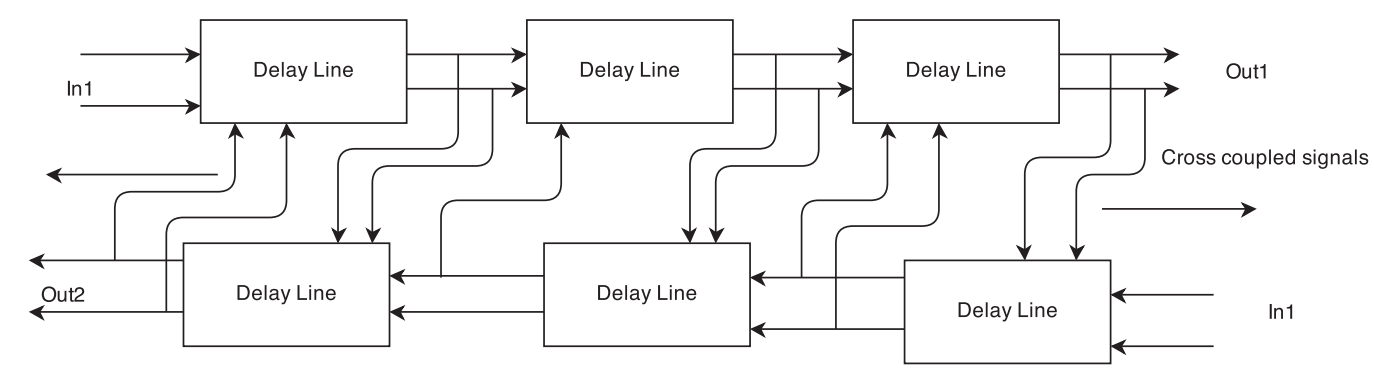

Figure 4.7: The delay lines are connected as a chain and the outputs are cross coupled to the adjacent chain.

As seen in figure 4.7 the TDA is formed by cascading several delay cells. Two such chains are formed and the chains are cross coupled such that the select signal of a delay cell in one chain is from corresponding output stage of the other chain. The inputs and outputs of the buffer chains are the input and output signals of the device. As the signal propagates through the chain the select signal of the element in the adjacent chain are switched to a logic ' 1 ' and making it delay the signal through it if it had not already passed this stage. When the signals in the two chains cross over, the product of the number of cells that both the signals have to traverse thereafter and the longer delay provided by the delay elements represent the amplified time difference between the output signals and is,

$$
\Delta T_{\text {Out }}=T_{\text {out1 }}-T_{\text {out } 2}
$$

and the input time difference is,

$$
\Delta T_{\mathrm{In}}=T_{\mathrm{in} 1}-T_{\mathrm{in} 2}
$$




$$
G=\frac{T_{\text {out }}}{T_{\text {In }}}
$$

where $G$ is the gain of the amplifier.

The number of stages play an important role in determining the gain, while Figure 4.7 shows only three stages, the actual implementation has ten stages in each chain to achieve the required gain.

\section{Basic Cell Design}

Each delay cell has two basic components within itself, a set of inverters and a 2:1 multiplexer. Each such component in turn has sub blocks depicted in figure 4.8.

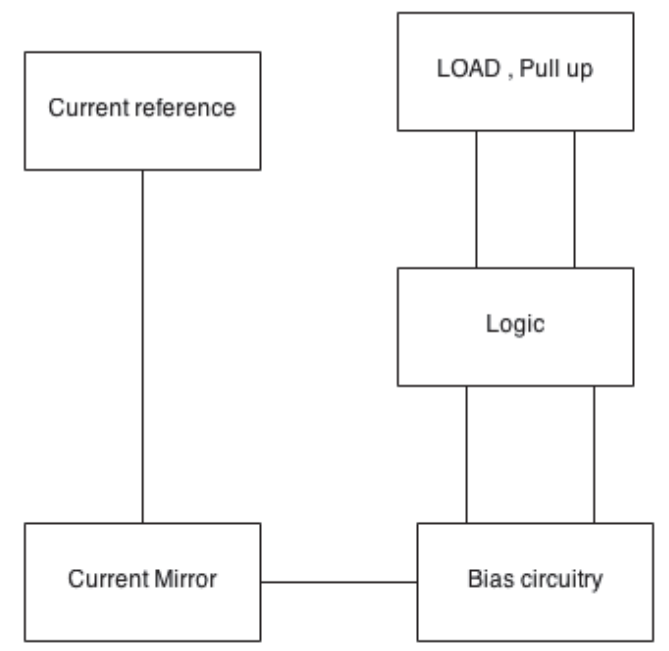

Figure 4.8: Buffer Block Diagram

The logic sub block decides the functionality of the cell and is either an inverter or 2:1 multiplexer. The bias block sets up current through the branches of the logic block. The output of the logic block needs load resistors that are implemented as part of the Load/pull up block. The bias circuitry is activated by the current mirror fed by an off chip reference. The detailed internal design with respect to an inverter or a mux cell follows in the subsequent sections. 


\subsubsection{Inverter Design}

The Hierarchical symbol of the inverter is shown in figure 4.9 followed by the pin descriptions for this block.

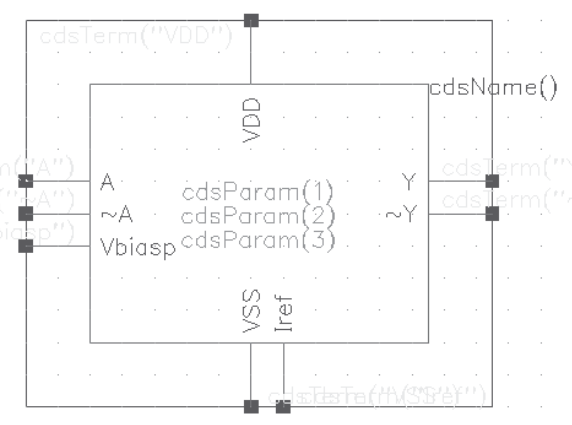

Figure 4.9: Inverter Hierarchical symbol

\begin{tabular}{|c||c|c|}
\hline Pin & Type & Pin Description \\
\hline A & I & Differential Input 1 \\
\hline A & I & Differential Input 2 \\
\hline Y & O & Differential Output 2 \\
\hline Y & O & Differential Output 2 \\
\hline Vbiasp & P & Active load bias \\
\hline Iref & P & Current reference pin \\
\hline VDD & P & Supply Power Pin 1.2V \\
\hline VSS & P & Supply Ground Pin \\
\hline
\end{tabular}

Table 4.1: Inverter Pin descriptions 


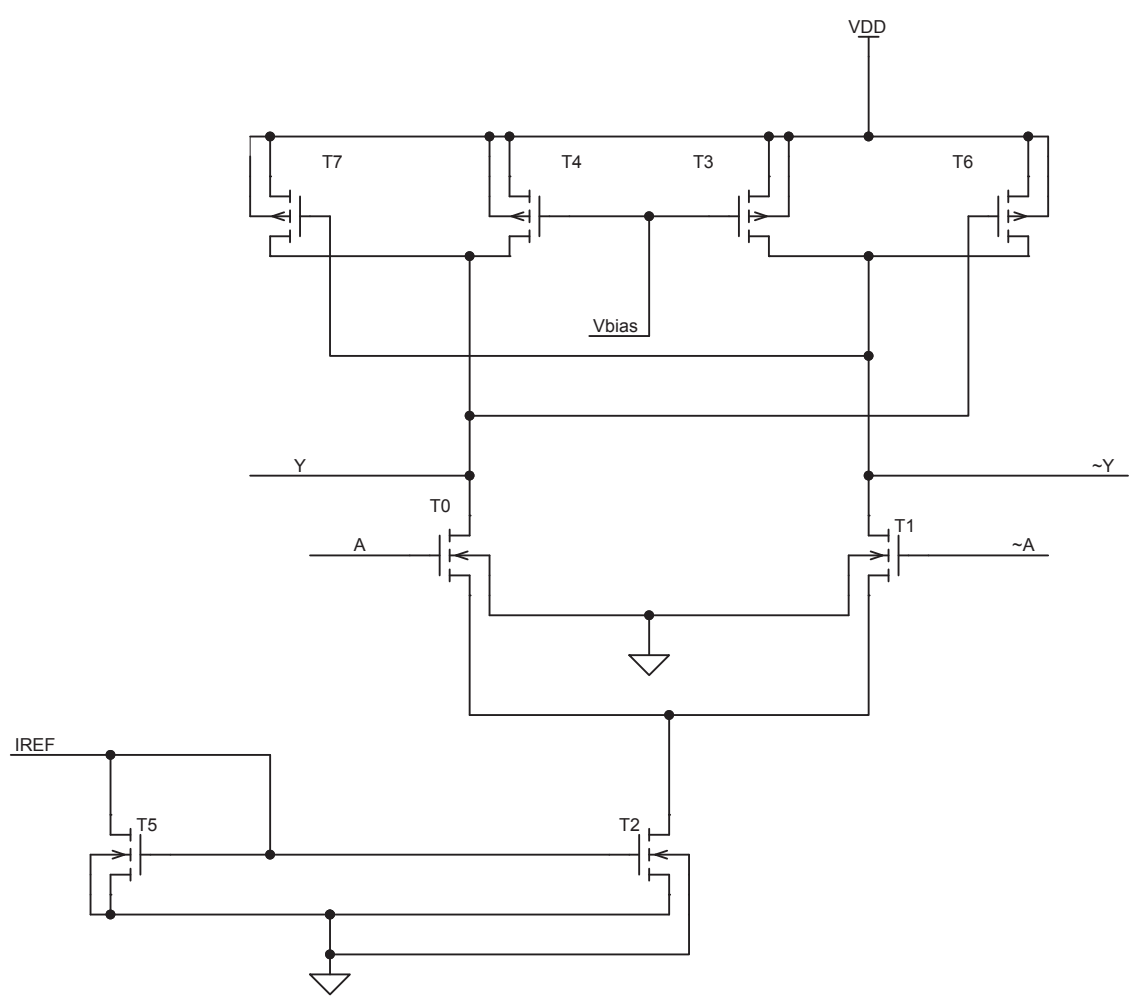

Figure 4.10: Inverter Schematics

Schematics of the inverter cell as on figure 4.10. T0 and T1 form the logic block, in this case an inverter. Loads are normally passive resistors but these would occupy a large amount of die space. In this design to minimize die space, T3, T4, T7, T6 which are ' $\mathrm{P}$ ' channel MOSFETs form the active loads/pull ups and replace the bulky resistors. The sizes of these transistors are selected such that they will operate in the triode region. Their gates are driven with a off chip bias voltage that is greater than $0 \mathrm{~V}$ but made the threshold voltage is maintained. $\mathrm{T} 2$ and $\mathrm{T} 5$ are part of the bias block. The operation was fairly simple, the external reference current is mirrored onto the differential pair in the logic block, and sets up branch currents through T0 and T1. When a differential input is applied to the logic block, current is steered between the left or right arm based on the input 
signal and the output nodes are either pulled high or low based on the input signal.As this TDA design needs a non-inverting buffer stage and this basic logic block is an inverter, the outputs of one stage are cross coupled to the input of the next to realize a non inverting buffer. The transistors $\mathrm{T} 6$ and $\mathrm{T} 7$ that are in parallel with the loads are driven by the output of the other arm hence cross coupled. Use of T6, T7 in this mode results in increased differential gain of the block and therefore contributes to the overall gain of the TDA.The implementation described above, uses a design approach similar to the one mentioned in [47].

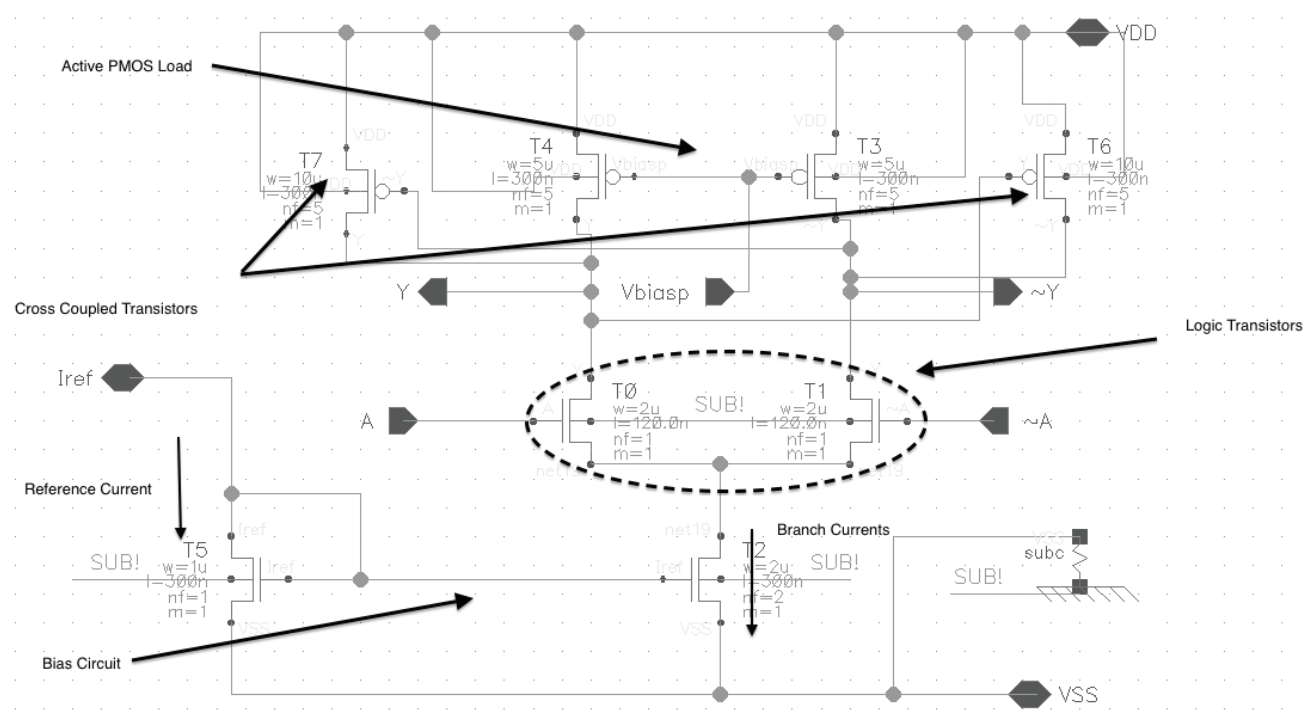

Figure 4.11: Inverter Schematics

Figure 4.11 identifies various blocks in the schematic and also indicate the reference and branch currents that flow the various transistors.

The aspect ratios of the of the various transistors used in this block are shown in tabular form in Table 4.2 


\begin{tabular}{|c||c|c|}
\hline Transistor & Width in $\mu M$ & Length in $n M$ \\
\hline T0 & 2 & 120 \\
\hline T1 & 2 & 120 \\
\hline T2 & 2 & 300 \\
\hline T3 & 5 & 300 \\
\hline T4 & 5 & 300 \\
\hline T5 & 1 & 300 \\
\hline T6 & 10 & 300 \\
\hline T7 & 10 & 300 \\
\hline
\end{tabular}

Table 4.2: Inverter design Transistor Aspect Ratios

The transistor ratios are chosen after iterative simulations. The lengths of the transistors in the logic block are made minimum lengths to increase their operational speed, where as those of the bias transistors are chosen 3 times the minimum length to minimize short channel effects. The layout of the inverter is shown in figure 4.12 .

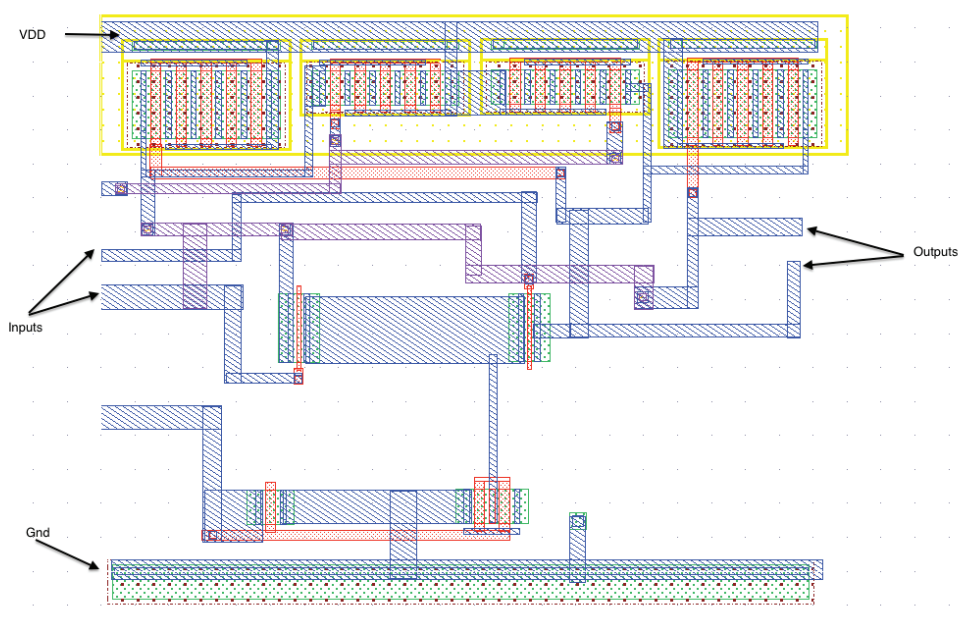

Figure 4.12: Inverter Layout 


\subsubsection{Multiplexer design}

This cell that is part of the delay cell is a 2:1 multiplexer and is used to delay the input signal by either a short delay or a long delay.The architecture is similar to the CML mux [48] with minimal changes. The hierarchical symbol of the mux block is shown in figure 4.13. Table 4.3 show the pin information for this cell.

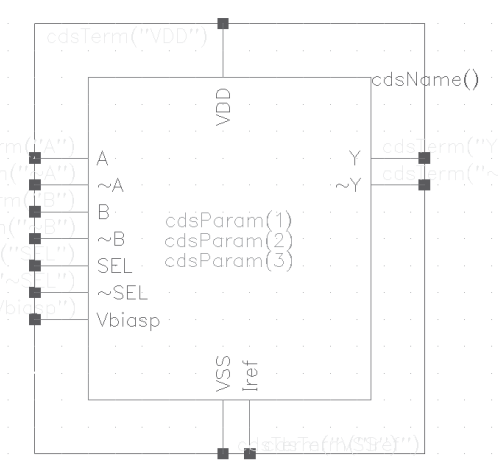

Figure 4.13: 2:1 Multiplexer Symbol

\begin{tabular}{|c||c|c|}
\hline Pin & Type & Pin Description \\
\hline A & I & Differential Input1 + \\
\hline A & I & Differential Input - \\
\hline B & I & Differential Input2 + \\
\hline B & I & Differential Input2 - \\
\hline SEL & I & Select line + \\
\hline SEL & I & Select line - \\
\hline Y & O & Differential Output + \\
\hline Y & O & Differential Output - \\
\hline Vbiasp & P & Active load bias \\
\hline Iref & P & Current reference pin \\
\hline VDD & P & Supply Power Pin 1.2V \\
\hline VSS & P & Supply Ground Pin \\
\hline
\end{tabular}

Table 4.3: 2:1 Mux Pin descriptions

The schematic diagram in figure 4.14 shows the modified 2:1 multiplexer. It has two differential inputs inputs $\mathrm{A}$ and $\mathrm{B}$ and based on the select signal the output follows A or B. A logic '1' at the select input select signal at input A. Please refer the truth table is in Table 4.4 .

The circuit schematic of this block is in figure 4.14. The logic block comprises of 


\begin{tabular}{|c|c|}
\hline Select input & $\mathrm{Y}$ \\
\hline 0 & $\mathrm{~B}$ \\
\hline 1 & $\mathrm{~A}$ \\
\hline
\end{tabular}

Table 4.4: 2:1 Multiplexer truth table

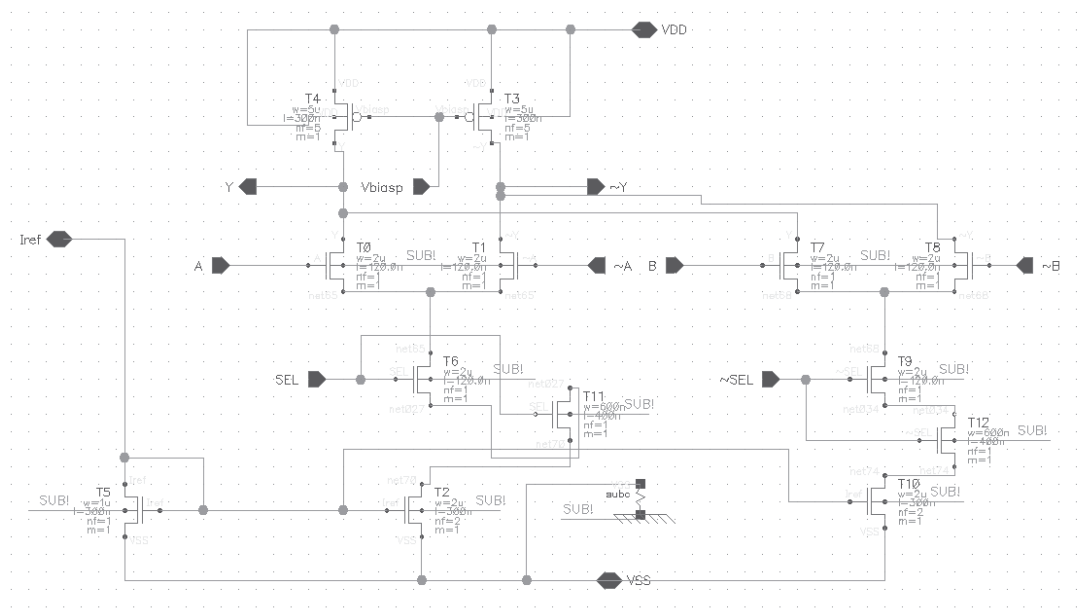

Figure 4.14: 2:1 Multiplexer Schematic

T0,T1,T7,T8 along with T6 and T9. Transistors T4 and T3 are part of the active load block.T0,T1 handle differential input A while T7,T8 handle input B. These share common active loads. Select transistors T6 and T9 decide which of these pairs will drive the active loads T3,T4 based on the select signal. An important addition to the circuit are the two transistors T11 and T12. These transistors are used to increase the threshold voltage of select transistors T6 and T9. Stacking of transistors in series increases the $V_{G S}$ value. The bulks of transistors T11,T12 are at GND potential and this introduces the "body effect". As the source terminal of the transistors are at a higher potential than the bulk $\left(V_{S B}>0\right)$ the overall threshold value increases. This body effect is found useful in this application and ensures that the select lines are properly selected to adjust to the output swings of individual delay lines. 


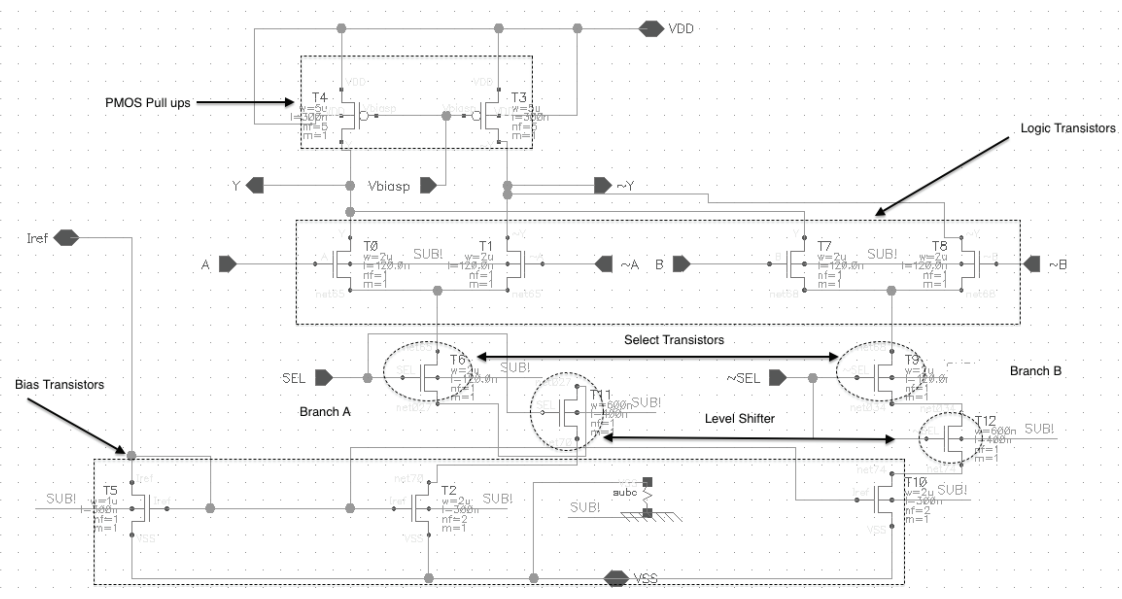

Figure 4.15: 2:1 Multiplexer Schematic Detail

Figure 4.15 identifies the various blocks of the cell on the schematic.Figure 4.16 shows the layout of the 2:1 Multiplexer and table 4.5 lists the aspect ratios of the various transistors in the block.

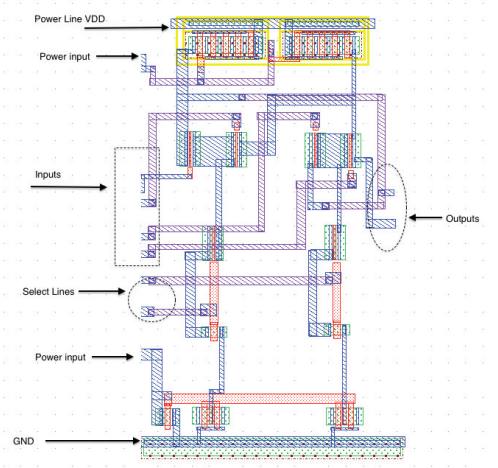

Figure 4.16: 2:1 Multiplexer Layout 


\begin{tabular}{|c||c|c|}
\hline Transistor & Width in $\mu M$ & Length in $n M$ \\
\hline T0 & 2 & 120 \\
\hline T1 & 2 & 120 \\
\hline T2 & 2 & 300 \\
\hline T3 & 5 & 300 \\
\hline T4 & 5 & 300 \\
\hline T5 & 1 & 300 \\
\hline T6 & 2 & 120 \\
\hline T7 & 2 & 120 \\
\hline T8 & 2 & 120 \\
\hline T9 & 2 & 120 \\
\hline T10 & 2 & 300 \\
\hline T11 & 0.6 & 400 \\
\hline T12 & 0.6 & 400 \\
\hline
\end{tabular}

Table 4.5: 2:1 Multiplexer design Transistor Aspect Ratios 


\subsubsection{Delay cell Implementation}

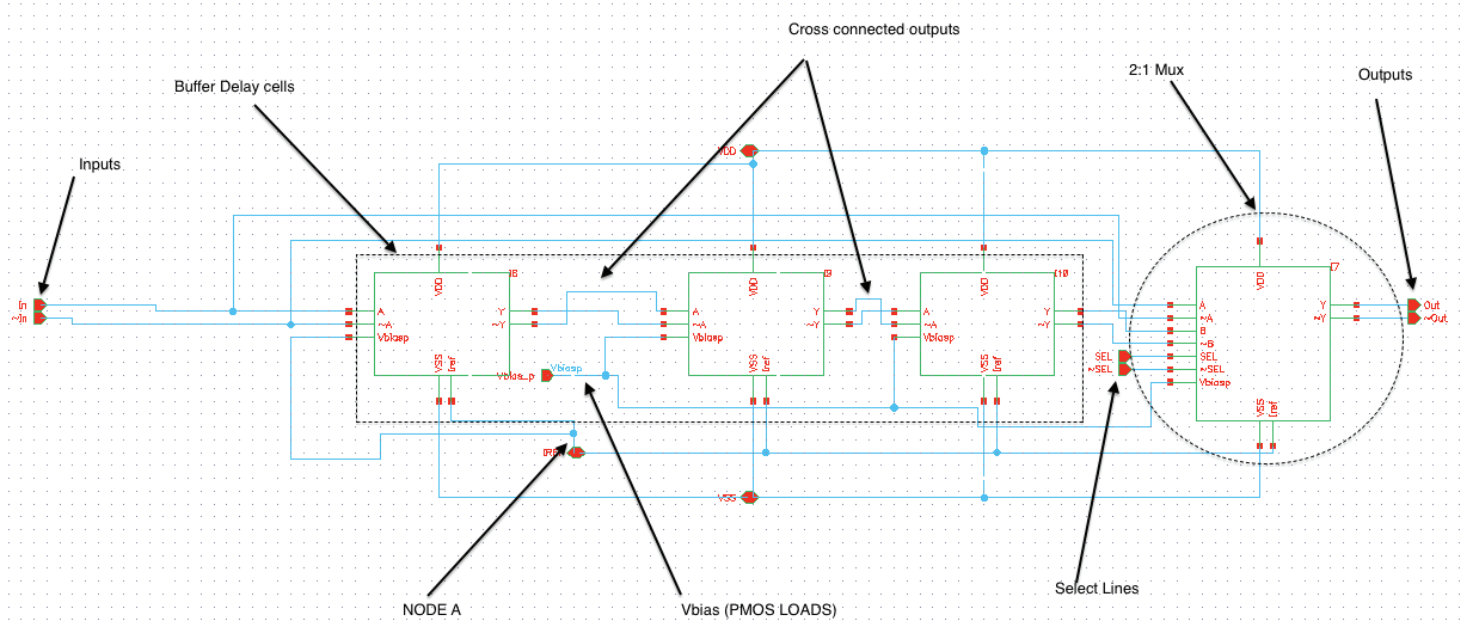

Figure 4.17: Delay Cell Implementation

The delay cell is the heart of this time difference amplifier design. The buffer and the multiplexer sub blocks described in detail above are used to build the delay cell. This cell is made up of three buffers and a multiplexer as seen in the schematic in figure 4.17. The inputs to the Delay Cell cell is subject to a short or a long delay based on the polarity of the select signal before it appears on the cell output. Apart from power supply pins, Iref and Vbias inputs supply the collective reference currents and bias voltages for the current mirrors and active loads respectively that are sub components within the inverter and multiplexer blocks. The dotted box in the schematic shows the inverter cells that are connected in series to realize a long delay. Note the cross connection between outputs of the previous stage and the inout of the next. This allows the use of the inverter cell as a buffer by preventing a phase reversal.Input $\mathrm{B}$ of the mux is connected to the delay blocks input and the input A of the mux to the output of the delay block. 
Therefore a logic ' 1 ' at the select input will delay the input signal to the block by a larger value.

The hierarchical symbol of the delay cell is shown in figure 4.18 and table 4.6 lists the pin information.

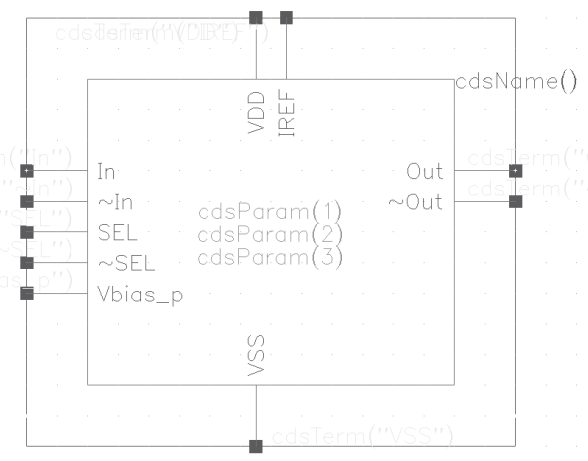

Figure 4.18: Delay Cell Implementation Symbol

\begin{tabular}{|c||c|c|}
\hline Pin & Type & Pin Description \\
\hline In & I & Differential Input1 + \\
\hline In & I & Differential Input1 - \\
\hline SEL & I & Select line + \\
\hline SEL & I & Select line - \\
\hline Out & O & Differential Output + \\
\hline Out & O & Differential Output - \\
\hline Vbiasp & P & Active load bias \\
\hline Iref & P & Current reference pin \\
\hline VDD & P & Supply Power Pin 1.2V \\
\hline VSS & P & Supply Ground Pin \\
\hline
\end{tabular}

Table 4.6: Delay cell pin descriptions

Layout of the delay cell is in figure 4.19 


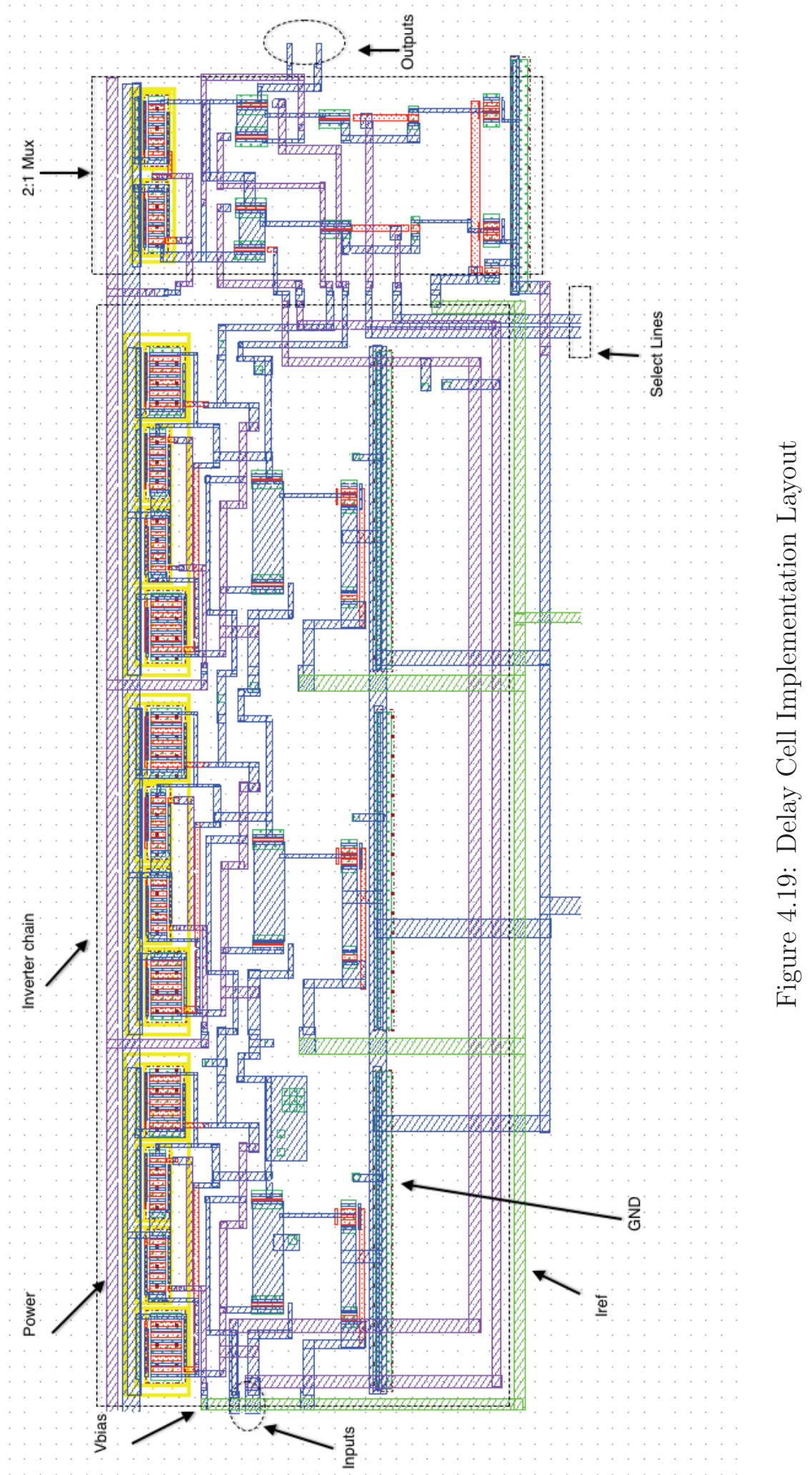




\subsubsection{Chip Level Implementation}

The time difference amplifier basically has two chains of cascaded delay Cells. Each chain comprises of ten delay cells. A part of the chain is shown in figure 4.20. The outputs of each cell in one chain is connected to the select lines of a counterpart element in the adjacent chain. As the signal propagate though the chains when the output of any delay cell changes from logic ' 0 ' to logic ' 1 ' the delay provided by the corresponding cell in the adjacent cell switch changes from a short delay to a longer delay, thus providing the gain. It should be noted that the output are cross connected to the select lines, to avoid inversion of the signal at output of the delay chain.

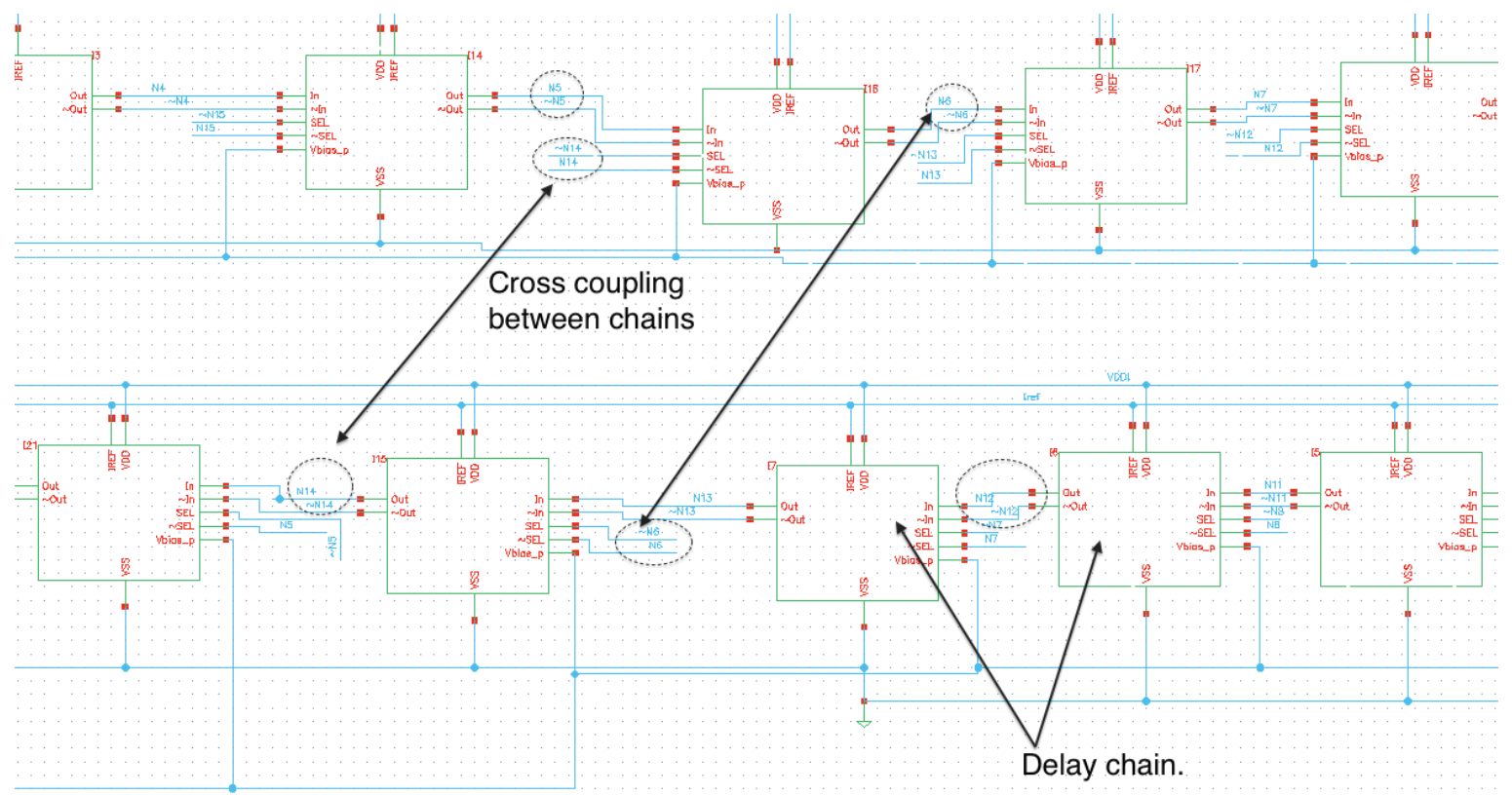

Figure 4.20: Time difference amplifier system implementation

The number of stages have been chosen as ten for this design keeping in mind the input range, linearity and the die size constraints. The number of stages determine the overall gain of the TDA. However after careful simulation it was 
observed that the linearity and the input range drops if the number of stages keep on increasing. Table 4.7 shows the pinning information.

\begin{tabular}{|c||c|c|c|}
\hline Name & Pin number & Pin Type & Description \\
\hline InB & 1 & I & Differential Input 1 \\
\hline In & 4 & I & Differential Input 1 \\
\hline In1 & 12 & I & Differential Input 2 \\
\hline In1B & 14 & I & Differential Input 2 \\
\hline Out & 7 & O & Differential Output 1 \\
\hline OutB & 9 & O & Differential Output 1 \\
\hline Out1 & 17 & $\mathrm{O}$ & Differential Output 2 \\
\hline Out1B & 19 & $\mathrm{O}$ & Differential input 1 \\
\hline VBIAS & 5 & $\mathrm{P}$ & Power \\
\hline Iref & 11 & $\mathrm{P}$ & Current reference input \\
\hline VDD & 15,16 & $\mathrm{P}$ & Power supply pin \\
\hline GND/VC & 18 & $\mathrm{P}$ & Power pin \\
\hline GND & 1,3 & $\mathrm{P}$ & Ground \\
\hline GND & 6,8 & $\mathrm{P}$ & Ground \\
\hline GND & $10,13,20$ & $\mathrm{P}$ & Ground \\
\hline
\end{tabular}

Table 4.7: TDA Chip Pin descriptions

$\mathrm{P}$ : Power pin, I : Input pin, O : Output pin. 


\subsection{Layout Implementation}

The two main considerations for the floor planning for this device are minimization of parasitic delays and easy routing. The two chains are laid out such that in one chain the signal travels from left to right and in the second chain which is a mirror image of the first chain, it propagates from the right to the left. This allows easy routing between the cells of the adjacent chains and also reduces the parasitic delay.

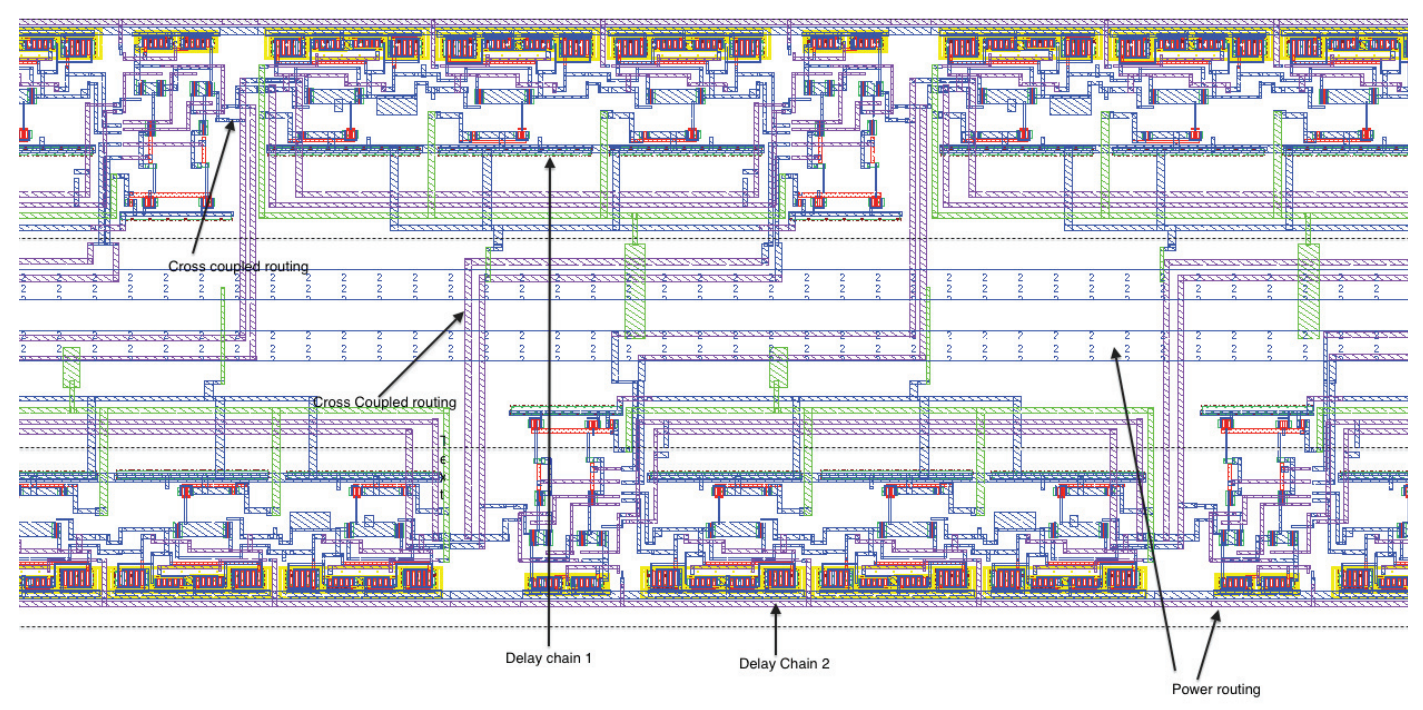

Figure 4.21: TDA layout

The integrated circuit has 20 bond pads that allow the device to be connected to the external world. The size of these bond pads were based on the specification of the lead bonder that would be used later. The pitch of these pads were selected to match the probing station that may need to be used at the time of testing the device. The bond pads were therefore chosen to be 95 micron square with a pitch of 150 microns. The total area estimated for the core circuitry and bondpads were approximately $1 \mathrm{~mm} \times 1 \mathrm{~mm}$ and the die size is chosen to the match the same 
value.The device has ESD diodes placed on all external connections to prevent damage from discharges at the time of handling. The full chip layout is shown in figure 4.22 along with Bondpads and ESD protection devices. Note that the signal pads are interleaved with GND pads to minimize cross talk. 


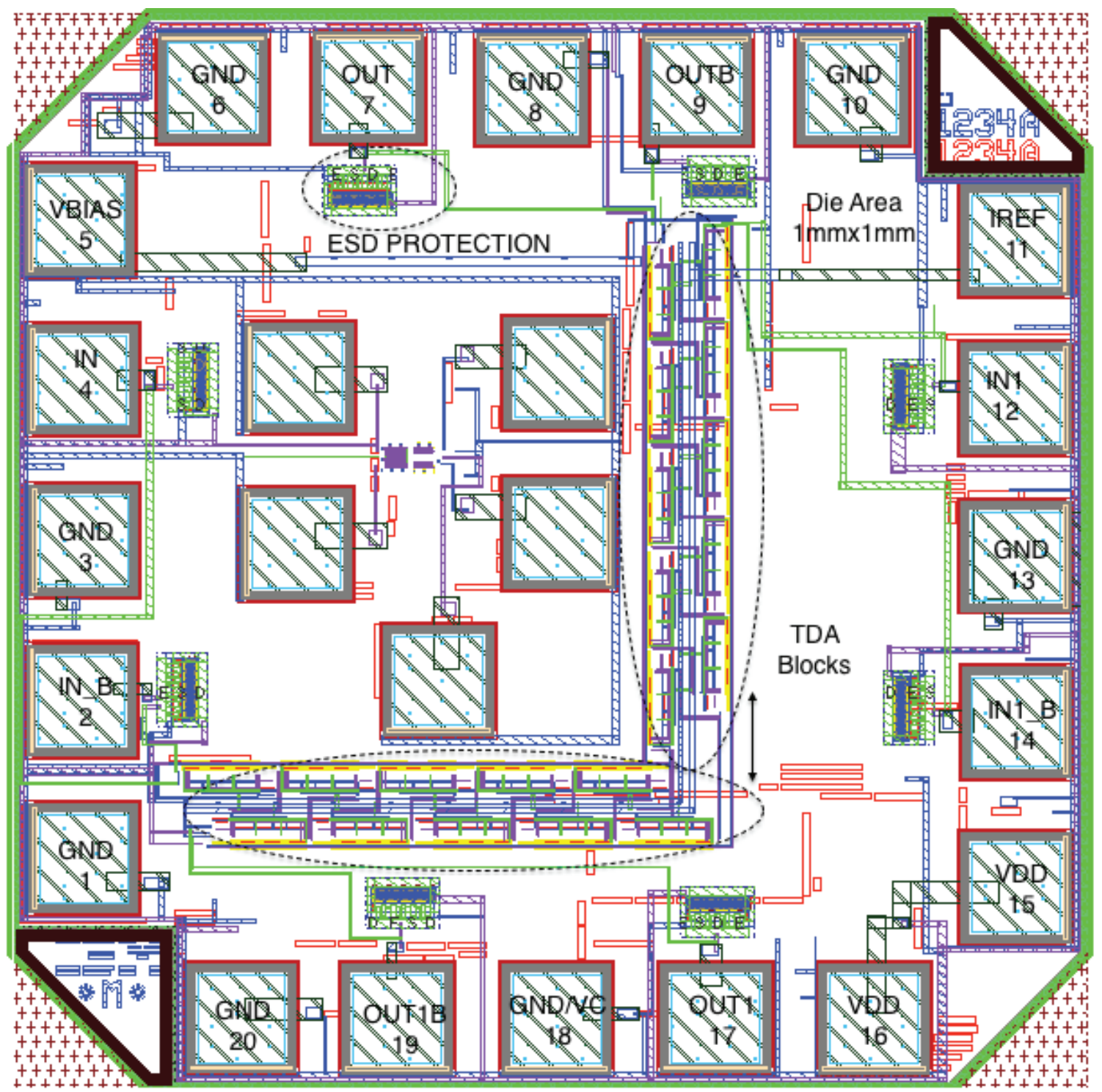

Figure 4.22: A $1 \mathrm{~mm} \times 1 \mathrm{~mm}$ die size is estimated and the layout is done. One can see the TDA chains that are highlighted. Internal ESD protection is also seen for signal inputs. There are twenty pins/bondpads present. 


\subsection{Simulation Results}

Simulation on the Time Difference amplifier was performed using the Spectre tool from Cadence and results are presented in this section.

The test bench for Cadence simulation is simple and is shown in the below figure 4.23 .

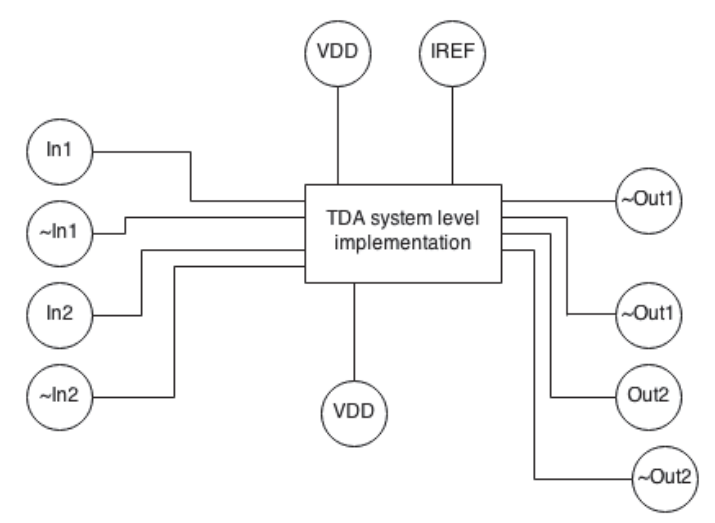

Figure 4.23: Simulation Test Bench

A transient analysis was run on the design. The period of the run being $2 \mu S$. The inputs to the device are step signals and the delay between two input signals are increased in steps of $10 \mathrm{pS}$ in subsequent runs. The gain that is offered was calculated using cross function in the cadence calculator. The two inputs are connected to a signal generator(Vpulse) which generates pulses of the required amplitude and frequency, the second signal generator also generates a similar pulse but is delayed with respect to the first. The output nodes that need to be plotted are selected and the delay output is calculated using the cross function. The cross function takes the threshold voltage and the cycle number on rising or falling edge as inputs and its output will be time. The delay out is calculated using,

$$
\text { Delay }_{\text {out }}=T_{\text {out } 1}-T_{\text {out2 }}
$$


Where $T_{\text {out1 }}$ and $T_{\text {out2 }}$ are computed using the cross function of the first cycle, with threshold voltage as 1 Volt for the two output nodes. Similarly the cross function is used for the input signal and equation is given as,

$$
\text { Delay }_{\operatorname{In}}=T_{\operatorname{In} 1}-T_{\operatorname{In} 2}
$$

The gain is computed as,

$$
\text { Gain }=\frac{\text { Delay }_{\mathrm{out}}}{\text { Delay }_{\mathrm{In}}}
$$

The below figure gives the waveform for an input delay of $100 p S$.

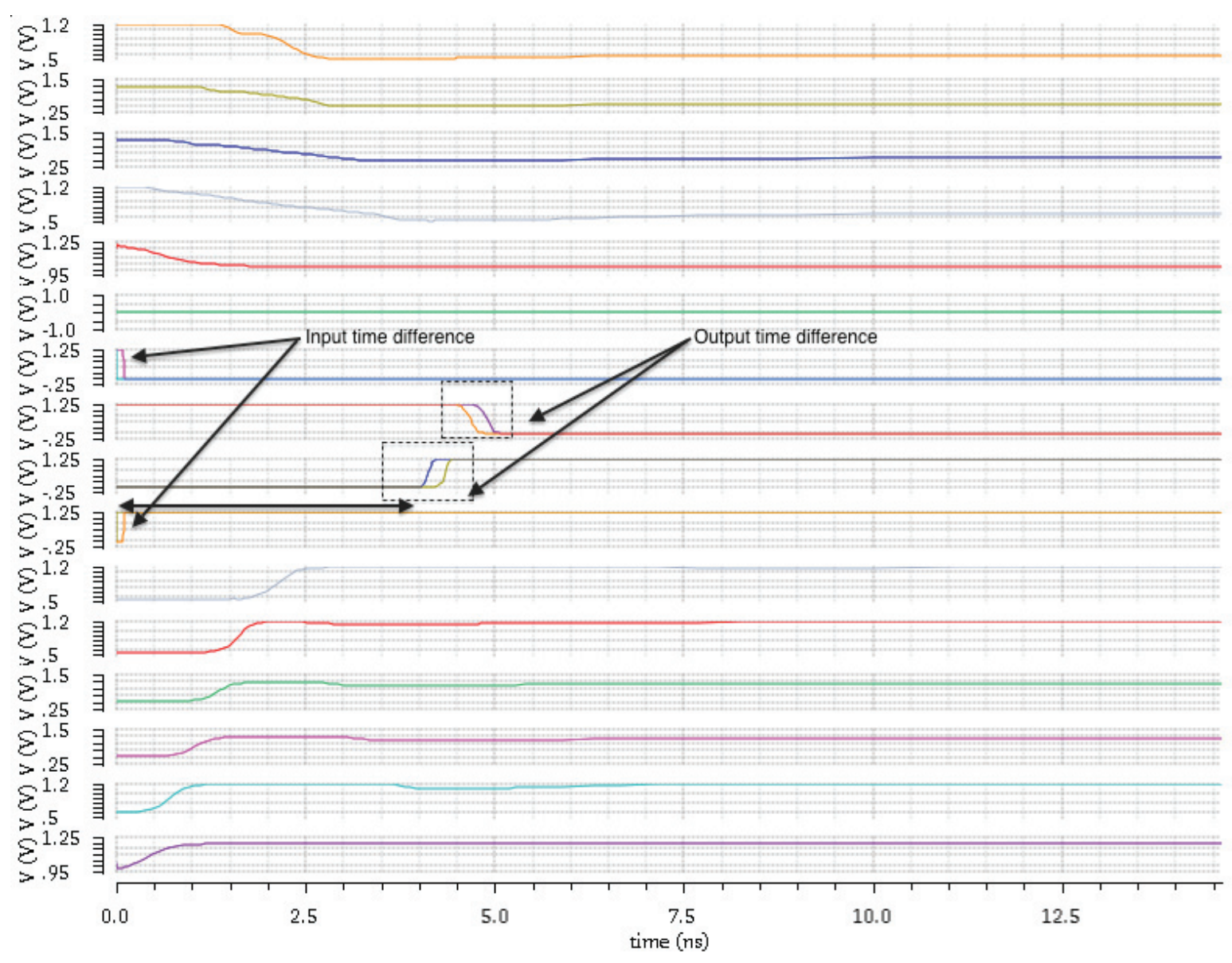

Figure 4.24: The output time difference is an amplified version of the input. The rest of the signals are the outputs of individual stages. 
As seen in the output waveform the input time difference is amplified by the TDA. The most important parameter for the TDA is its linearity. To estimate the linearity of the TDA a parametric simulation is performed. The time difference between the input signals are swept from $1 \mathrm{pS}$ to $2 \mathrm{nS}$ in steps of $20 \mathrm{pS}$ which amounts to about 100 points. The output time difference at each step is plotted. The resulting transfer function is shown in figure 4.26 It is seen that variations in gain are least when the input time delay is between $80 \mathrm{pS}$ to $1000 \mathrm{pS}$. The graph from figure 4.25 indicates the gain which is greater than 2 . 


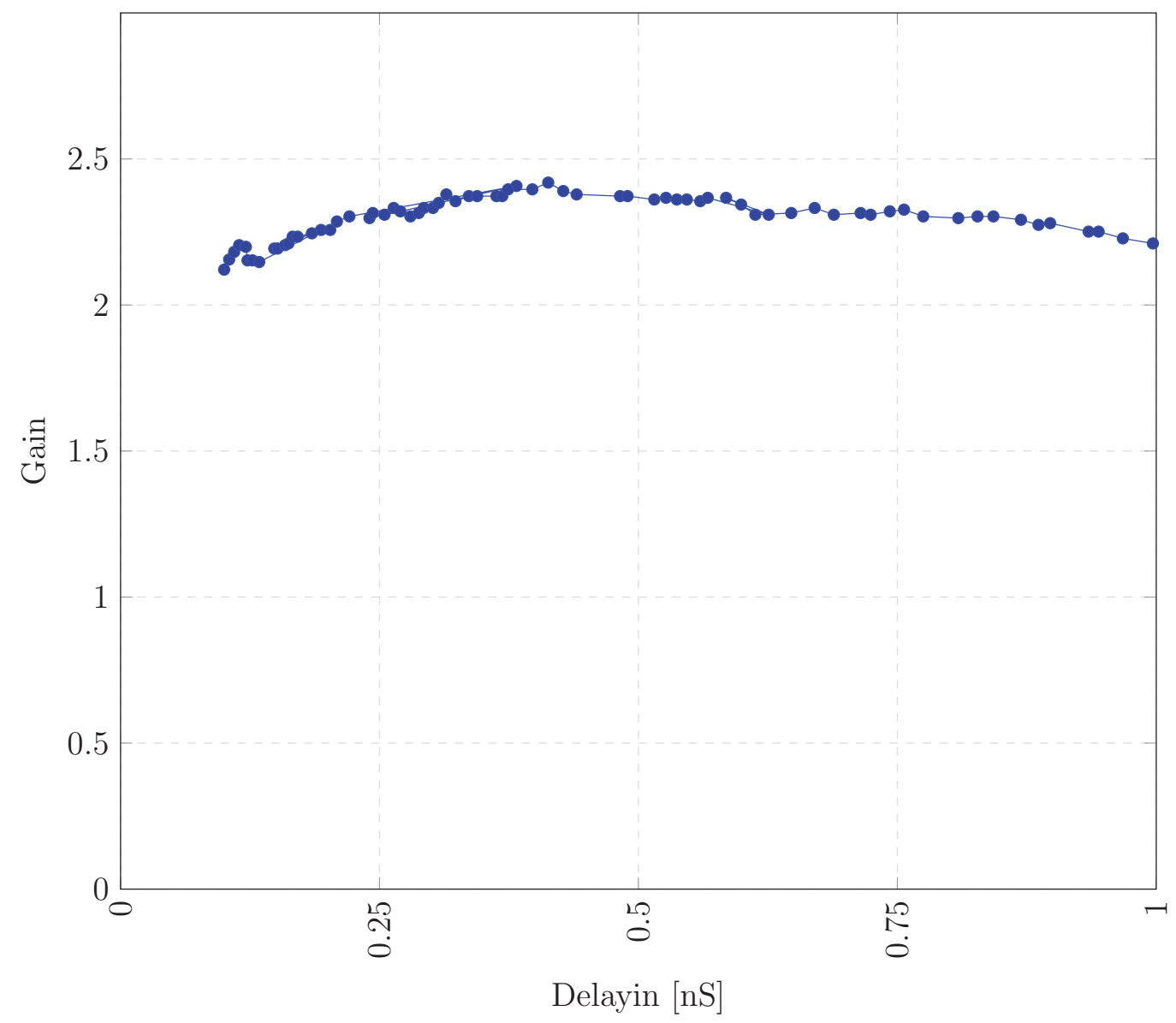

Figure 4.25: effective linear range is from $80 \mathrm{pS}$ to $1 \mathrm{nS}$, having a gain error less than $15 \%$ 


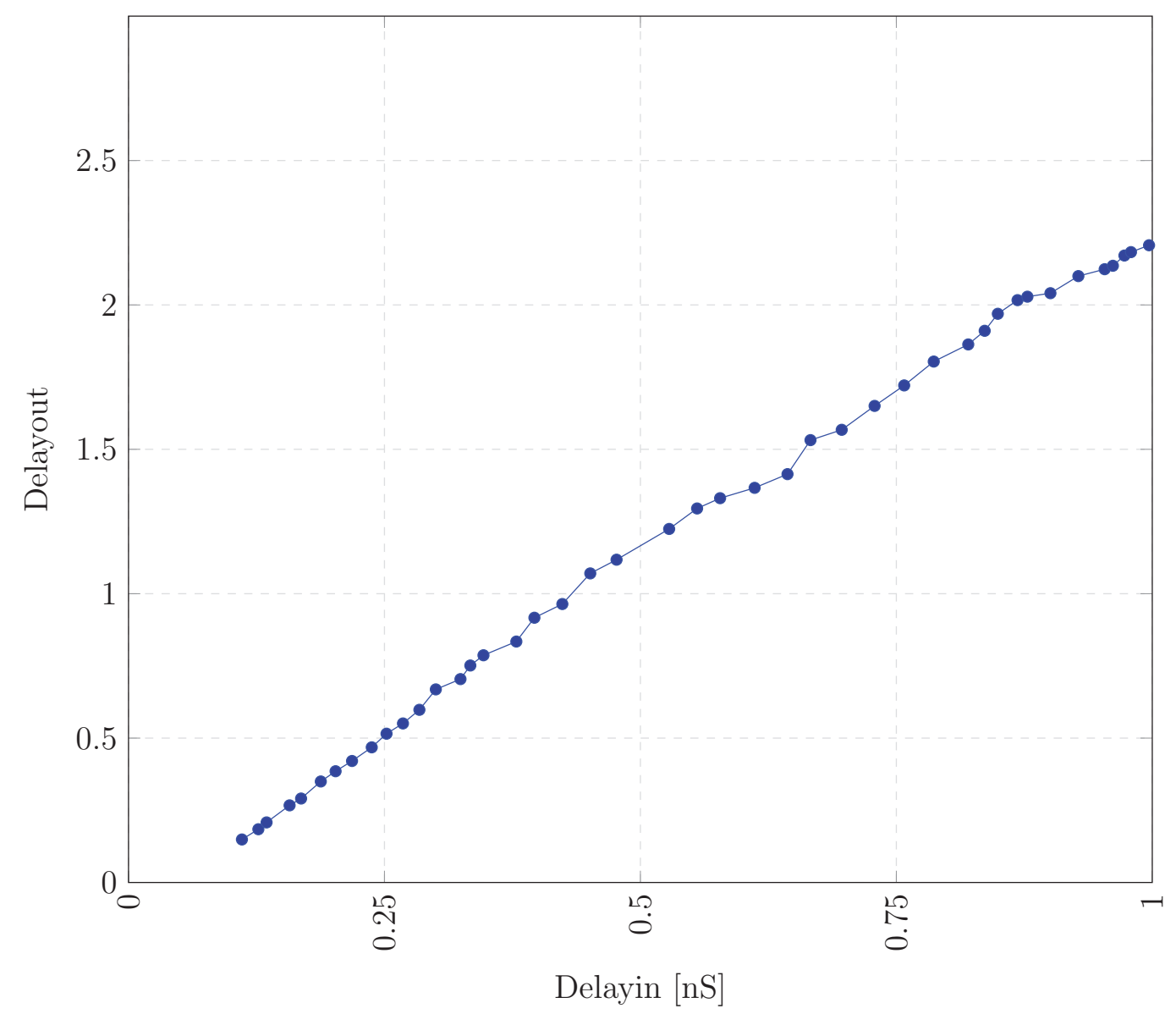

Figure 4.26: The output delay plot of the TDA is shown, linearly increasing, having a slope of 2 indicating the gain.

The next plot however shows the full sweep of the delay, where the delay is varied between $1 \mathrm{pS}$ to $2000 \mathrm{pS}$ in steps of $20 \mathrm{pS}$. 


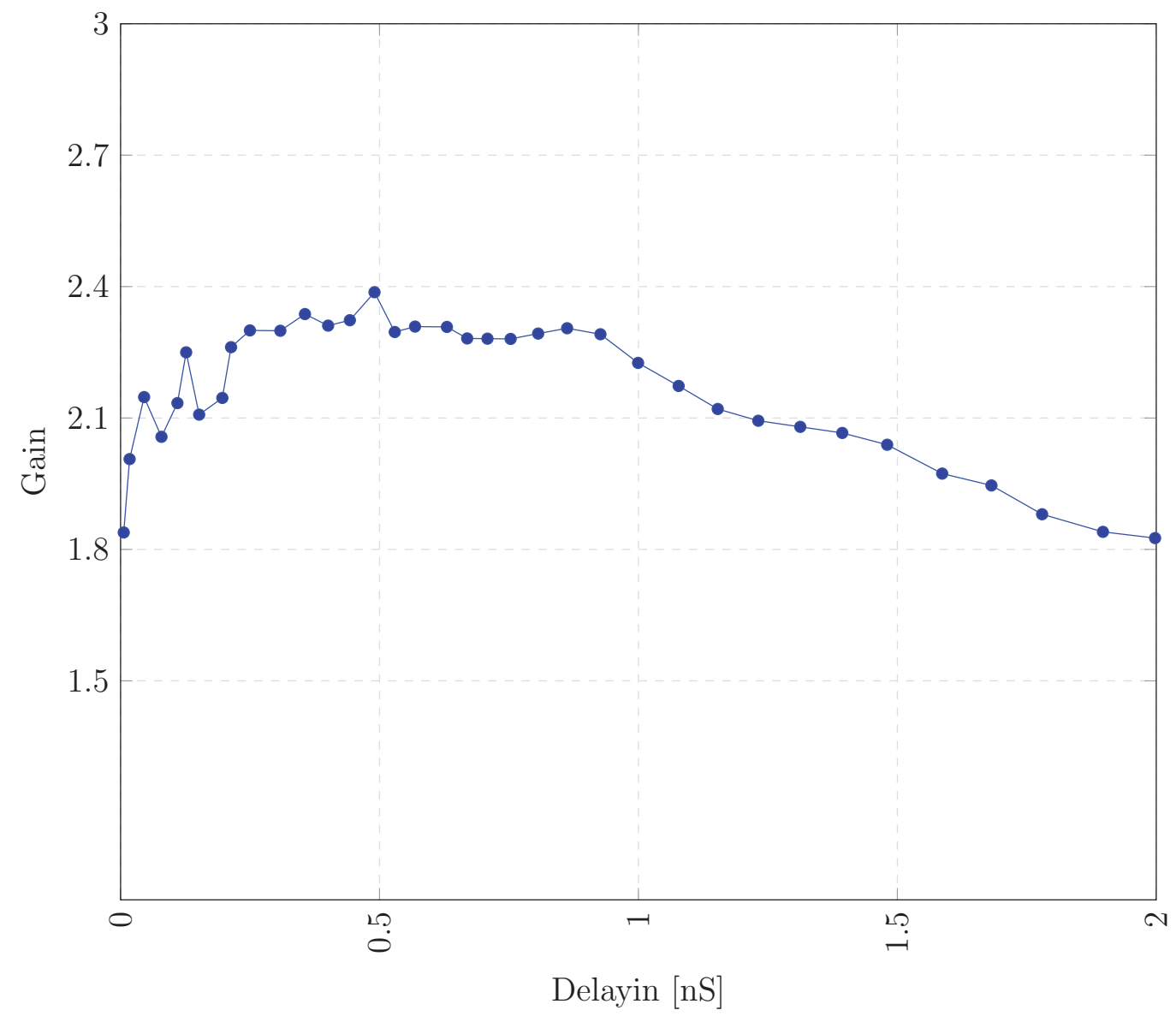

Figure 4.27: The input is varied from $10 \mathrm{pS}$ to $2 \mathrm{nS}$ and the transfer gain curve is observed.

Though the gain varies, it is about 2 on an average. As is the behaviour with any amplifier, it is seen that the with increase in the input time the amplifier the gain begins to reduce as the input delay is more than the switching time.

The output delay out can be modelled as a straight line whose equation is,

$$
y=m \cdot x+c
$$

where $m$ denotes the slope of the line here the gain of the amplifier. 


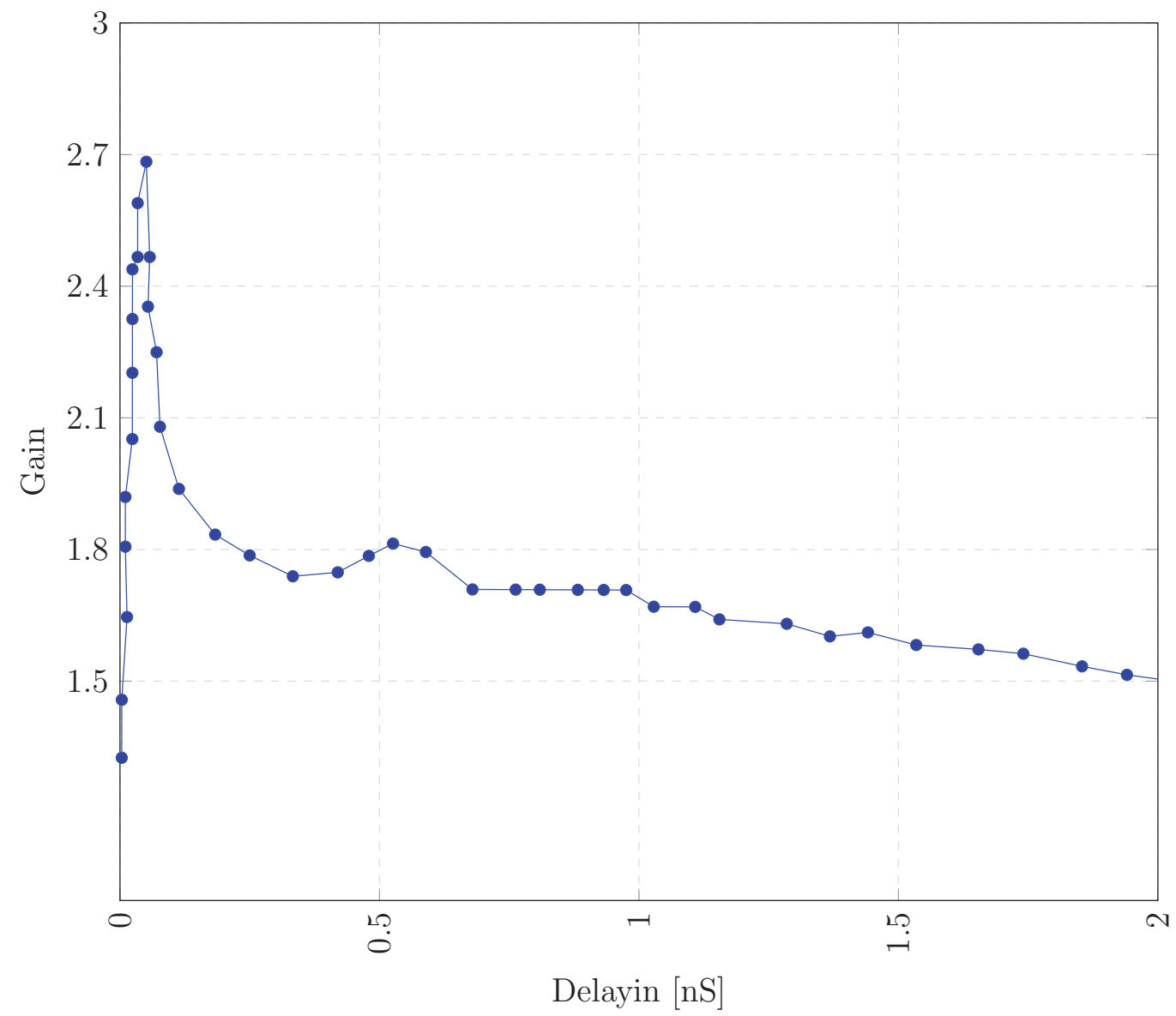

Figure 4.28: The input is varied from $10 \mathrm{pS}$ to $2 \mathrm{nS}$ and the transfer gain curve is observed at the fast corner at $-25^{\circ} \mathrm{C}$.

This design of the TDA is an open loop version which is one of its drawbacks. If the amplifier had feedback it there would be lesser variation in its gain. However this disadvantage can be overcome by using a DLL based feedback circuit that would sense the output time difference and sends this time information to a charge pump. The charge pump would convert the time difference to voltage and change the bias, resulting in minimizing the gain errors. 
The delay cell was characterized and longest delay were 1.3ns and shorter delay is $83 \mathrm{pS}$. The simulation result is shown in Figures 4.29 and 4.30 respectively.

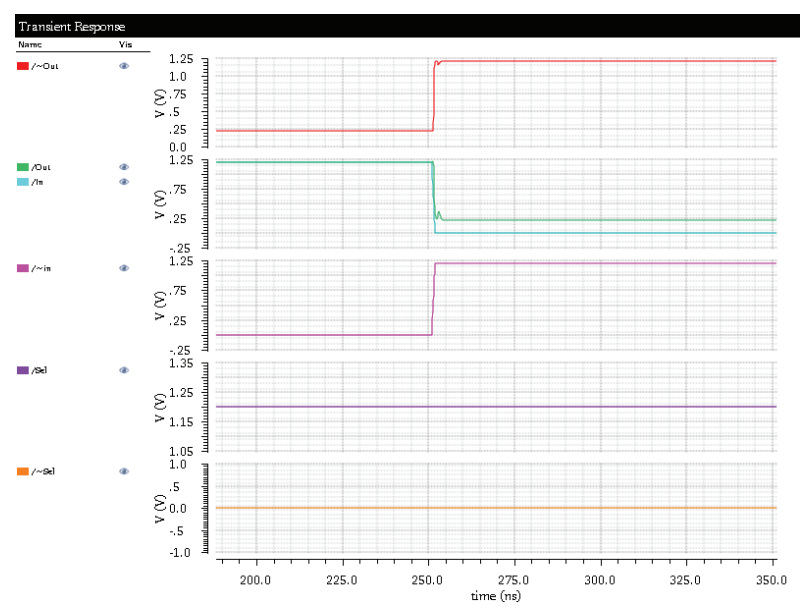

Figure 4.29: Short Delay characterization

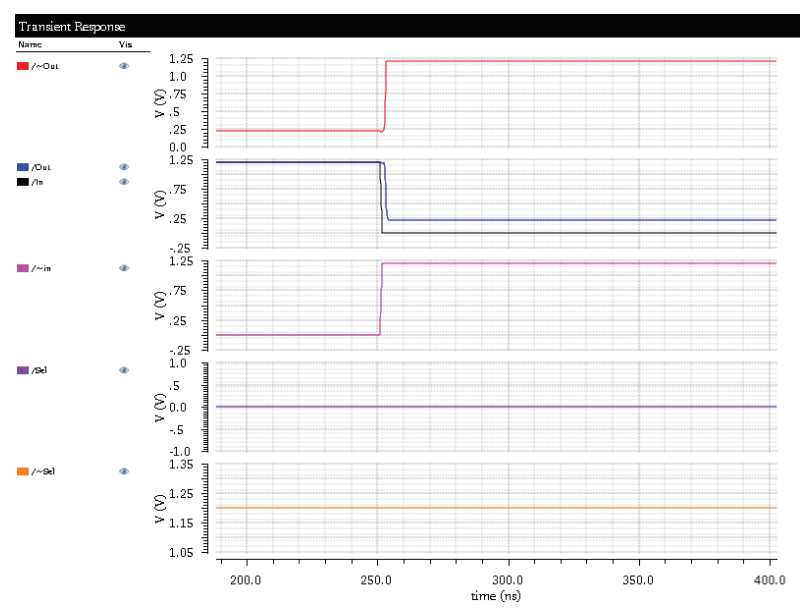

Figure 4.30: Long Delay characterization

The variations in the gain is due to the switching time of the multiplexer in the device. Upon characterizing the multiplexer in this design, the switching time was found to be $385 \mathrm{pS}$. If this switching time can be decreased or if the circuits switch faster, then the variations in the gain or the step type of degradation in gain can be minimized. 


\section{Chapter 5}

\section{Testing methodology and Design}

\subsection{Test Board Circuit Design}

To be able to test the device a Printed circuit board was fabricated with minimal test circuit so that the required inputs and calibration circuits are available to test the device. The block diagram of the support circuit for testing the TDA device and its connection to the device are shown in figure 5.1 The support circuit has two programmable delay devices SY100EP196VTG that can be programmed with 9 bit digital inputs to provide pSec delays in discrete steps of $15 \mathrm{pS}$. The devices also have an analog input controller by a multi turn trimpot with which the selected delay can be fine tuned. The two delay devices accept differential inputs and can be connected to two different sources from a function generator. These two inputs can also be bridged to a single input so that the delay devices have the same input signal and this would be most common test setup. The delay device has an ECL output stage and therefore is followed by a LVECL to LVCMOS converter that provides an output suitable to the DUT. 


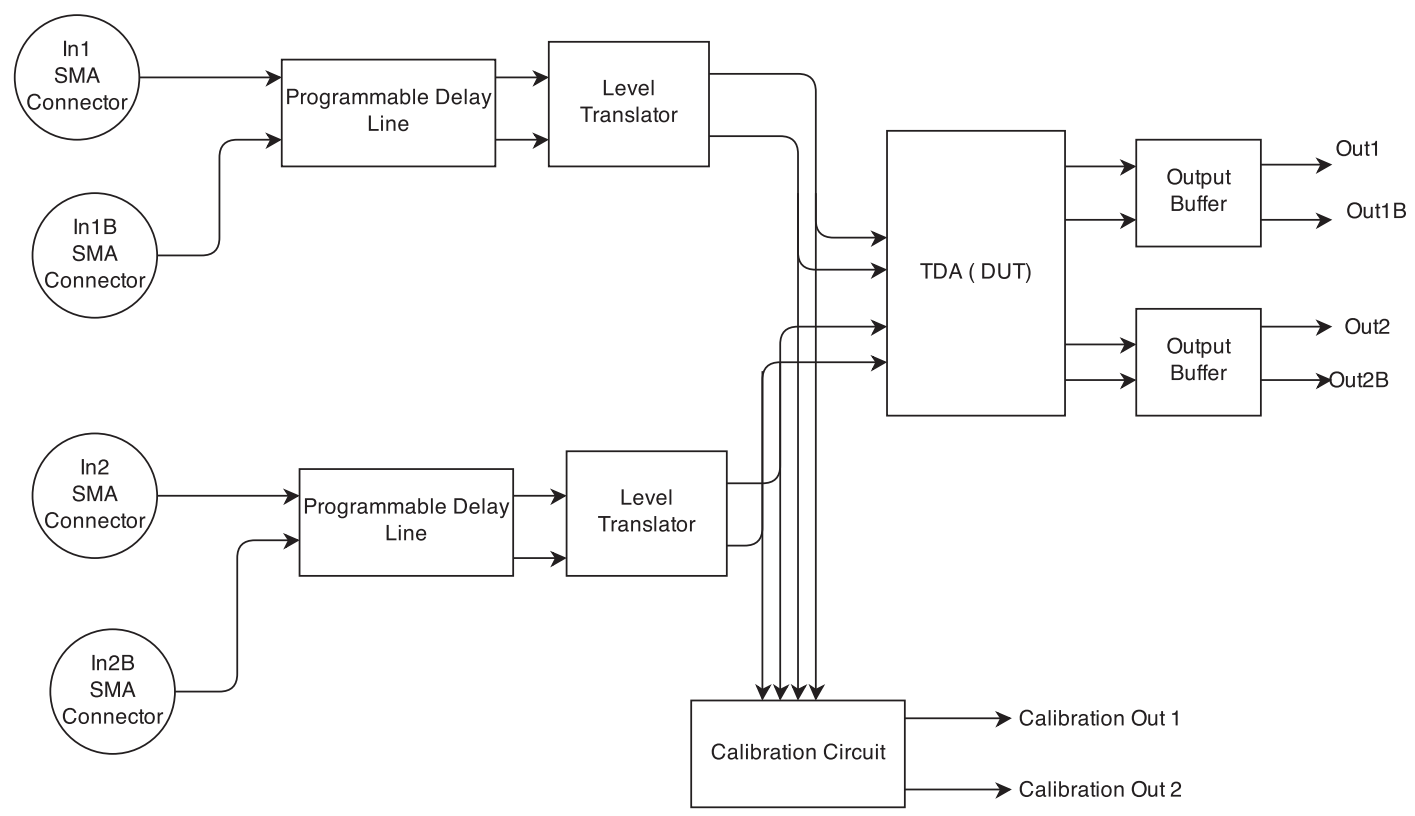

Figure 5.1: $50 \Omega$ matched SMA connectors are used for feeding the input signal, signals are fed to a delay chip. To match the output levels they are level translated and fed to the the DUT. A calibration circuit is used to match the delay.

This stage removes the DC offset that is present in the ECL output and the resulting waveform is compatible to the input stage of the DUT.

The digital inputs to the delay device is selected by jumpers on the board. Even when the delay chosen is the same for both devices the resulting output will have minor differences and therefore a calibration circuit is included that helps compare their outputs and zero them out.

The output delay of the chip in pico second [11] is given by,

$$
\text { Delay }=2200+(10 \times \operatorname{value}(D[9: 0])+\operatorname{delay}(\text { Ftune }) .
$$

A simple pair of high speed XOR gates act as a comparator between the two input signals that is helpful in calibrating the test circuit. The XOR gate is the calibration circuit depicted in the figure 5.1. The property of an XOR gate to 
provide a true output when the two inputs are different makes it useful as a phase detector that detects a change in phase or the delay between the two signals shown in table 5.1.

\begin{tabular}{|c|c||c|}
\hline $\mathrm{A}$ & $\mathrm{B}$ & $\mathrm{Y}$ \\
\hline 0 & 0 & 0 \\
\hline 0 & 1 & 1 \\
\hline 1 & 0 & 1 \\
\hline 1 & 1 & 0 \\
\hline
\end{tabular}

Table 5.1: XOR Gate truth table

To calibrate the test support circuit the delay from both devices are set the same and the same input is provided to both devices. The analog signal input to one or both devices are adjusted with the trimpots provided until the comparator circuit output is zero. The power supplies, bias and current references were not included on the test board and these are from external devices that provide better working conditions to the DUT compares to on board regulators and current sources. However addition of these power regulators, current sources and voltage reference would made the board a standalone device that can work off any standard power supply. All input and output signals are terminated onto SMA connectors on the board to ensure proper connectivity to the signal generator and the measuring device. 


\subsection{Printed Circuit Board Design}

This section describes the Printed Circuit Board design that is used for system integration for testing the TDA chip. The tool here used is the Cadence design suite for printed circuit boards. OrCad is used for designing schematics and the Allegro design tool is used for the layout. The PCB is manufactued at MyRo PCB Ottawa.

\subsubsection{PCB Flow}

The schematics were captured the OrCad tool. Required symbols for custom devices used were generated and added to libraries, as they were not already part of the standard Orcad component libraries. The appropriate package was chosen for each component in the design. The footprint library contains PCB footprints to different component packages. Footprints were created for custom packages like the TDA DUT. A netlist was generated from the schematic after running a Electrical Rule Check on the design. The design was then imported into the allegro tool. The board stackup and the dimensions were fixed. Components were placed according to the constraints. Post such placement the nets were routed manually. A design rule check was run to rule out violations of specification laid out by the PCB manufacturer. When all DRC errors were cleared, a netlist was generated from the routed board and compared for no deviations against the one generated from the schematic earlier. Gerber files were generated from the routed board were sent to the manufacturer for fabrication [49]. 


\subsubsection{PCB Specifications}

\begin{tabular}{|c|c|}
\hline Dimension & $83 \mathrm{~mm} \times 70 \mathrm{~mm}$ \\
\hline Layers & 4 \\
\hline Signal layers & 2 (Top, Bottom) \\
\hline Power Planes & $2($ PWR, GND) \\
\hline Copper & $1 \mathrm{Oz}$ \\
\hline Finishing & Immersion Gold \\
\hline RoHS & Complaint \\
\hline Component placement & Top layer only \\
\hline
\end{tabular}

Table 5.2: PCB Specifications

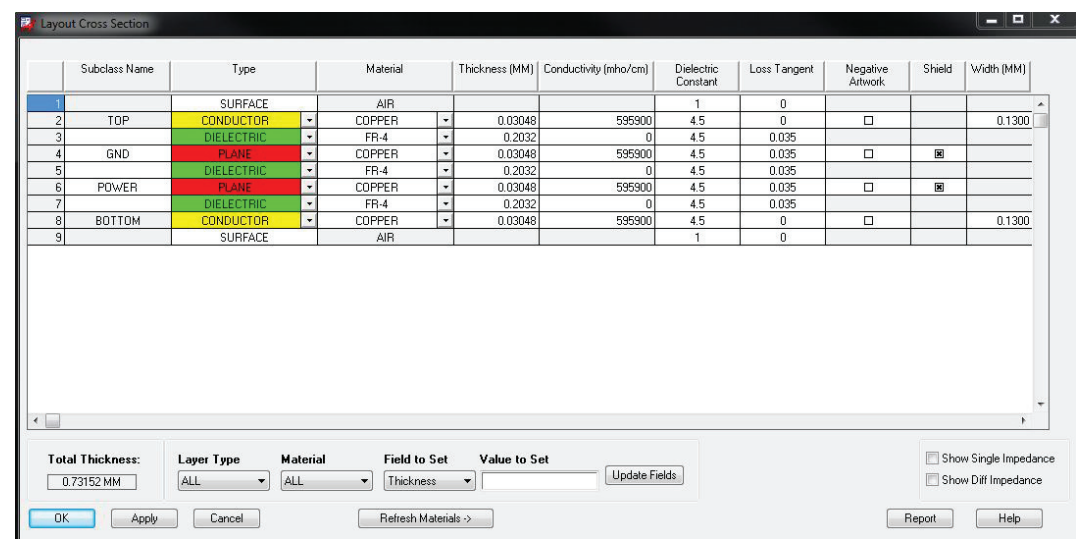

Figure 5.2: Board Stack Up

Only the TOP and BOTTOM layers were used for signal routing, power planes were in the inner layers. No blind or buried vias were used in this design. All components were placed at the top layer and lower layer was kept free to enable easier mounting on the wirebonding machine. The TDA device is leadless and hence needed wirebonding. 


\subsection{PCB design details}

Care was taken during placement and routing so as to minimize cross talk, delays which can introduce measurement errors. Trace lengths were kept minimum. Trace widths were chosen based on the type of signal carried. Via optimization was performed. Traces used for differential pairs were matched. Serpentine routing $[50,51]$ was done to length match these lines. The longest trace length is calculated and serpentine routing is done to the the shorter line to achieve a length match. To reduce noise and provide good return paths to ground, a copper pour was done covering all the areas where there were no traces. The pour is connected to the ground plane. PCB floor plan, pre routing and post routed images of the PCB are shown in Figure 5.3 and 5.4. Refer appendix C for gerber data.

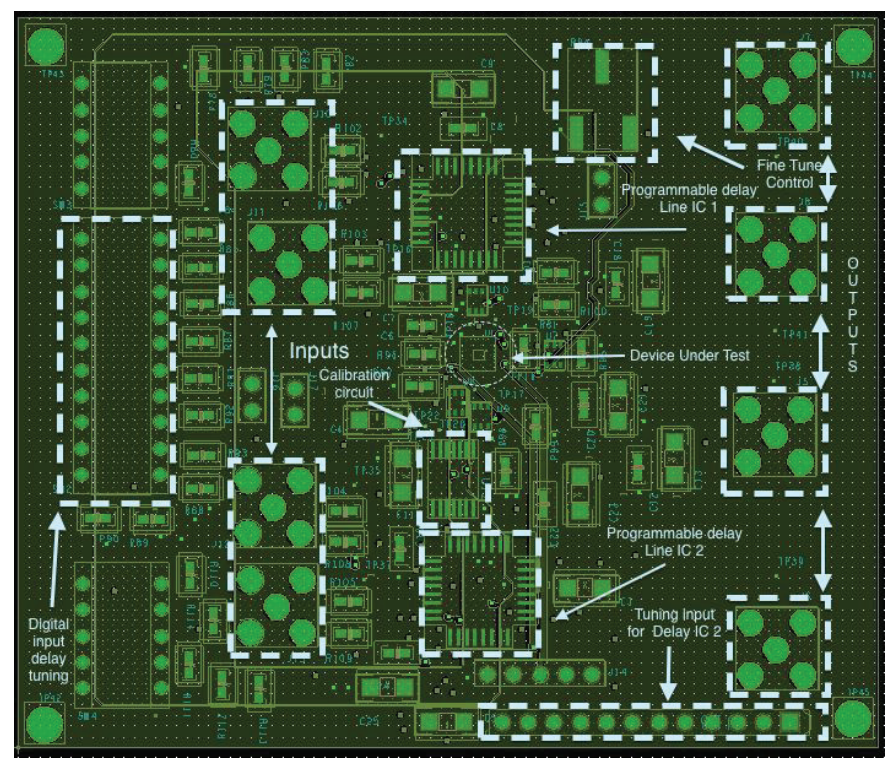

Figure 5.3: PCB Layout without routing 




Figure 5.4: PCB Routing 


\subsection{Printed Circuit Board Bring up Plan}

Board revision : V 1.0 This section describes the board bring up plan for testing the printed circuit board after assembly.

\subsubsection{ESD Caution}

All devices are ESD sensitive. It was ensured that the all assembly, test equipment and work area were ESD safe. Care was taken while handling the device/board to prevent ESD failures. The first stage was to validate the support circuitry. In this step all device except the TDA device under test were populated. Signals as per test procedure were injected and the proper working of the support circuitry was verified.

\subsubsection{Equipments required from board bring up}

- Fluke Digital Multimeter

- Agilent Infinium 54855A 6 GHz Oscilloscope

- Keithley 2602 /2636 Dual Power supply

- Agilent 81150A Pulse Function Arbitrary Generator.

- Keithley 6220 Precision Current Source. 
The table below describes the tests that are done at the time of board bring

up.

\begin{tabular}{|c||c||}
\hline Test flow steps & Test Name \\
\hline 1 & Cold Resistance test \\
\hline 2 & Power Supply Test \\
\hline 3 & Power Sequencing Test \\
\hline 4 & Delay Line calibration \\
\hline 5 & Silicon Mounting and Bring up \\
\hline 6 & Board bring up complete \\
\hline
\end{tabular}

Table 5.3: Test flow

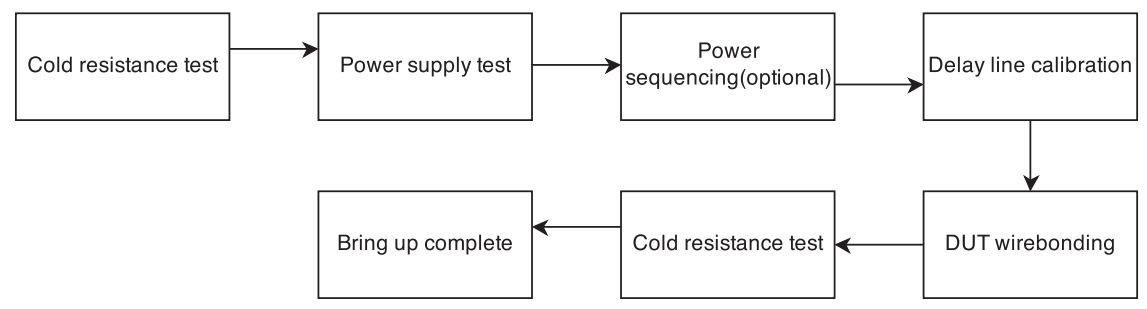

Figure 5.5: The block diagram shows a procedure to bring up boards. The various steps are mentioned 


\subsubsection{Cold Resistance Test}

This test is done to rule out shorts on the board after assembly. The resistances between the GND and all supply are checked prior to powering the board.

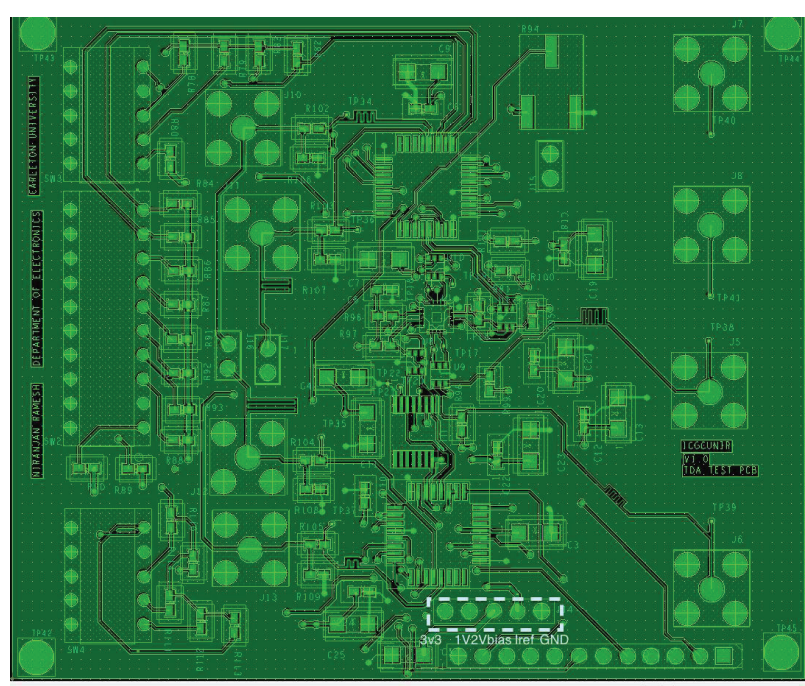

Figure 5.6: PCB Power Rails

\begin{tabular}{|c||c|c|}
\hline Pin Number & Rail & Resistance in $\Omega$ \\
\hline J14.1 & $3.3 \mathrm{~V}$ & $100 \mathrm{k}$ \\
\hline J14.2 & $1.2 \mathrm{~V}$ & $2.4 \mathrm{M}$ \\
\hline J14.3 & Vbias & $2.4 \mathrm{M}$ \\
\hline J14.4 & Iref & $4.5 \mathrm{k}$ \\
\hline
\end{tabular}

Table 5.4: Cold Resistance Test Result 


\subsubsection{Power Supply Test}

This test is performed to measure that the power supply rails are not loaded by the circuitry and supply currents remain within limits. Figure 5.8 gives the test points to be probed.

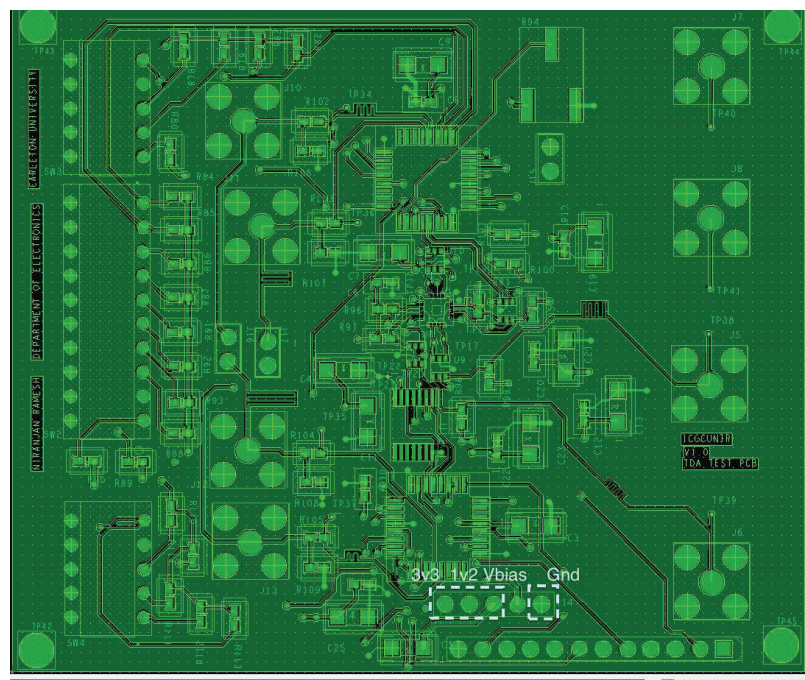

Figure 5.7: PCB Power Rails Test

\begin{tabular}{|c||c|c|}
\hline Pin Number & Rail & Voltage in $V$ \\
\hline J14.1 & $3.3 \mathrm{~V}$ & 3.28 \\
\hline J14.2 & $1.2 \mathrm{~V}$ & 1.18 \\
\hline J14.3 & Vbias & 0.7 \\
\hline
\end{tabular}

Table 5.5: Voltage Rail Test Results

The following were setting used for the power supply in order to ensure safe operation. 
1. Power supply set to $3.3 \mathrm{~V}$ and $1.2 \mathrm{~V}$ with current limit of $20 \mathrm{~mA}$.

2. Vbias set to $0.7 \mathrm{~V}$ with current limit of $2 \mathrm{~mA}$

3. Iref is set to $1.2 \mathrm{~mA}$ with voltage limit of $1.2 \mathrm{~V}$.

After power on the supply rails were checked by using a multimeter and all values were verified to be within working limits.

\subsubsection{Power Sequencing}

When multiple supply rails are involved it is common practice to do a power sequencing test, which measures the sequence and the time taken for each voltage rail to reach its final value during power on/ poweroff. However for this application the power sequencing is not mandatory. 


\subsubsection{Delay line IC Calibration}

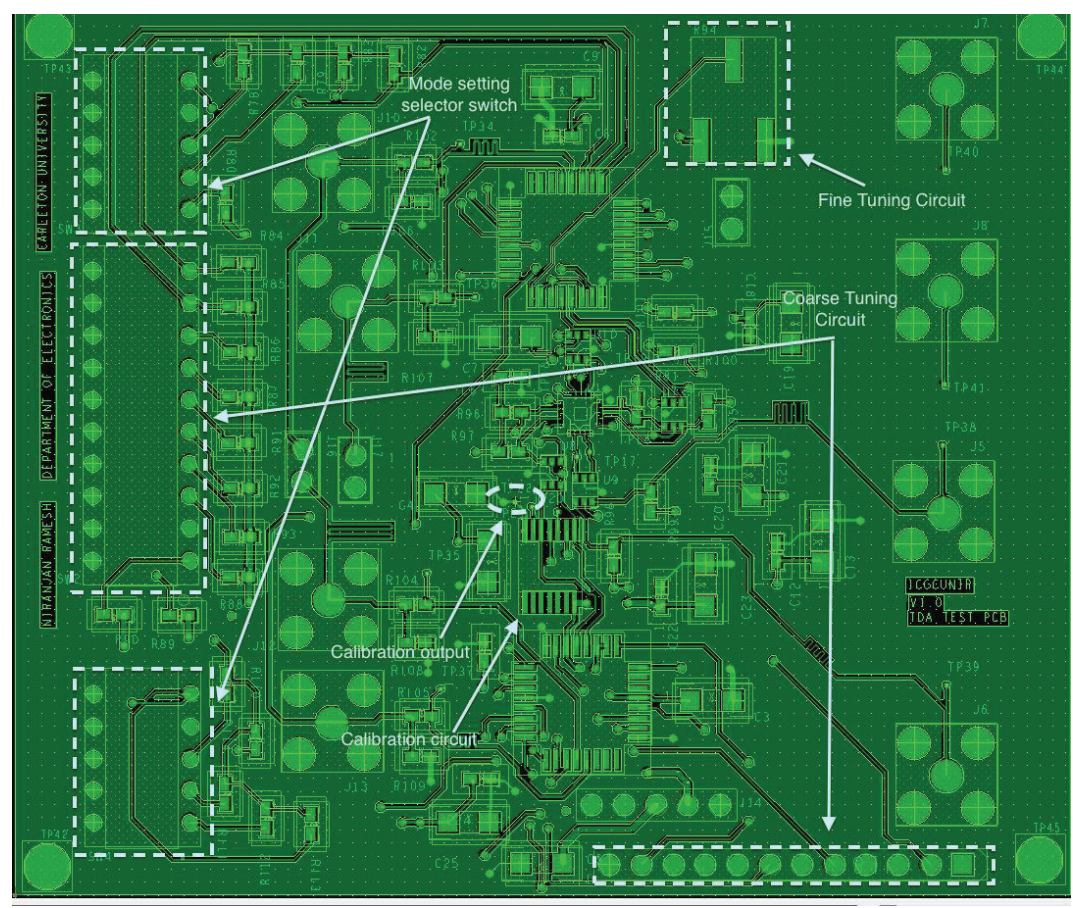

Figure 5.8: PCB Power Rails Test

The delay line IC chosen is from Micrel, two ICs were used to minimize any offset errors in the input to the DUT. The output signals from these two devices need to be calibrated.

The mode selector switch enables to set the chips individually to either be used as a fixed delay or a programmable delay. The first chip is made as a programmable delay element and the second one as a fixed delay. Both chips have a delay offset of $2000 \mathrm{pS}$. The circuit is calibrated by selecting a fixed delay for both devices and when a signal is injected the output of the calibration circuit is verified to be a zero. If not the delays are fine tuned until the delays are matched. This is an important part of the board bring up plan to minimize measurement errors due to input offsets. 


\subsubsection{Silicon Bring Up}

The DUT was not packaged to any standard integrated circuit plastic package, instead the bare die was wirebonded directly to the PCB. Extreme caution was used during handling of the device as it is vulnerable to ESD damage. A cold resistance test was performed in order to ensure that the DUT had not inherent shorts between its power rails. Input and output signals were also verified for no shorts between each other and supply rails. The device was then powered on and the voltage rails were verified to be within tolerable limits and that the current drawn was close to expected values.

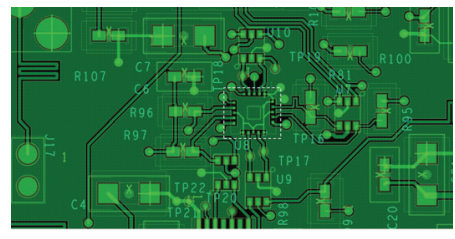

Figure 5.9: DUT

\begin{tabular}{|c||c|c|}
\hline Pin Number & Rail & Resistance in $\Omega$ \\
\hline J14.1 & $3.3 \mathrm{~V}$ & $100 \mathrm{k}$ \\
\hline J14.2 & $1.2 \mathrm{~V}$ & $2.4 \mathrm{M}$ \\
\hline J14.3 & Vbias & $2.4 \mathrm{M}$ \\
\hline J14.4 & Iref & $4.5 \mathrm{k}$ \\
\hline
\end{tabular}

Table 5.6: Cold Resistance Test Result after DUT Populated

This concludes the board bring up procedure. 


\subsection{Time Difference Amplifier Linearity Test}

In this section the TDA is tested for linearity. The test setup involves the following equipments

- Agilent 81150A Pulse Function Arbitrary Generator.

- Agilent Infinium 54855A 6 GHz Oscilloscope

- Keithley 2602 /2636 Dual Power supply

- Keithley 6220 precision current source.

The test procedure involves applying input signals with the following specifications.

1. Square pulse with amplitude of $1.2 \mathrm{~V}$ or $3.3 \mathrm{~V}$.

2. Rise time of $2.5 n S$ or lesser

3. Frequency of the pulse can be $1 \mathrm{Mhz}$ or lesser, as the frequency of the signal is not important for this application.

The delay between the two signals are selected using the 9 bit select signals of the programmable channel. The signals are measured at the output SMA connectors, with a 4 channel oscilloscope. The delay between the two input signals and the delay between the two output signals are measured and the gain is calculated. The input delay is swept in steps of $15 \mathrm{pS}$. For fine delay tuning the trimpot is used and for coarse delays, the jumper settings are changed. Input and output delays and calculated gains are tabulated and graph is plotted - input time difference versus the output time difference. The linearity and gain can be seen from this graph for the entire input range. 


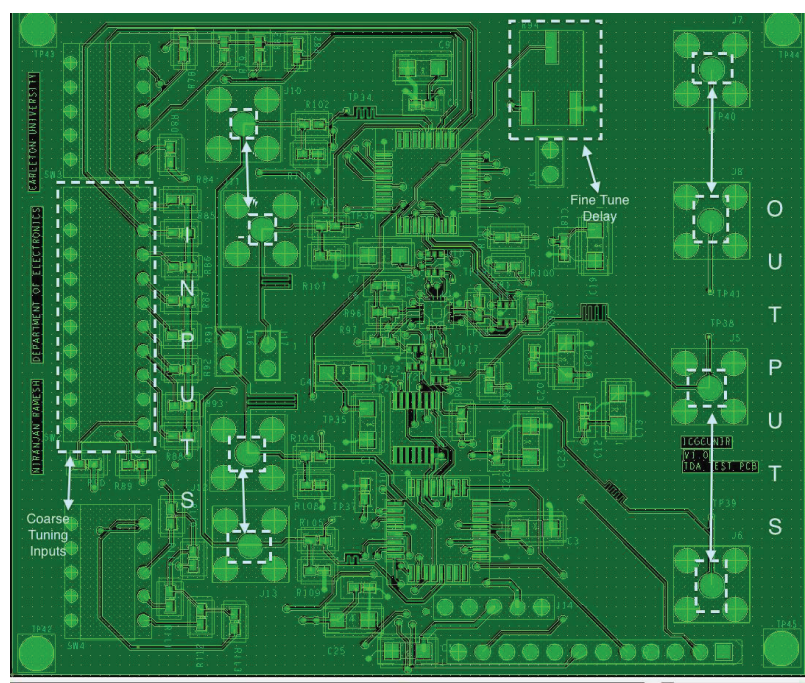

Figure 5.10: Linearity test

Figure 5.10 shows the test points for measuring the inputs and the outputs. 


\section{Chapter 6}

\section{TDA Testing and Debug}

This chapter describes detailed test setup and failure analysis of the DUT. The device failed to work after a brief amout of time and did not match simulation

results, due to ESD diodes that were breaking down clamping the output or inputs to either power rails. Various debug methods are employed and are discussed here. The test setup is first validated and verified on the PCB that is designed without the DUT populated. Once the test bench is verified the DUT was populated and the tests were carried out.The DUT is wirebonded at Carleton University to the board, and the testing is done.

A precision multiple output power supply, feeds the device with the two DC voltages of $1.2 \mathrm{~V}$ and $3.3 \mathrm{~V}$. The Vref signal is also derived from the same device and is set to $0,8 \mathrm{~V}$. The Precision current source is set to $1.2 \mathrm{~mA}$ at a compliance voltage of $1.2 \mathrm{~V}$ to provide the $\mathrm{DC}$ bias. A single channel pulse generator is connected to input of the test board and the jumper that makes the input common to both devices is populated. The support circuit is first calibrated as described above. The input source is selected to be a step signal with rise time of $1 \mathrm{nS}$. The output 


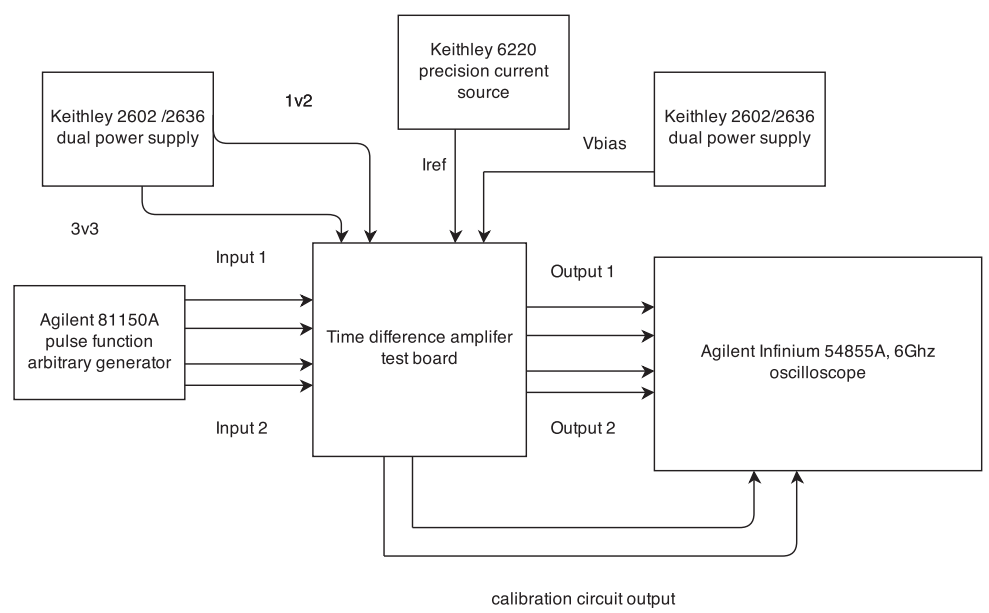

Figure 6.1: Test bench block diagram revealing all the measurement equipments required

voltage of the signal generator is selected to match the requirements of the delay device.

The delay between the input signals are varied during the test by varying the digital input of one of the delay devices using the jumpers for that channel. For every value of the input delay, output delay is measured using a 6Ghz Oscilloscope and recorded. 


\subsection{Test Bench Setup}

This section describes the test setup that is used to test the DUT.

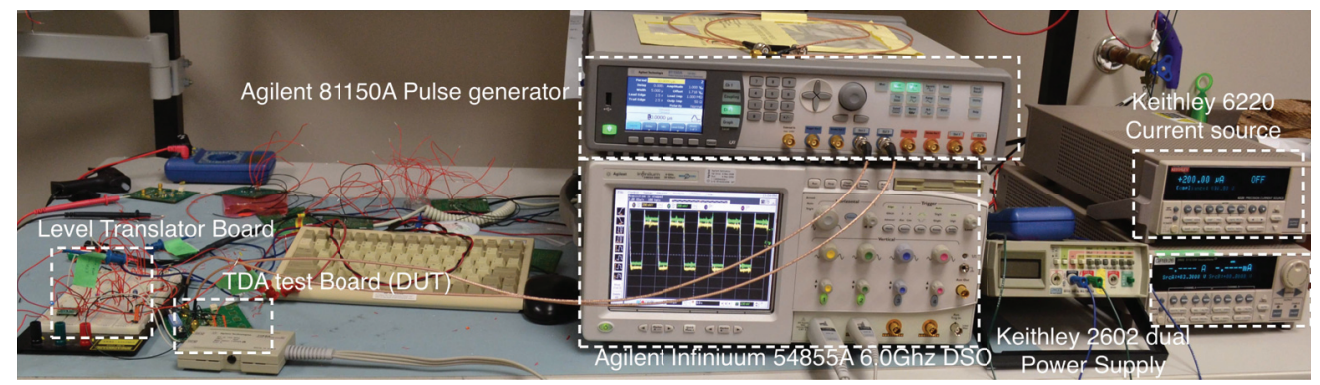

Figure 6.2: Test bench setup showing the precautions taken for ESD by providing a mat The required compatible instruments for this experiment is shown.

The test bench setup is shown in figure 6.2.

The DUT is tested by applying an input signal whose time difference can be tuned. The differential input signals are fed from the the Aglient 81150A pulse generator, the time differences are tunable in the TDA test board. The signals from the test board are in LVPECL levels and hence level translated to LVCMOS levels and fed back to the TDA to the input of the DUT. The input delay is tuned in steps of $10 \mathrm{pS}$ and is also fine tuned to get delays as small as $5 \mathrm{pS}$.

The test board is powered with the keithley 2602 dual power supply powering up the board with $3.3 \mathrm{~V}, 1.2 \mathrm{~V}$ and $700 \mathrm{mV}$ is obtained by another Keithley supply.

The bias current is precisely set by the Keithley 6220 Precision current source that supplies $200 \mu \mathrm{A}$, voltage limited to $800 \mathrm{mV}$. 


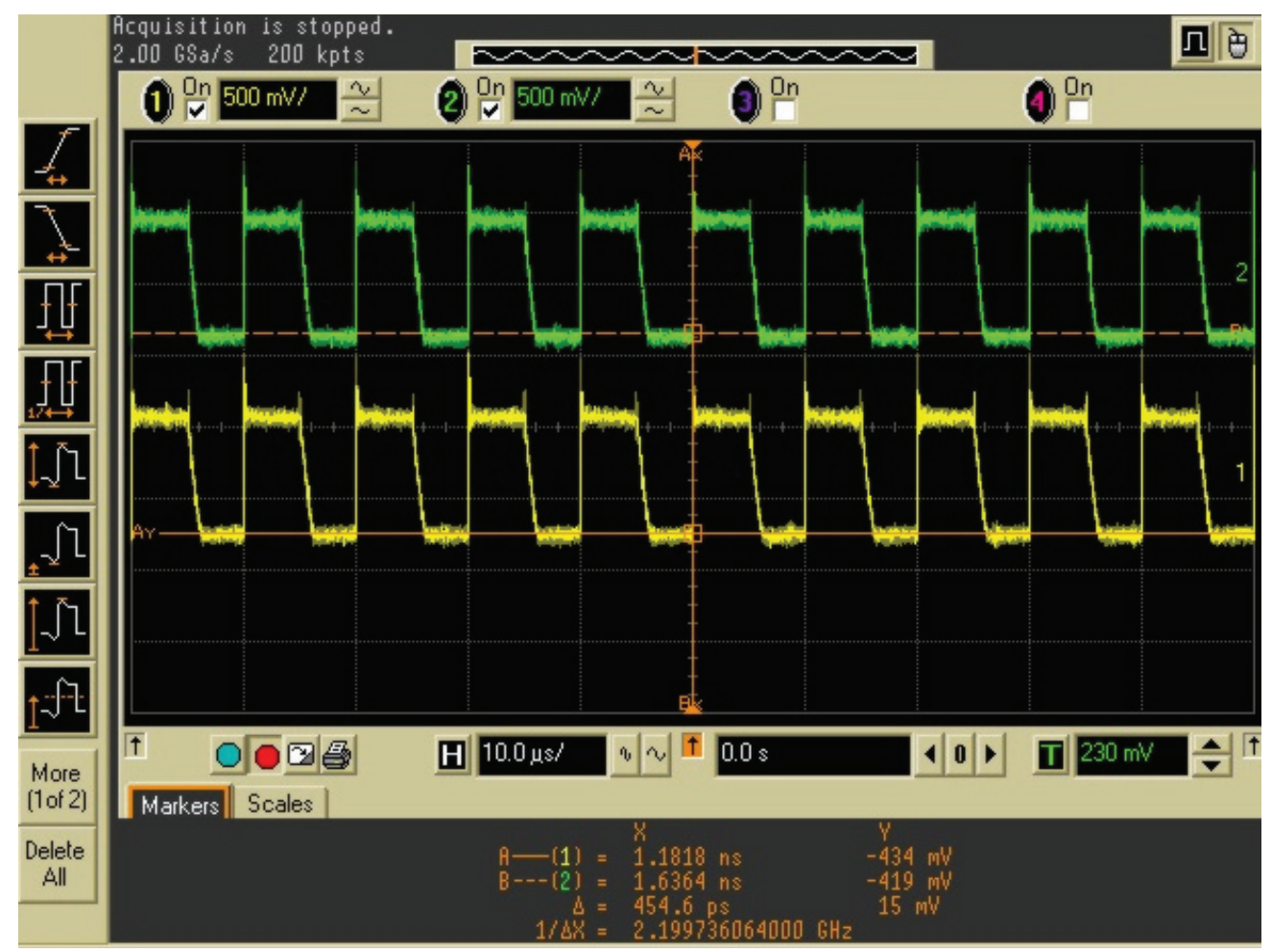

Figure 6.3: Input Waveform Applied to DUT

The waveform shown in Figure 6.3 is applied to the DUT. Markers are enabled to see the input time differene to be close to $454 \mathrm{pS}$, that is tuned by the TDA test board. These levels are are LVCMOS.

A zoomed in version of the waveform is shown in figure 6.4 for clarity. 


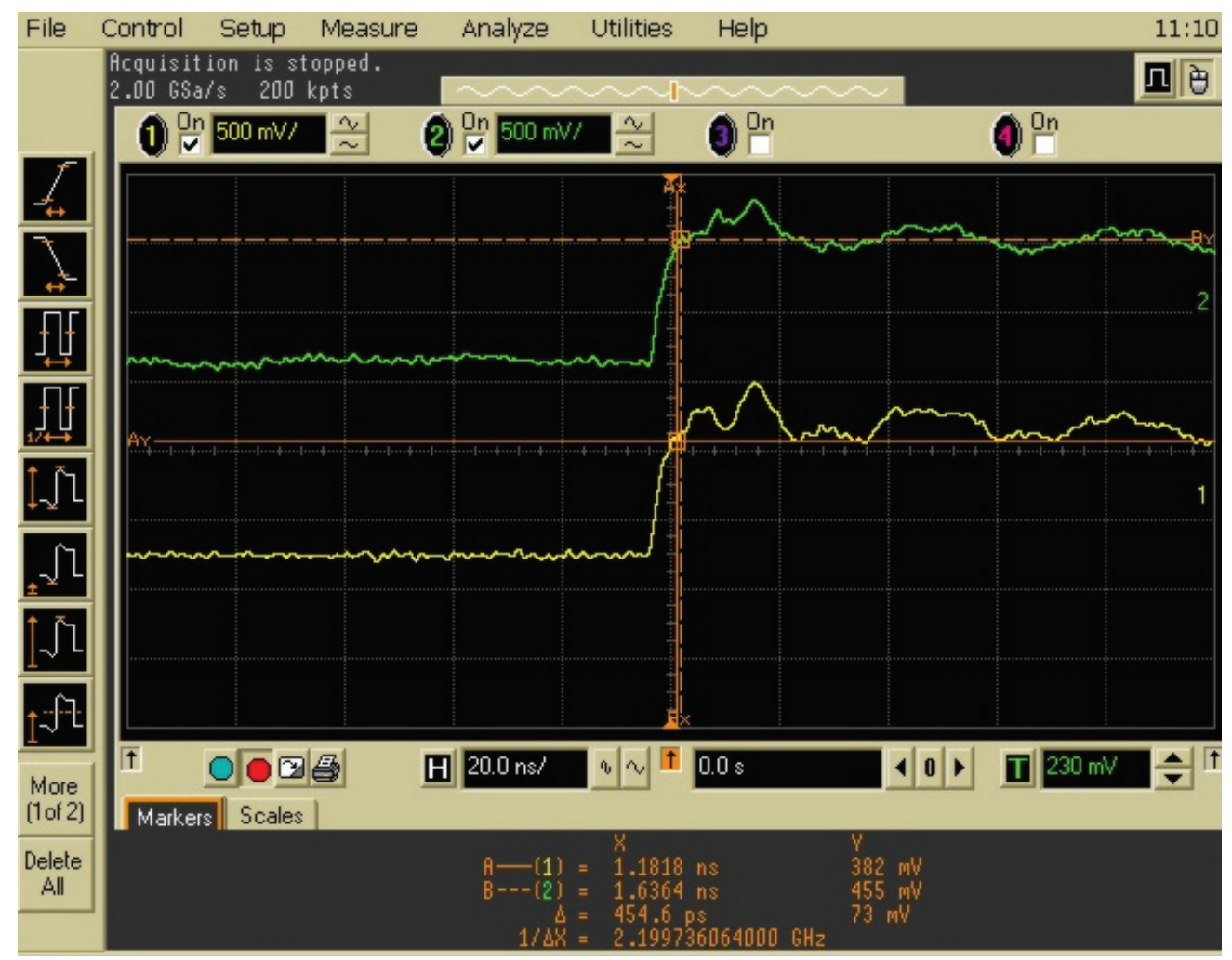

Figure 6.4: Time Difference Signal

Six ICs were tested and results documented. The following failures were witnessed and are discussed in the next section. 


\subsection{Analysis of Failed DUT}

The Board 1 was tested with the following parameters. The test bench used the Keithley 6220 precision current source, set to $200 \mu \mathrm{A}$, complaint voltage to $1.2 \mathrm{~V}$. Keithley power supply 2602 dual supply current supplying $1.2 \mathrm{~V}$ for power rails and $0.7 \mathrm{~V}$ for bias setting. The following table gives the voltage readings for the different rails after power up.

\begin{tabular}{|c||c|c|}
\hline Voltage rail & Value & Units \\
\hline VDD & 1.2 & $\mathrm{~V}$ \\
\hline Vbias & 700 & $\mathrm{mV}$ \\
\hline Iref & 200 & $\mu \mathrm{A}$ \\
\hline Voltage at Iref pin & 280 & $\mathrm{mV}$ \\
\hline
\end{tabular}

Table 6.1: Board 1 power up tests with DUT populated

Table 6.1 show that the DC bias is setup, and no shorts in the DUT.

The static Inputs are applied to check whether the TDA chains work. The below table shares the readings. Complimentary inputs are applied to the inputs shown in table and the outputs are measured. The board 1 showed a failure where the inputs to the differential pair were showing a short, upon further investigation using the multimeter it was found that the internal ESD diodes, referenced to the signal ground were conducting both ways, which shorted the inputs to signal ground. 


\begin{tabular}{|c||c|c|}
\hline Input Pin & Value & Units \\
\hline $\mathrm{In}$ & 1.2 & $\mathrm{~V}$ \\
\hline $\mathrm{InB}$ & 0 & $\mathrm{~V}$ \\
\hline $\mathrm{In} 1$ & 1.2 & $\mathrm{~V}$ \\
\hline In1B & 0 & $\mathrm{~V}$ \\
\hline
\end{tabular}

Table 6.2: Board 1 static input tests with DUT populated

The second DUT was also tested and after power ON, the outputs of the differential pair were shorting to ground, the bias values were correct as per Table 6.1. From this, behaviour the output ESD protection diodes were checked and were found to be conducting either way. Note that internal ESD protections were added to the input and output signal lines.

The third device under test were wirebonded with precautions taken to avoid ESD. The device was tested and it was found that it was working according to the static inputs applied. The output of one chain toggled when the input were toggled. This proves that both delay chains work due to the cross coupled architecture as discussed in chapter 4 . Later it was found that the outputs either clamped to VDD or signal ground. VDD value was scaled down to $800 \mathrm{mV}$.

\begin{tabular}{|c||c|c|}
\hline Voltage rail & Value & Units \\
\hline VDD & 800 & $\mathrm{mV}$ \\
\hline Vbias & 530 & $\mathrm{mV}$ \\
\hline Iref & 200 & $\mu A$ \\
\hline Voltage at Iref pin & 280 & $\mathrm{mV}$ \\
\hline
\end{tabular}

Table 6.3: DUT3 power up tests with DUT populated 


\begin{tabular}{|c|c|c|c||c|c|c|c||c|}
\hline In & InB & In1 & In1B & Out & OutB & Out1 & Out1B & Unit \\
\hline 0 & 0 & 0 & 0 & 0 & 0 & 600 & 0 & $\mathrm{mV}$ \\
\hline 0 & 800 & 0 & 800 & 0 & 0 & 600 & 0 & $\mathrm{mV}$ \\
\hline 800 & 0 & 800 & 0 & 0 & 800 & 600 & 0 & $\mathrm{mV}$ \\
\hline 800 & 800 & 800 & 800 & 0 & 800 & 600 & 0 & $\mathrm{mV}$ \\
\hline
\end{tabular}

Table 6.4: DUT 3 test result

Logic ' 1 ' is $800 \mathrm{mV}$ and logic ' 0 ' is $0 \mathrm{~V}$, so a combination of these signals were applied and one can notice that the signal "OutB" toggled as the inputs toggled, indicating that the TDA chains working. Due to the cross coupled architectures for the output to switch, both chains have to work. One can also notice that the signal "Out1" is at a constant of $600 \mathrm{mV}$, indicating a ESD diode failure. To confirm this hypothesis, the bias circuitry was turned off which gates all the pull down transistors, disconnecting the output to GND. Probing was done and the signals Out and Out1B was at GND potential, while the others were at VDD.

The reason DUT 3 worked for a short time was due to extra care taken during wirebonding. The wire used for wirebonding and the whole station are checked and it is found to be ESD safe. 


\begin{tabular}{|c|c|c|c||c|c|c|c||c|}
\hline In & InB & In1 & In1B & Out & OutB & Out1 & Out1B & Unit \\
\hline 0 & 800 & 0 & 800 & 0 & 800 & 0 & 800 & $\mathrm{mV}$ \\
\hline 800 & 0 & 800 & 0 & 800 & 0 & 800 & 0 & $\mathrm{mV}$ \\
\hline
\end{tabular}

Table 6.5: DUT 4 test result

The Table 6.5 shows another case where the TDA chains worked for a while and later failed. The device output toggled as the inputs toggled, both TDA chains were working, the failure occured later. The outputs were later clamped to VDD, irrespective of the input signal. To further test the scenario, the Iref and Vbias were disabled to isolate if the problem came from the circuit. VDD was powered on. The outputs were probed and found to be at VDD. The VDD was disabled, and then the IREF and Vbias were enabled, the outputs were showing a logic low. When all the power supplies were turned on the outputs clamped to VDD, irrespective of the input signal. This test explains the ESD diodes clamping the outputs to the power rails.

To eliminate failure of ESD diodes due to bonding, probing on the bare die was done. The probe station setup is shown in figure 6.5. Needle probes were used to land the connections to the bondpads. 


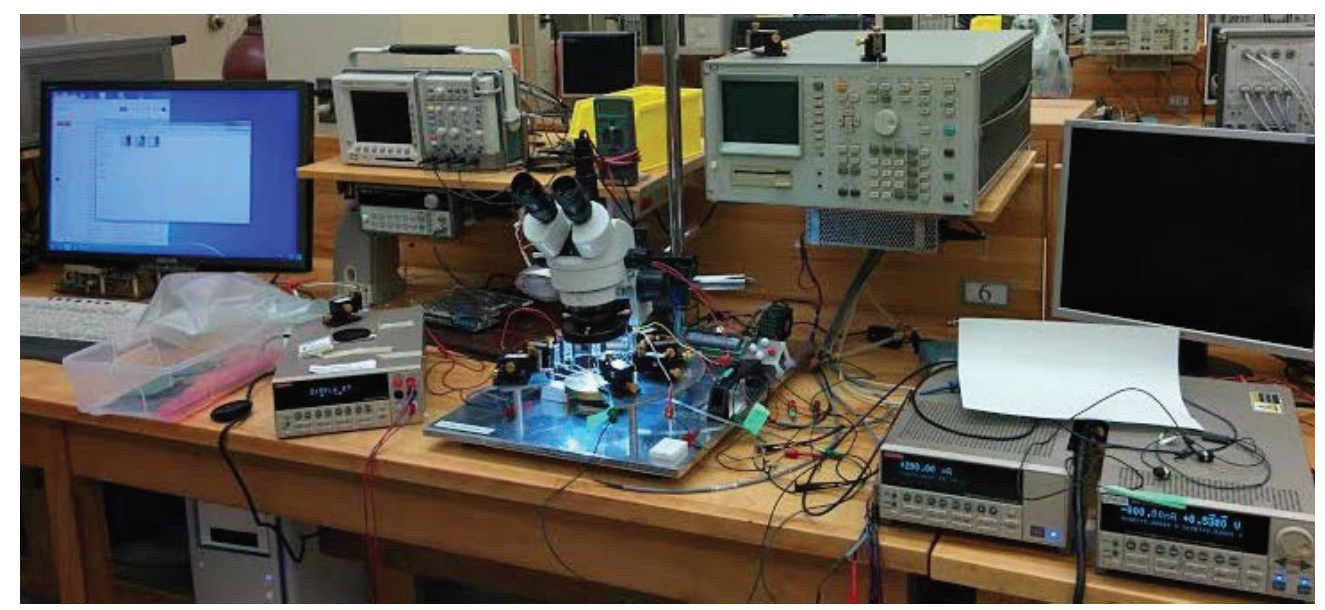

Figure 6.5: Probing station is setup with needle probes and connected to DC supply to test the chip. This is is done to eliminate any ESD effects on bonding.

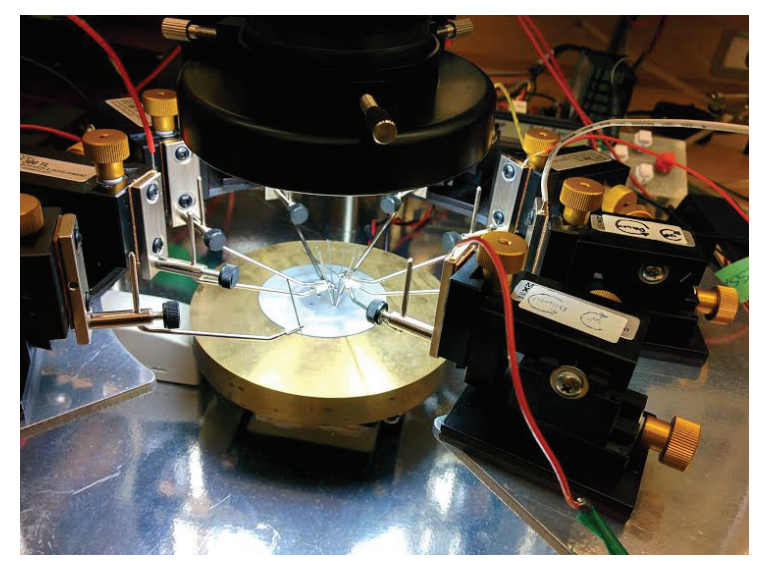

Figure 6.6: A closer view at the probe station revealing the connections to the needle probes.

Probing was done and was found that there were no issues with the wirebonding as the device functioned for a while, the outputs toggled. Upon further testing the IC's output was clamped to the VDD rail which was similar to the symptoms exhibited by the previous ICs. 


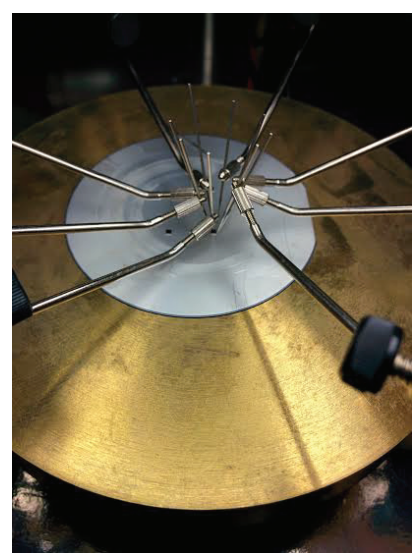

Figure 6.7: Die is epoxed to a 2 inch diameter wafer, 6 dies were epoxied for the probing purpose.

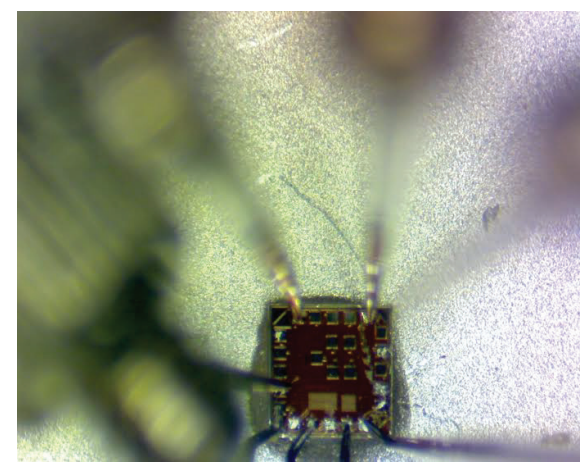

Figure 6.8: Photograph of the die with tungsten probes

At the end of these tests it is evident that the toggling of the pins, give an idea that both delay chains are working, as they are cross coupled. The failure occurs after a while where the outputs are clamped to the supply or ground ,indicating an ESD failure. Lowering of the supply voltage was also done to rule out the possibility of an ESD failure, but it remained the same. Experiments such as probing has ruled out the possibility of failure occuring during wirebonding, which leaves the fact that the failure occured at the manufactuing stage. 
This die area is shared with another design from university of Massachusetts, and the design does not contain any internal ESD diode protection and hence was probed to see the issue.

The outputs toggled and was consistent with the input combinations, proving the fact that this batch had manufacturing problems with internal ESD diode protection. The diode being weak and breaking down, clamping the outputs to either VDD or GND.

\begin{tabular}{|c|c|c|c|}
\hline $\mathrm{A}$ & $\mathrm{B}$ & Out & Unit \\
\hline 0 & 0 & 850 & $\mathrm{mV}$ \\
\hline 0 & 1200 & 1200 & $\mathrm{mV}$ \\
\hline 1200 & 0 & 850 & $\mathrm{mV}$ \\
\hline 1200 & 1200 & 1200 & $\mathrm{mV}$ \\
\hline
\end{tabular}

Table 6.6: Results from the circuit without ESD protection with $V D D=1.2 \mathrm{~V}$

\begin{tabular}{|c|c|c|c|}
\hline $\mathrm{A}$ & $\mathrm{B}$ & Out & Unit \\
\hline 0 & 0 & 500 & $\mathrm{mV}$ \\
\hline 0 & 800 & 800 & $\mathrm{mV}$ \\
\hline 800 & 0 & 500 & $\mathrm{mV}$ \\
\hline 1200 & 1200 & 800 & $\mathrm{mV}$ \\
\hline
\end{tabular}

Table 6.7: Results from the circuit without ESD protection with $V D D=800 \mathrm{mV}$ 


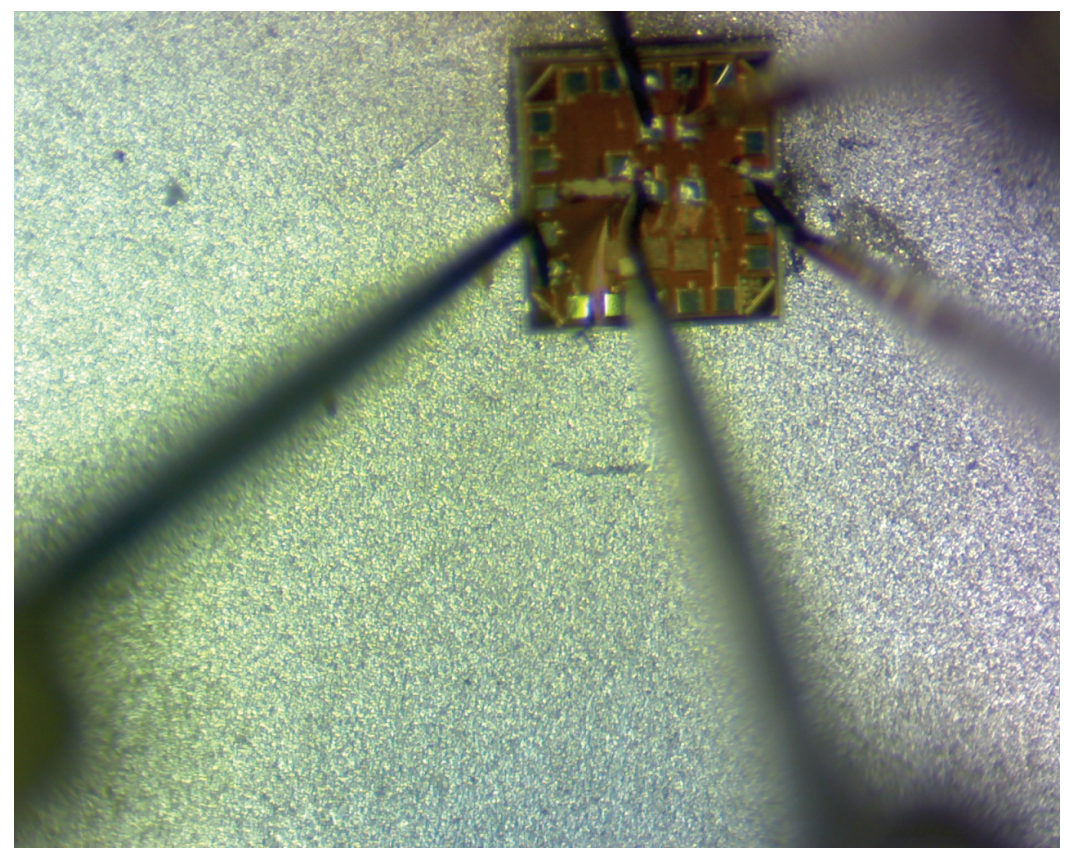

Figure 6.9: Photograph of the die with tungsten probes. Circuit without the ESD protection. 


\section{Chapter 7}

\section{Conclusion and future work}

The new approach to solve the linearity problem in vernier based time difference amplifier was proved to be successful. By lowering the gain it was easy to achieve high input range and minimum error in gain. It was also seen that the variations in the gain were minimal due to differential signalling schemes, and proved to be better than the single ended scheme. However the performance of the TDA on silicon could not be proven due to failure of the internal ESD diodes. The

new architecture meets the gain $2.4 \frac{\mathrm{p} s}{\mathrm{p} s}$, having an input range of $10 \mathrm{pS}$ to $1 \mathrm{nS}$, having a power consumption of less than $4 \mathrm{~mW}$. However the drawback in this design is that the amplifier is an open loop type, and has no feedback. Thus susceptible to PVT variations contributing to instability in the gain. The design of the architecture has a feature that can overcome the limitation, as there is an input to tune and correct the variations due to PVT by connecting a DLL circuit. The DLL circuit can also be used for tuning purpose to achieve a bigger gain for a minimum input range. Further work can be implemented by making the system closed loop in nature by adding a feedback mechanism such as a delay locked loop, 
to compensate for temperature and voltage variations.

This research is to build an experiment setup which is part of a bigger research and the specifications were given to meet the design of the test setup, however the rational behind the test setup was not known as no further information was provided. Hence further enhancements using other architectures can also be implemented to integrate to this experiment setup. 


\section{Bibliography}

[1] P. Dudek, S. Szczepanski, and J. Hatfield, "A high-resolution cmos time-todigital converter utilizing a vernier delay line," Solid-State Circuits, IEEE Journal of, vol. 35, pp. 240-247, Feb 2000.

[2] L.-S. Kim and R. Dutton, "Metastability of cmos latch/flip-flop," Solid-State Circuits, IEEE Journal of, vol. 25, pp. 942-951, Aug 1990.

[3] V. Stojanovic, V. Oklobdzija, and R. Bajwa, "A unified approach in the analysis of latches and flip-flops for low-power systems," in Low Power Electronics and Design, 1998. Proceedings. 1998 International Symposium on, pp. 227232, Aug 1998.

[4] M. Usama and T. Kwasniewski, "Metastability analysis of cmos current mode logic latches," in Electrical and Computer Engineering, 2005. Canadian Conference on, pp. 1521-1524, May 2005.

[5] T. Chaney and C. Molnar, "Anomalous behavior of synchronizer and arbiter circuits," Computers, IEEE Transactions on, vol. C-22, pp. 421-422, April 1973.

[6] C. Yan and M. Greenstreet, "Verifying an arbiter circuit," in Formal Methods in Computer-Aided Design, 2008. FMCAD '08, pp. 1-9, Nov 2008. 
[7] T. Yamaguchi, S. Komatsu, M. Abbas, K. Asada, N. N. Mai-Khanh, and J. Tandon, "A cmos flash tdc with 0.842013 ; 1.3 ps resolution using standard cells," in Radio Frequency Integrated Circuits Symposium (RFIC), 2012 IEEE, pp. 527-530, June 2012.

[8] V. Gutnik and A. Chandrakasan, "On-chip picosecond time measurement," in VLSI Circuits, 2000. Digest of Technical Papers. 2000 Symposium on, pp. 52-53, June 2000.

[9] M. Sorkhabi and S. Toofan, "A high resolution, multi-path gated ring oscillator based vernier time-to-digital converter," in Semiconductor Conference Dresden (SCD), 2011, pp. 1-4, Sept 2011.

[10] D. A. Bell, Fundamentals of electronic devices and circuits. Oxford University Press, Inc., 2009.

[11] Micrel, Programmable delay with fine tune control, July 2006.

[12] E. Raisanen-Ruotsalainen, T. Rahkonen, and J. Kostamovaara, "A lowpower cmos time-to-digital converter," Solid-State Circuits, IEEE Journal of, vol. 30, pp. 984-990, Sep 1995.

[13] P. Chen, S.-I. Liu, and J. Wu, "A cmos pulse-shrinking delay element for time interval measurement," Circuits and Systems II: Analog and Digital Signal Processing, IEEE Transactions on, vol. 47, pp. 954-958, Sep 2000.

[14] R. Staszewski, S. Vemulapalli, P. Vallur, J. Wallberg, and P. Balsara, "1.3 v 20 ps time-to-digital converter for frequency synthesis in 90-nm cmos," Circuits 
and Systems II: Express Briefs, IEEE Transactions on, vol. 53, pp. 220-224, March 2006.

[15] M. Lee and A. Abidi, "A 9 b, 1.25 ps resolution coarse 2013;fine time-todigital converter in $90 \mathrm{~nm}$ cmos that amplifies a time residue," Solid-State Circuits, IEEE Journal of, vol. 43, pp. 769-777, April 2008.

[16] R. Rashidzadeh, R. Muscedere, M. Ahmadi, and W. Miller, "A delay generation technique for narrow time interval measurement," Instrumentation and Measurement, IEEE Transactions on, vol. 58, pp. 2245-2252, July 2009.

[17] S. Mandai, T. Nakura, M. Ikeda, and K. Asada, "A 8bit two stage timeto-digital converter using 16x cascaded time difference amplifier in $0.18 \mathrm{um}$ cmos," in MELECON 2010 - 2010 15th IEEE Mediterranean Electrotechnical Conference, pp. 280-285, April 2010.

[18] S. Mandai, T. Iizuka, T. Nakura, M. Ikeda, and K. Asada, "Time-to-digital converter based on time difference amplifier with non-linearity calibration," in ESSCIRC, 2010 Proceedings of the, pp. 266-269, Sept 2010.

[19] M. Zanuso, S. Levantino, A. Puggelli, C. Samori, and A. Lacaita, "Time-todigital converter with 3-ps resolution and digital linearization algorithm," in ESSCIRC, 2010 Proceedings of the, pp. 262-265, Sept 2010.

[20] H.-J. Kwon, J. seung Lee, J.-Y. Sim, and H.-J. Park, "A high-gain wideinput-range time amplifier with an open-loop architecture and a gain equal to current bias ratio," in Solid State Circuits Conference (A-SSCC), 2011 IEEE Asian, pp. 325-328, Nov 2011. 
[21] P. Chen, S.-I. Liu, and J. Wu, "A low power high accuracy cmos time-todigital converter," in Circuits and Systems, 199\%. ISCAS '97., Proceedings of 1997 IEEE International Symposium on, vol. 1, pp. 281-284 vol.1, Jun 1997.

[22] F. Baronti, L. Fanucci, D. Lunardini, R. Roncella, and R. Saletti, "A highresolution dll-based digital-to-time converter for dds applications," in Frequency Control Symposium and PDA Exhibition, 2002. IEEE International, pp. 649-653, 2002.

[23] G. Nagaraj, S. Miller, B. Stengel, G. Cafaro, T. Gradishar, S. Olson, and R. Hekmann, "A self-calibrating sub-picosecond resolution digital-to-time converter," in Microwave Symposium, 200\%. IEEE/MTT-S International, pp. 2201-2204, June 2007.

[24] P. Chen, P.-Y. Chen, J.-S. Lai, and Y.-J. Chen, "Fpga vernier digital-to-time converter with 1.58 ps resolution and 59.3 minutes operation range," Circuits and Systems I: Regular Papers, IEEE Transactions on, vol. 57, pp. 1134-1142, June 2010 .

[25] S. Al-Ahdab, A. Mantyniemi, and J. Kostamovaara, "A 12-bit digital-totime converter (dtc) for time-to-digital converter (tdc) and other time domain signal processing applications," in NORCHIP, 2010, pp. 1-4, Nov 2010.

[26] B. Markovic, A. Tosi, F. Zappa, and S. Tisa, "Smart-pixel with spad detector and time-to-digital converter for time-correlated single photon counting," in IEEE Photonics Society, 2010 23rd Annual Meeting of the, pp. 181-182, Nov 2010. 
[27] Y. Cao, P. Leroux, W. De Cock, and M. Steyaert, "Design and assessment of a 6 ps-resolution time-to-digital converter with 5 mgy gamma-dose tolerance for nuclear instrumentation," in Advancements in Nuclear Instrumentation Measurement Methods and their Applications (ANIMMA), 2011 2nd International Conference on, pp. 1-8, June 2011.

[28] C. Salthouse, R. Weissleder, and U. Mahmood, "Development of a time domain fluorimeter for fluorescent lifetime multiplexing analysis," Biomedical Circuits and Systems, IEEE Transactions on, vol. 2, pp. 204-211, Sept 2008.

[29] A. Shanmugam and C. Salthouse, "3d lensless fluorescence imaging," in Instrumentation and Measurement Technology Conference (I2MTC), 2012 IEEE International, pp. 1109-1112, May 2012.

[30] D. O'Connor, Time-correlated single photon counting. Academic Press, 1984.

[31] A. Chan and G. Roberts, "A deep sub-micron timing measurement circuit using a single-stage vernier delay line," in Custom Integrated Circuits Conference, 2002. Proceedings of the IEEE 2002, pp. 77-80, 2002.

[32] M. Oulmane and G. Roberts, "A cmos time amplifier for femto-second resolution timing measurement," in Circuits and Systems, 2004. ISCAS '04. Proceedings of the 2004 International Symposium on, vol. 1, pp. I-509-12 Vol.1, May 2004.

[33] D. Kinniment, O. Maevsky, A. Bystrov, G. Russell, and A. Yakovlev, "Onchip structures for timing measurement and test," in Asynchronous Circuits and Systems, 2002. Proceedings. Eighth International Symposium on, pp. 190197, April 2002. 
[34] Analog devices, Fundamentals of Direct Digital Synthesis (DDS), 2009.

[35] A. Device, "Data sheet ad5933," AD5934, 2011.

[36] A. M. Abas, A. Bystrov, D. Kinniment, O. Maevsky, G. Russell, and A. Yakovlev, "Time difference amplifier," Electronics Letters, vol. 38, pp. 1437-1438, Nov 2002.

[37] A. Alahmadi, G. Russell, and A. Yakovlev, "Time difference amplifier design with improved performance parameters," Electronics Letters, vol. 48, pp. 562563, May 2012.

[38] A. N. M. Alahmadi, G. Russell, and A. Yakovlev, "Reconfigurable time interval measurement circuit incorporating a programmable gain time difference amplifier," in Design and Diagnostics of Electronic Circuits Systems (DDECS), 2012 IEEE 15th International Symposium on, pp. 366-371, April 2012.

[39] M. A. Abas, G. Russell, and D. Kinniment, "Design of sub-10-picoseconds onchip time measurement circuit," in Design, Automation and Test in Europe Conference and Exhibition, 2004. Proceedings, vol. 2, pp. 804-809 Vol.2, Feb 2004.

[40] S. Kim and S. Cho, "A variation tolerent reconfigurable time difference amplifier," in SoC Design Conference (ISOCC), 2009 International, pp. 301-304, Nov 2009.

[41] R. J. Baker, CMOS: circuit design, layout, and simulation, vol. 18. WileyIEEE Press, 2011. 
[42] B. Razavi, Design of analog CMOS integrated circuits. Tata McGraw-Hill Education, 2002.

[43] T. Nakura, S. Mandai, M. Ikeda, and K. Asada, "Time difference amplifier using closed-loop gain control," in VLSI Circuits, 2009 Symposium on, pp. 208-209, June 2009.

[44] C.-H. Lin and M. Syrzycki, "Pico-second time interval amplification," in SoC Design Conference (ISOCC), 2010 International, pp. 201-204, Nov 2010.

[45] S. Mandai, T. Nakura, M. Ikeda, and K. Asada, "Cascaded time difference amplifier using differential logic delay cell," in SoC Design Conference (ISOCC), 2009 International, pp. 194-197, Nov 2009.

[46] S. Mandai, T. Nakura, M. Ikeda, and K. Asada, "Cascaded time difference amplifier using differential logic delay cell," in Design Automation Conference (ASP-DAC), 2010 15th Asia and South Pacific, pp. 355-356, Jan 2010.

[47] S. Milicevic and L. MacEachern, "Frequency dividers implementing custom cells with resistor tail bias," in Signals, Systems and Electronics, 200\%. ISSSE '07. International Symposium on, pp. 493-496, July 2007.

[48] M. Li, W. Huang, T. Kwasniewski, and S. Wang, "A 0.18 um cmos transceiver design for high-speed backplane data communications," in Circuits and Systems, 2005. ISCAS 2005. IEEE International Symposium on, pp. 1158-1161 Vol. 2, May 2005.

[49] K. Mitzner, Complete PCB design using OrCAD Capture and PCB editor. Newnes, 2009. 
[50] E. Bogatin, Signal integrity: simplified. Prentice Hall Professional, 2004.

[51] H. W. Johnson, M. Graham, et al., High-speed digital design: a handbook of black magic, vol. 1. Prentice Hall Upper Saddle River, NJ, 1993. 


\section{Appendix A}

\section{Tools Used and Design}

\section{Methodology}

\begin{tabular}{|c|c|c|c|}
\hline Tool Chain & Version & Tool Provider & Purpose \\
\hline Cadence Virtuoso & 6.0 & Cadence & Design Suite \\
\hline Spectre & & Cadence & Transistor level Simulation \\
\hline Calibre & & Mentor Graphics & DRC Checker \\
\hline Assura & & Cadence & DRC, LVS and QRC extraction \\
\hline
\end{tabular}

Table A.1: Tools Used

The device was implemented using hierarchical design. The device consist of several individual components like, inverters ,multiplexers, etc.. Symbols are created for individual components and are verified for proper functionality using the Spectre simulator. Meeting the required specifications of the components is an iterative process that consists of adjusting transistor sizes, bias, and simulation.

The device are now integrated, and simulation is performed on the entire device. The device includes models such as bondpads, ESD protection devices that are required to interface to the external world. The above steps are repeated until the device specifications are met. The Electrical rule check is perfomed and the 
netlist is generated automatically from the schematic by the tool and is ready for layout.

The layout of the individual components are made and is verified for manufacturablility of the PDK using the rules provided by the manufacturer(CMC). For this verification tools such as Calibre and Assura are used.

The Assura tool is used to verify whether the layout and the schematic match, in-terms of transistor parameters and connectivity.

Once the LVS is clean, parasitics are extracted using the Assura QRC tool, and the simulations are run again to see if the device still meets the specification.

The Die size is estimated based on the area taken up by the individual components, bondpads, and other supporting structures. The pitch of the bondpads also play a vital role in selecting the die size.

Once is die size is fixed, the device floorplanning is done to fix the location of the cells and the pads.

A full chip routing is then performed, followed by a chip level DRC and LVS. The GDS stream out is perfomed on the full chip and the output is provided to the manufacturer. The manufacturer accept the design if their version of the DRC is run successfully. 


\section{Appendix B}

\section{Test Board Schematics}

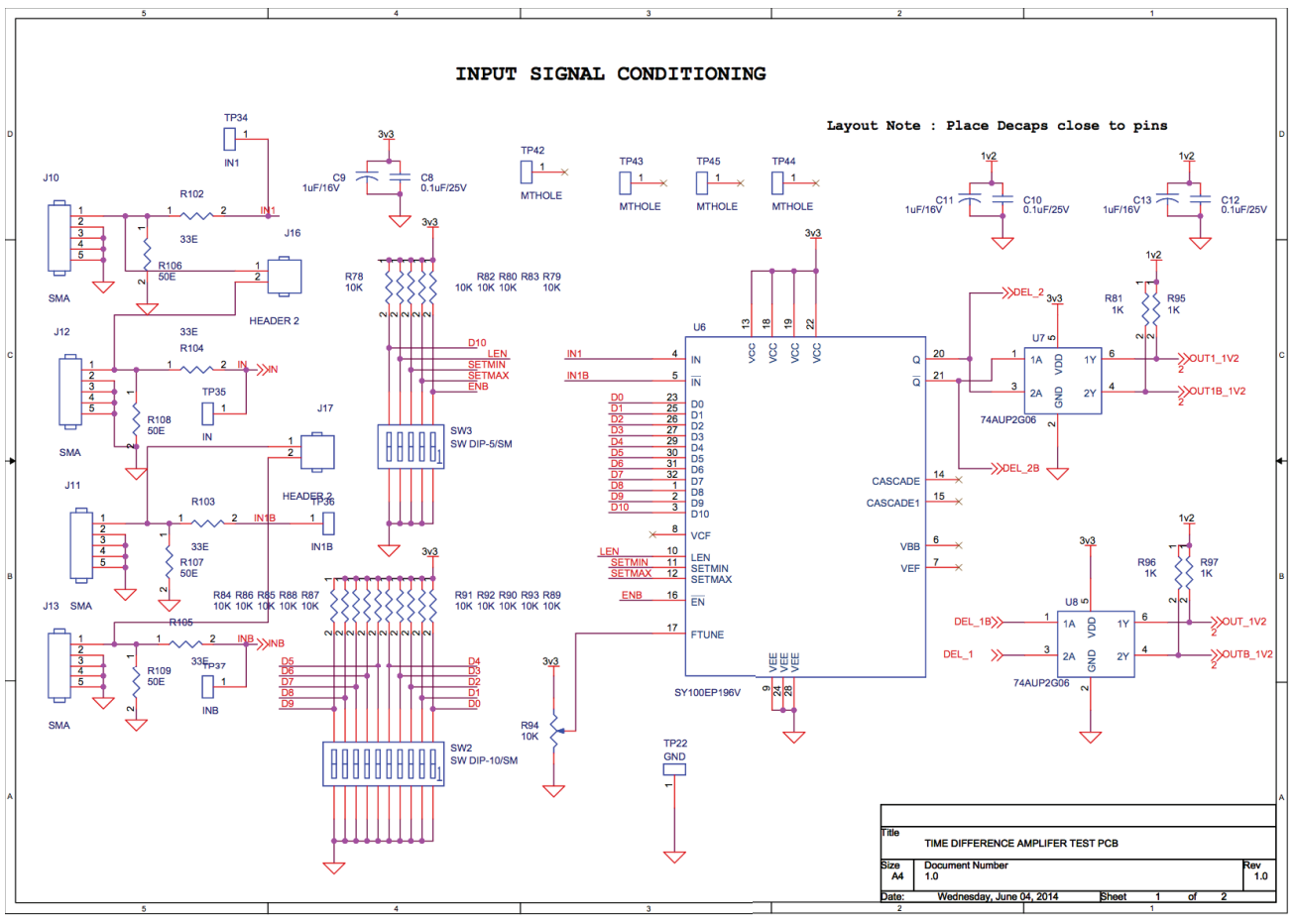

Figure B.1: PCB Schematics Page 1 


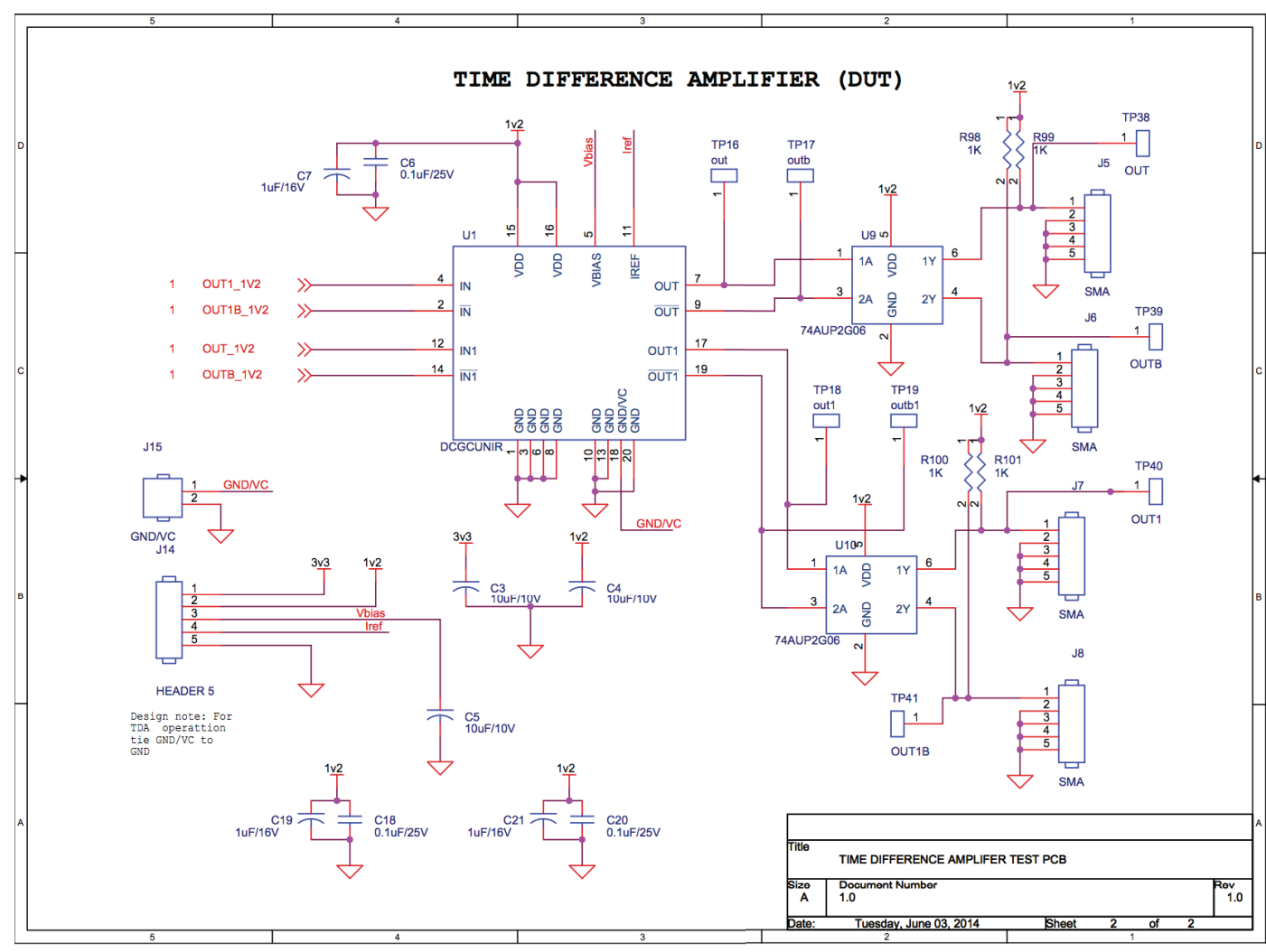

Figure B.2: PCB Schematics Page 2 


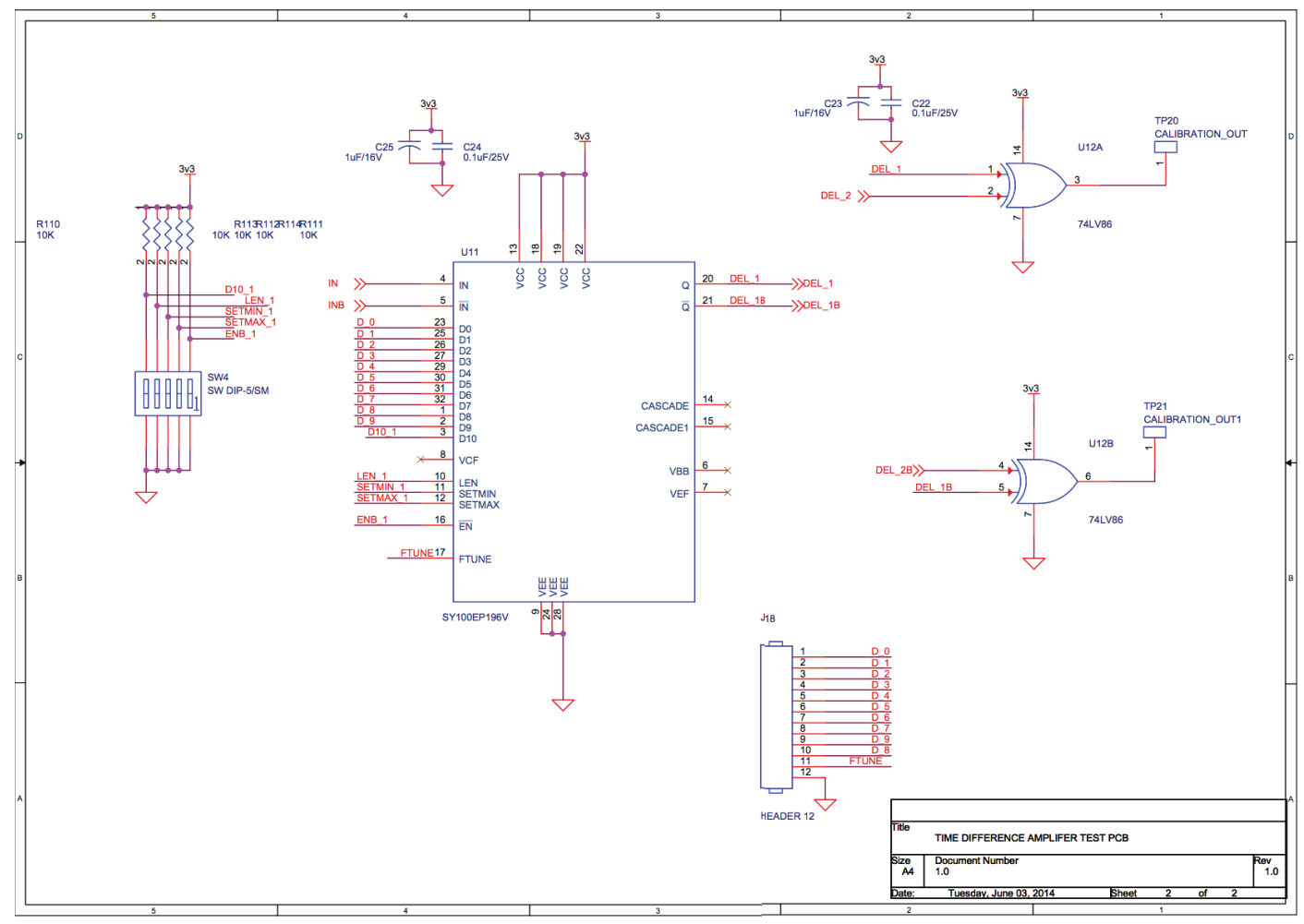

Figure B.3: PCB Schematics Page 3 


\section{Appendix C}

\section{Bill of Materials}

\begin{tabular}{|c|c|c|c|}
\hline Quantity & Manufacturer & Manufacturer Part Number & Description \\
\hline 3 & Kemet & T491A106M016AT & CAP TANT 10UF 16V 20\% 1206 \\
\hline 8 & Yageo & CC0603KRX7R8BB104 & CAP CER 0.1UF 25V 10\% X7R 0603 \\
\hline 8 & Kemet & T491A105K016AT & CAP TANT 1UF 16V 20\% 1206 \\
\hline 8 & TE Connectivity & $5-1814832-1$ & CONN SMA JACK STR 50 OHM PCB \\
\hline 28 & Panasonic & ERJ-2GEJ103X & RES 10K OHM 1/10W 5\% 0402 SMD \\
\hline 1 & Bourns Inc & 3361P-1-103GLF & TRIMMER 10K OHM 0.5W SMD \\
\hline 4 & Samsung & RC1608J330CS & RES 33 OHM 1/10W 5\% 0603 \\
\hline 4 & Yageo & RC0603FR-0749R9L & RES 49.9 OHM 1/10W 1\% 0603 SMD \\
\hline 1 & CTS & 210-10LPS & SWITCH SPST 10 SEC LOW PROF \\
\hline 2 & CTS & 210-5LPS & SWITCH SPST 5 SEC LOW PROF \\
\hline 1 & CMC & ICGCUNIR & TIME DIFFERENCE AMPLIFIER \\
\hline 2 & Micrel & SY100EP196VTG & IC DELAY LN 1024TAP PROG 32TQFP \\
\hline 4 & NXP Semiconductors & 74AUP2G06GW,125 & IC INVERTER DUAL LP 6TSSOP \\
\hline 1 & Texas Instruments & SN74LV86ADR & IC GATE XOR 4CH 2-INP 14-SOIC \\
\hline
\end{tabular}

Table C.1: Bill Of Materials 


\section{Appendix D}

\section{PCB Gerber}

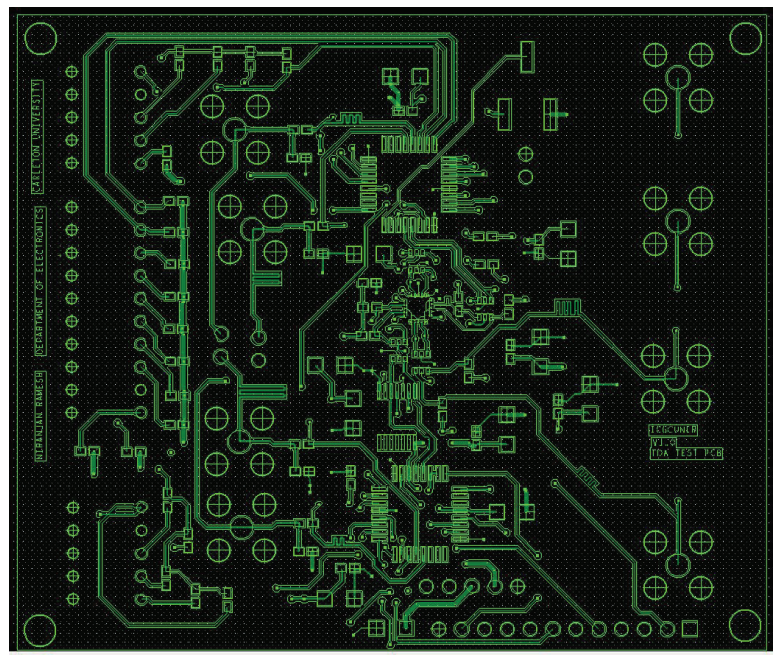

Figure D.1: Gerber : Top Layer 


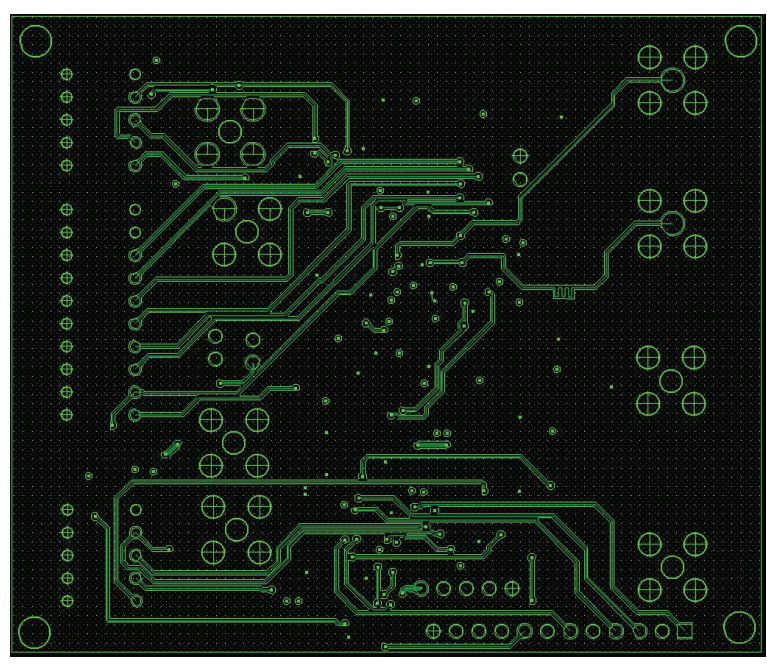

Figure D.2: Gerber : Bottom Layer

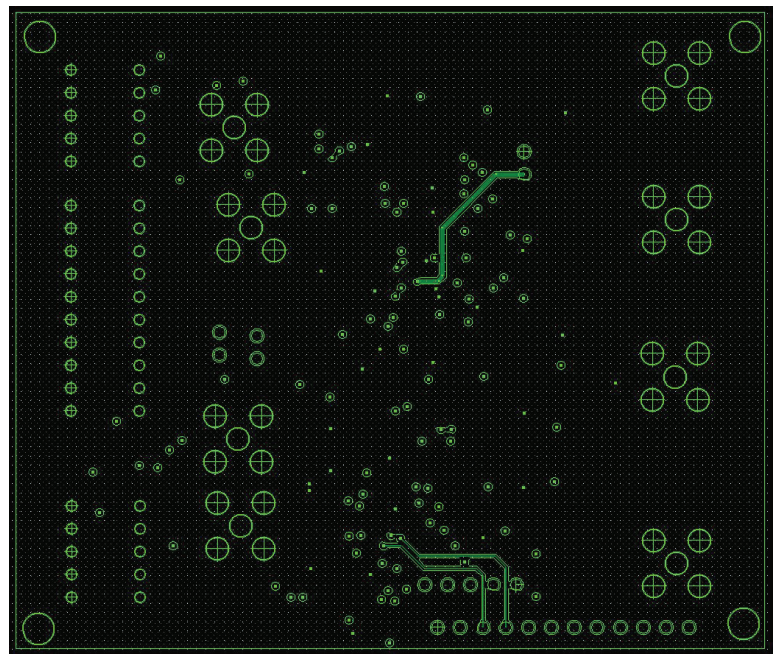

Figure D.3: Gerber : Ground Plane Layer 


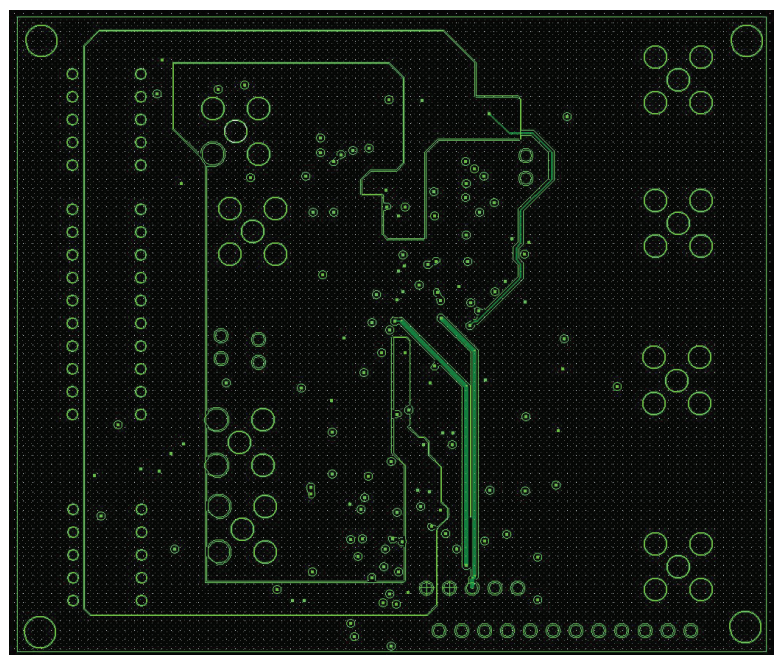

Figure D.4: Gerber : Power Plane Layer

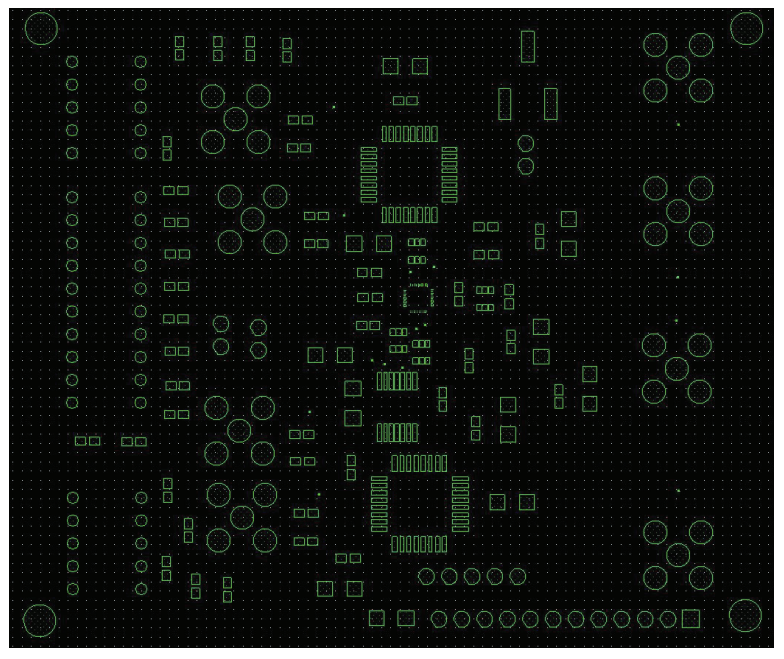

Figure D.5: Gerber : Solder mask top Layer 


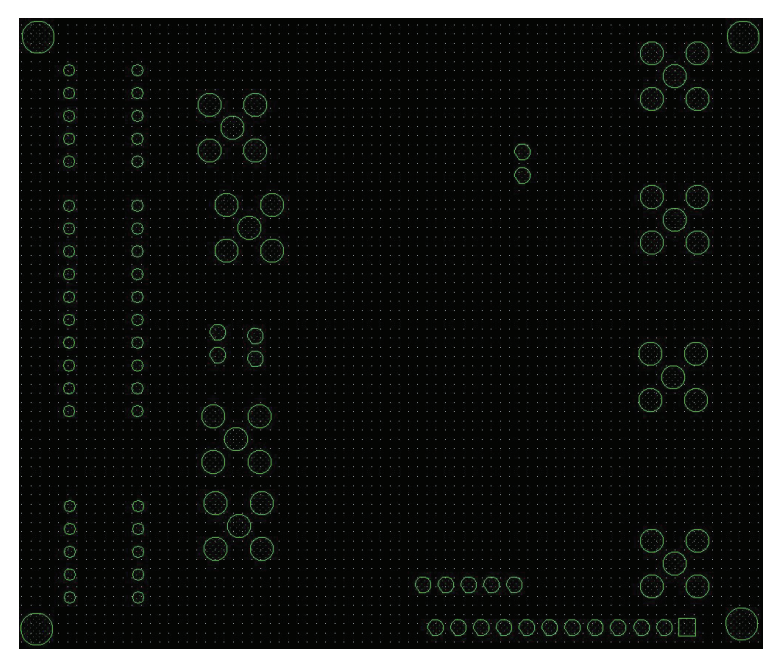

Figure D.6: Gerber : Solder mask Bottom Layer

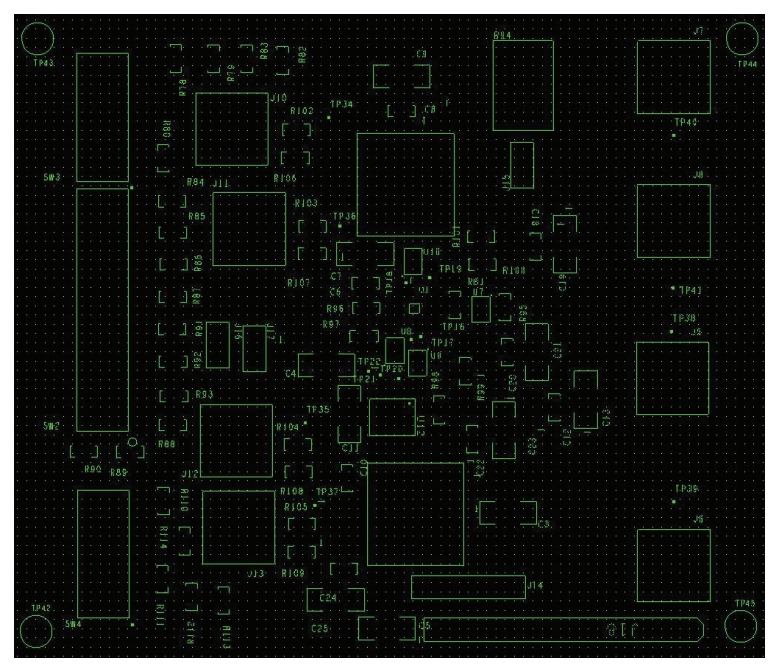

Figure D.7: Gerber : Silkscreen top Layer 